\title{
Stage-specific germ cell marker genes function in establishment and germ cell lineage commitment of pluripotent stem cells
}

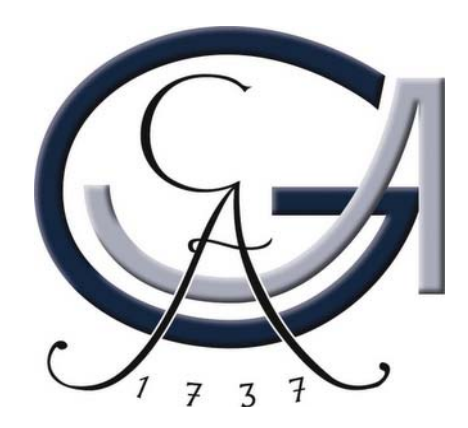

\section{DISSERTATION}

zur Erlangung des

Mathematisch-Naturwissenschaftlichen Doktorgrades

„Doctor rerum naturalium“

an der Georg-August-Universität Göttingen

\author{
vorgelegt von \\ Xingbo Xu \\ aus
}

Nei Mongol, China

Göttingen, 2012 
Referent: $\quad$ Prof. Dr. med. Dr. h. c. Wolfgang Engel

Korreferent: Prof. Dr. Sigrid Hoyer-Fender

Tag der mündlichen Prüfung: 20.09.2012 


\section{Dedicated to my family}

All I am, or can be, I owe to my supportive parents

There is no royal road to science, and only those who do not dread the fatiguing climb of its steep paths have a chance of gaining its luminous summits

- Karl Marx 


\section{Table of contents}

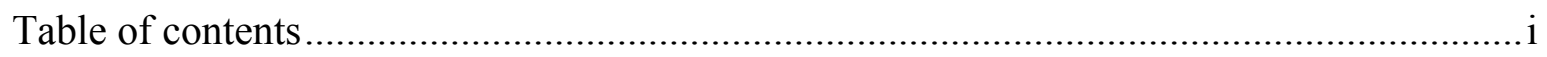

List of figures and tables ........................................................................................... ii

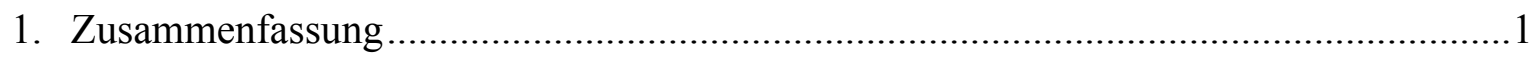

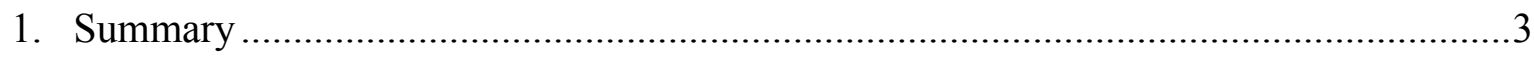

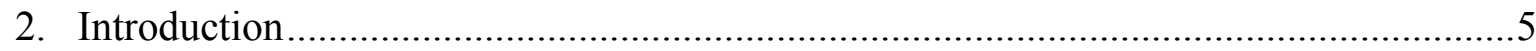

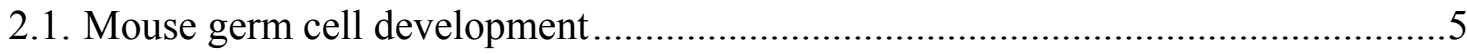

2.2. Developmental roles of selected stage-specific germ cell marker genes.................7

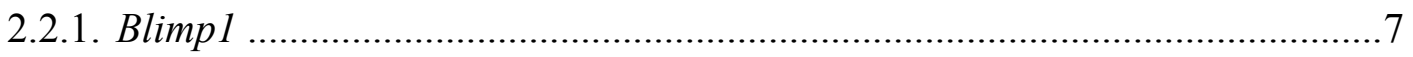

2.2.2. Ifitm 3 ............................................................................................ 7

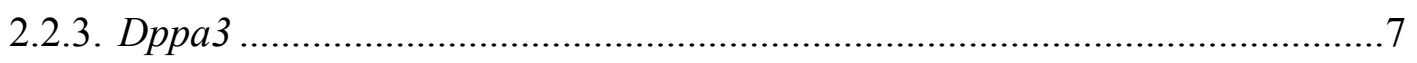

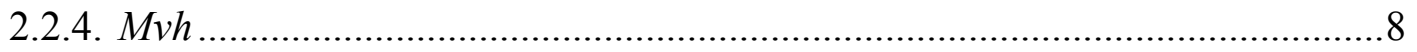

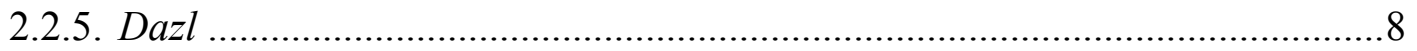

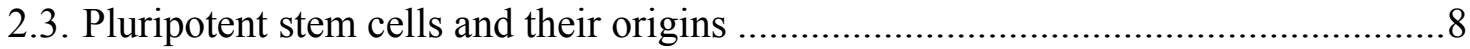

2.3.1. Induced pluripotent stem cells (iPSCs) ................................................. 10

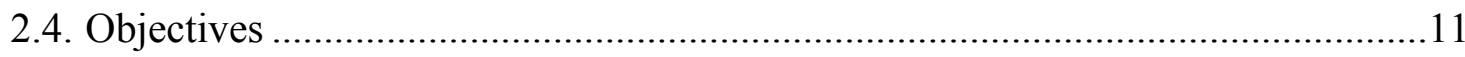

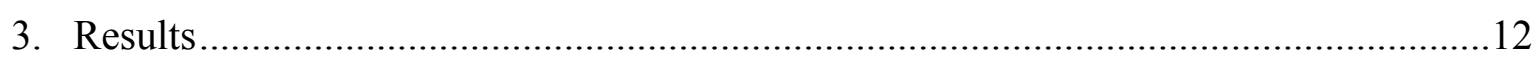

3.1. Stage-specific germ cell marker genes are expressed in all pluripotent cell types and emerges early during induced pluripotency ............................................. 13

3.2. Dppa3 binds to the IG-DMR of the Dlkl-Dio3 imprinting cluster and prevents

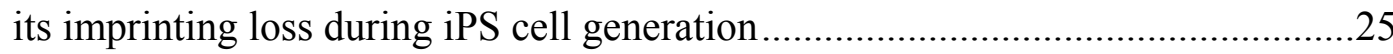

3.3. Mouse Dazl and its novel splice variant function in translational repression of

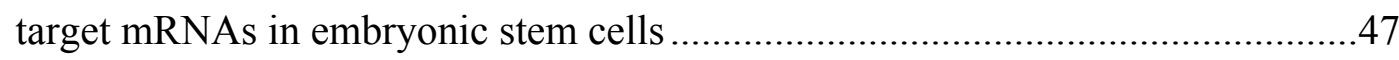

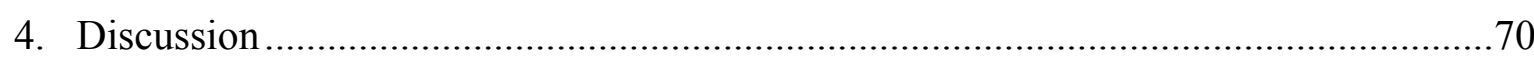

4.1. Spermatogenesis reconstituted from pluripotent stem cells in vitro......................70

4.2. Stage-specific germ cell marker genes are expressed in all mouse pluripotent cell types and emerge early during induced pluripotency ..................................72

4.2.1. Expansion of PGC fate model to human somatic cell reprogramming........73

4.2.2. Transdifferentiation of somatic cells into induced germline stem cells ......75

4.3. Dppa3 binds to the IG-DMR of the Dlk1-Dio3 imprinting cluster and prevents its imprinting loss during iPS cell generation.

4.3.1. Functional analysis of Dppa3 during somatic cell reprogramming by inducible transgenic mouse model 
4.4. Mouse Dazl and its novel splice variant functions in translational repression of target mRNAs in mouse embryonic stem cells................................................... 81

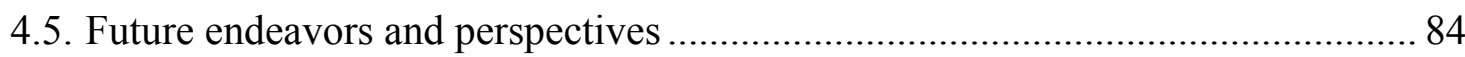

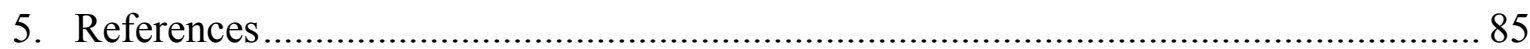

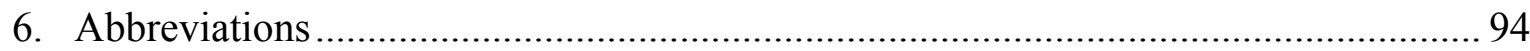

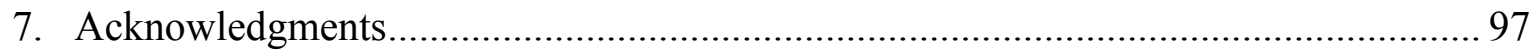

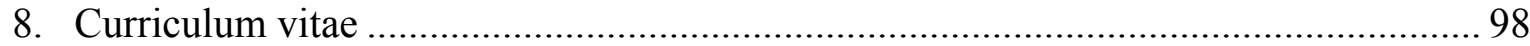

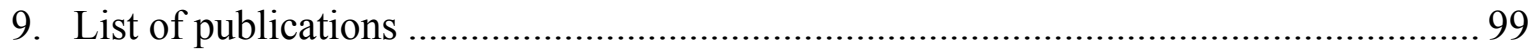

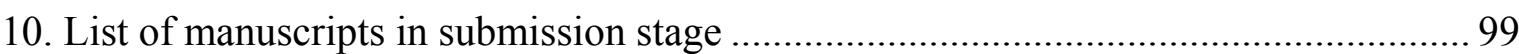




\section{List of figures and tables}

Figure 2.1. A schematic illustration of mouse primordial germ cell specification and migration during embryonic development.................................................. 6

Figure 2.2. Developmental origins of pluripotent stem cells ....................................... 9

Figure 2.3. The derivation and applications of iPS cells ........................................... 10

Figure 4.1. Expression pattern of GC/PrM- and pluripotency-marker genes during the course of hiPS generation ............................................................. 74

Figure 4.2. Schematic diagram depicting the generation of iGSCs .............................. 75

Figure 4.3. Generation and characterization of iGSC ................................................. 76

Figure 4.4. Characterization of iGSCs by immunocytochemistry ................................... 77

Figure 4.5. Schematic representation of Tet-on 3G system and Dppa3 transgenic mouse model generation ................................................................... 80

Figure 4.6. Schematic representation of induced Dppa3 expression during somatic

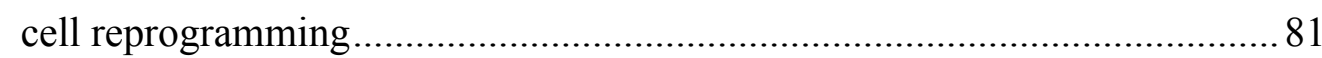

Figure 4.7. Dazl-Tat recombinant protein purification and functional test ...................... 82

Table 4.1 Pathological anomalies in human harbouring genetic defects at the Dlk1-Dio3 locus .79 


\section{Zusammenfassung}

Keimzellen transferieren genetische und epigenetische Informationen von Generation $\mathrm{zu}$ Generation. Ihre Eigenschaften haben Forscher angespornt, die molekularen Eigenschaften, die diese Besonderheit regulieren oder aufrechterhalten, zu untersuchen. Die jüngsten Entwicklungen haben gezeigt, dass eine Umwandlung von Stammzellen der Keimbahn zu pluripotenten Stammzellen möglich ist, was eine reziproke Beziehung dieser Zellen impliziert. Übereinstimmend mit diesen Ergebnissen haben mehrere Studien die Expression einiger Keimzellmarker (germ stem cell marker, GC marker) sowie prämeiotischer Marker (premeiotic marker, PrM marker) in verschiedenen pluripotenten Zelltypen aufgezeigt. Die Funktion und Relevanz der Expression dieser Markergene für die Etablierung sowie Erhaltung der Pluripotenz sind jedoch weitestgehend unbekannt. Im Rahmen dieser Studie sollte die Rolle von GC- und PrM Markergenen bei der Etablierung und Erhaltung sowohl der Pluripotenz als auch der Differenzierung aufgeklärt werden.

Im ersten Teil der vorliegenden Arbeit konnten wir zeigen, dass ausgewählte GCund PrM Markergene in allen analysierten pluripotenten Stammzellen exprimiert werden, was auf einen gemeinsamen Keimzellursprung von pluripotenten Stammzellen schließen lässt. Weiterhin werden GC Markergene vor der Aktivierung von Pluripotenzgenen während der Reprogrammierung somatischer Zellen zu induzierten, pluripotenten Zellen (iPS-Zellen) aktiviert. Zusammen mit den vorliegenden molekularen Beweisen für die in vivo Keimzellspezifizierung lassen diese Ergebnisse vermuten, dass während der frühen Phasen der Reprogrammierung somatischer Zellen eine temporäre Keimzellorientierung existiert.

Im zweiten Teil dieser Arbeit haben wir mittels Reprogrammierungsversuchen mit somatischen Zellen die Funktion ausgewählter GC Markergene bei der Etablierung und Erhaltung der authentischen Pluripotenz untersucht. Kürzlich wurde gezeigt, dass fehlerhaftes Imprinting am Dlk1-Dio3 Lokus hauptsächlich während der Etablierung von induzierten pluripotenten Stammzellen (iPSCs) auftritt. Interessanterweise wiesen alle iPS Zelllinien, die mit dem Dppa3 als ein GC Markergen generiert wurden, ein korrektes Imprintingmuster am Dlk1-Dio3 Lokus auf. Diese Ergebnisse lassen vermuten, dass GC Markergene, insbesonders Dppa3, wichtig für die Etablierung authentischer Pluripotenz sind. 
Im dritten Teil der vorliegenden Arbeit wurde die Rolle von Dazl als ein PrM Markergen in post-transktiptionellen Regulationsmechanismen sowohl in pluripotenten Stammzellen als auch in Keimzellen untersucht. Unsere Ergebnisse deuten daraufhin, dass Dazl die Keimzellspezifizierung befördert und möglicherweise als ein translationeller Repressor fungiert, der die Balance zwischen dem Beitrag zur Keimbahn und zur Pluripotenz aufrechterhält.

Zusammenfassend betonen die Ergebnisse unserer Studien die Bedeutung von GC Markergenen in der Etablierung der Pluripotenz in vivo und in vitro und bekräftigen die Hypothese eines Keimzellursprungs aller pluripotenten Stammzellen. Somit konnten unsere Arbeiten einen Beitrag zur Analyse der Rolle von GC und PrM Markergenen in pluripotenten Stammzellen geben. 


\section{Summary}

Germ cells are firmly committed to fulfil the mandate of transmitting genetic and epigenetic information from one generation to the next. Their mysterious characteristics have fascinated biological researchers to study the molecular mechanisms which regulate or maintain this speciality. The recent advances have shown the possibility of conversion between germline stem cells and pluripotent stem cells, implicating their reciprocal relationship. In line with these observations, several studies have shown the expression of some germ stem cell (GC) and premeiotic (PrM) marker genes in various pluripotent cell types. However, the function and relevance of this marker genes expression for pluripotency establishment and maintenance is largely unknown. The aim of this thesis was to elucidate the role of GC and PrM marker genes in establishment/maintenance of pluripotency as well as in differentiation.

In the first part of this thesis, we demonstrated the expression of selected GC and PrM marker genes in all analysed pluripotent stem cells, suggesting a common germ cell origin of pluripotent stem cells. Further, the GC marker genes were found to be activated prior to the activation of pluripotency-related genes during somatic cell reprogramming towards induced pluripotency. These results together with the available molecular evidence for in vivo germ cell specification led us to suggest a possible existence of a temporary germ cell fate during early stages of somatic cell reprogramming.

In the second part of this study, we examined the function of selected GC marker genes in establishment and maintenance of authentic pluripotency using somatic cell reprogramming studies. Recently, defects in imprinting at the Dlk-Dio3 locus were shown to occur mostly during establishment of induced pluripotent stem cells (iPSCs). Interestingly, all iPS cell lines generated in the presence of Dppa3, a GC marker gene, led to the proper imprinting maintenance at the Dlkl-Dio3 locus. These results suggest that Dppa3 is important for the establishment of authentic pluripotency.

In the third part of this thesis, a PrM marker gene, Dazl was studied for its role in post-transcriptional regulation mechanisms in pluripotent stem cells as well as in germ cells. Our results suggest that Dazl, a germ cell lineage mediator, might function as a translational repressor to maintain the balance between germ cell lineage commitment and pluripotency.

Collectively, the results of our studies emphasize the importance of GC marker genes in establishment of pluripotency in vivo and in vitro, and strengthen the hypothesis 
of germ cell origin of all pluripotent stem cells. Our studies shed light on the undiscovered role of GC/PrM marker genes in pluripotent cells. 


\section{Introduction}

"Germ cells are the most fascinating cells of all cell types and are still deeply mysterious" (McLaren, 1994). The biological significance of germ cells is to perpetuate the parental genetic information to the next generation. To this end, germ cells undergo extensive genetic and epigenetic reprogramming through complex biological processes such as chromatin condensation, meiosis and morphological transformation into mature gametes. They are specified during embryonic development and can be distinguished from other somatic cells by their unique gene expression profiles. Intriguingly, the expression of germ cell marker genes is not only restricted to germ cells, but are also highly expressed in pluripotent inner cell mass (ICM) and various pluripotent cell types. This may indicate that all pluripotent cells might have the same germ cell origin or probably the expression of germ cell marker genes is indispensable for establishment and maintenance of pluripotency. The goal of this thesis was to investigate the importance of these germ cell marker genes in pluripotent stem cells as well as during the induction of pluripotency in somatic cells. Hence, the role of selected germ cell marker genes during germ cell development will be highlighted along with a brief introduction to origins of pluripotent stem cells.

\subsection{Mouse germ cell development}

During mouse early embryogenesis, primordial germ cells (PGCs), the founder population of germ cells, are specified at proximal epiblast, undergo further development and finally localize to the genital ridge at midgestation (Ginsburg et al., 1990). After reaching puberty, germ cells are developed into mature haploid spermatozoa or oocytes. Then a new cycle will start again upon fertilization, representing the beginning of a new individual.

PGCs specification is induced by instructive signal, Bone Morphogenetic Protein (BMP), which is secreted from extra embryonic ectoderm prior to gastrulation at embryonic day E6.25. A small cluster of PGC precursors (approximately 8 cells) appear at the proximal epiblast at this stage (Ohinata et al., 2005). During this time, Blymphocyte-induced maturation protein-1 (Blimpl) and Interferon-induced transmembrane protein 3 (Ifitm3, also known as Fragilis), two typical early germ stem cell marker genes are necessarily expressed and function in differentiation suppression thereby maintaining the PGC congregation (Vincent et al., 2005; Tanaka et al., 2005). At 
E7.5, about 50 PGCs are completely separated in the extra- embryonic mesoderm at the base of the allantois (Ginsburg et al., 1990). These cells can be easily distinguished by the expression of Alkaline Phosphatase $(A P)$, a surface marker for germ cells as well as pluripotent stem cells, and Developmental pluripotency-associated protein 3 (Dppa3), another important germ stem cell marker gene (Saitou et al., 2002). From this region, PGCs migrate and proliferate while passing through primitive streak, embryonic endoderm to reach hind gut with around 250 cells at E9.5 (Tam and Snow 1981). Later on, PGCs change direction and move towards dorsal mesentery and colonize in the genital ridges at E12.5, where about 4000 PGCs start to undergo sex-specific differentiation to form mature gametes (De Felici et al., 1992). The pre-meiotic specific genes Mouse vasa homologue $(M v h)$ and Deleted in azoospermia-like (Dazl) start to express at E10.5 and E11.5, respectively. They are believed to have essential roles in fate determination event of the germ cell and in fundamental events of post-migratory germ cell development (Fujiwara et al., 1994; Haston et al., 2009). The process of germ cell specification and development is illustrated in figure 2.1.

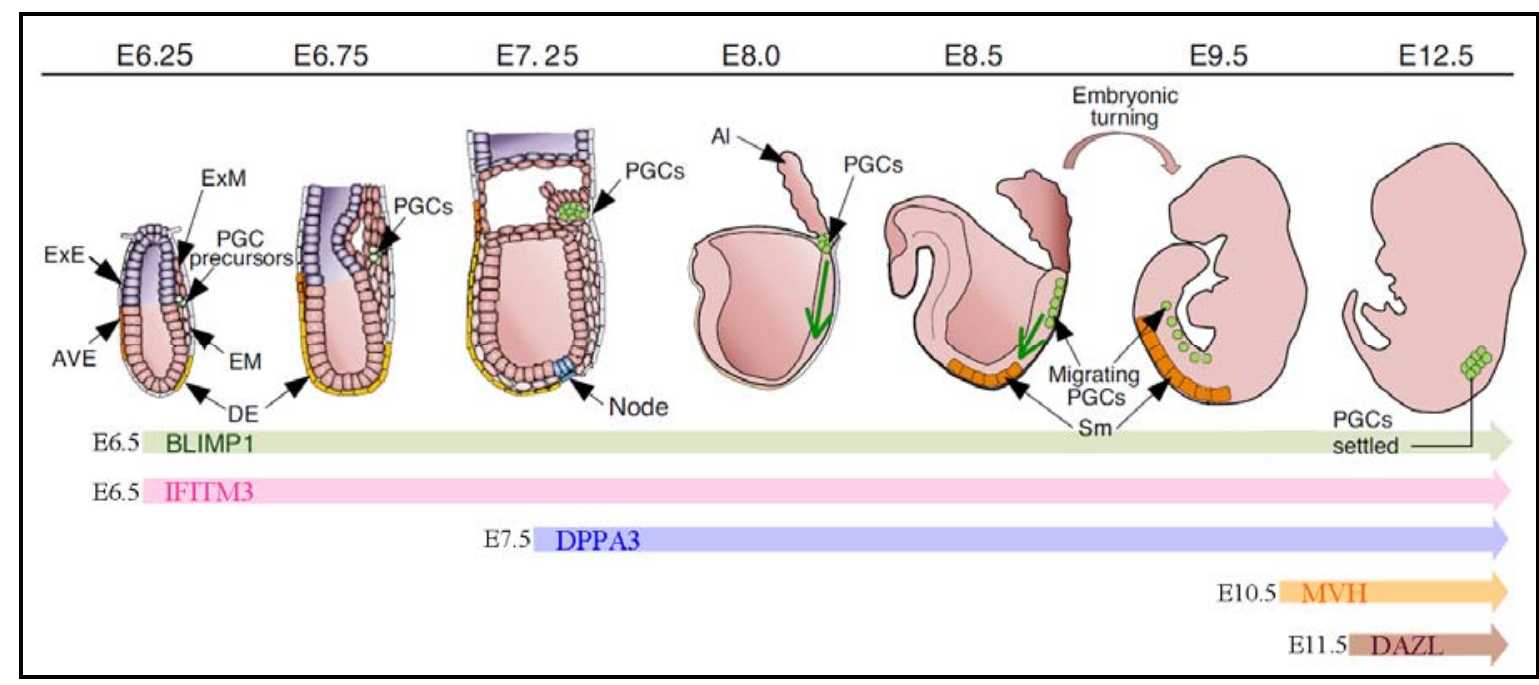

Figure 2.1. A schematic illustration of mouse primordial germ cell specification and migration during embryonic development. Primordial germ cell (PGC) precursors (E6.25) and PGCs are presented as green circles during embryonic development from E6.25 to E12.5, and the PGC migration route is indicated by a green arrow. The timing of germ cell specific marker genes Blimp1, Ifitm3, Dppa3, Mvh, and Dazl expression is shown below. Al, allantois; AVE, anterior visceral endoderm; DE, distal endoderm; DVE, distal visceral endoderm; EM, embryonic mesoderm; Epi, epiblast; ExE, Extra-embryonic ectoderm; ExM, extra-embryonic mesoderm; PGC, primordial germ cells; VE, visceral endoderm (Figure adapted and modified from: Saitou et al., 2012). 


\subsection{Developmental roles of selected stage-specific germ cell marker genes}

2.2.1. Blimp1 is expressed as early as in the founder PGCs at E6.25 and functions as a determinant of PGC specification (Ohinata et al., 2005). Blimpl encodes a transcriptional repressor protein which contains an N-terminally located positive regulatory domain functioning in chromatin remodeling; a proline-rich region responsible for the recruitment of transcriptional co-repressor and a C-terminally located five krüppel-type zinc finger motifs for DNA binding (Ren et al., 1999; Yu et al., 2000). Strikingly, Blimp1 knock-out studies showed a complete depletion of PGCs at E7.25 when germ cell specification initiates (Vincent et al., 2005). A recent study reported the successful establishment of embryonic stem cells (ESCs) and epiblast stem cells (EpiSCs) from Blimp1-deficient blastocyst and epiblast, respectively (Bao et al., 2012). These results suggest that Blimp1 expression is only obligatory for the PGCs specification but not for the establishment and maintenance of pluripotency.

2.2.2. Ifitm 3 is a member of interferon-induced transmembrane protein gene family. The encoded protein contains two transmembrane domains and localizes on the cell surface (Lange et al., 2003). Upon the BMP signaling stimulation, the expression of Ifitm3 is activated as early as in PGC precursors (Lange et al., 2003). It is believed that Ifitm3 expression is important for the congregation as well as PGCs migration towards the genital ridge (Tanaka et al., 2005; Saitou et al., 2002). Contrary to this hypothesis, genetic depletion of Ifitm3 does not result in any detectable effect on embryonic development, viability and fertility, possibly due to the existence of compensatory mechanisms (Lange et al., 2008).

2.2.3. Dppa3 was initially identified as a PGC marker gene expressed at E7.25 embryo, therefore it is also named as PGC7 (Sato et al., 2002). This gene encodes a protein containing both nuclear localization signal and nuclear export signal allowing it to shuttle between nucleus and cytoplasm (Saiti et al., 2007). Dppa3 is known to harbor a DNAbinding domain responsible for chromosomal organization at the N-terminus of the protein and a splicing factor like motif involved in RNA processing at the C-terminus (Aravind et al., 2000). Experimentally it was also proven that Dppa3 is able to bind both on DNA and RNA (Aravind et al., 2000). Dppa3 is inherited as a maternal factor since its expression is detected in oocytes and in zygotes, later on the inherited expression is replaced by the onset of zygotic expression, which persists until preimplantation embryo 
development (Payer et al., 2003). Dppa3 expression is silenced until E7.25, there after, is restricted and mark PGCs during migration till their colonization at genital ridges (Sato et al., 2002). Even though Dppa3 is used as a germ stem cell marker gene, it seems to be not important for PGC emergence (Bortvin et al., 2004; Payer et al., 2003). Dppa3 knockout studies showed that the first generation mutant mice have no significant defects in germ cells development and differentiation (Bortvin et al., 2004). However, the second generation mutant embryos die at early cleavage stages emphasizing the significance of Dppa3 as a maternal factor indispensible for pre-implantation embryo development (Bortvin et al., 2004).

2.2.4. $\boldsymbol{M v h}$ encodes an ATP-dependent RNA helicase of the DEAD-box protein family (Hay et al., 1990). In PGCs, the expression of Mvh is detectable after PGC colonized in the genital ridge at around E10.5. It is continuously expressed until the sex-specific germ cell differentiation to generate mature gametes (Toyooka et al., 2000). Furthermore, it is also believed that the $M v h$ expression is in response to a germ-soma interaction (Toyooka et al., 2000). Genetic ablation studies revealed that $M v h$-null male mice are sterile, while females are fertile (Tanaka et al., 2000).

2.2.5. Dazl codes for an RNA binding protein that is important for germ cell differentiation in vertebrates (Peng et al., 2009). Dazl protein contains an RNA recognition motif (RRM) that mediates the interaction with its target mRNAs, as well as a DAZ motif and a proline-rich region, which might be involved in protein-protein interactions (Tsui et al., 2000a). Dazl expression is first detectable in post-migratory PGCs at E11.5 and persists till gametogenesis (Cauffman et al., 2005). It is localized in both nucleus and cytoplasm of fetal gonocytes, but relocates to the cytoplasm during male meiosis (Reijo et al., 2000). The deletion of mouse Dazl leads to the loss of germ cells in both male and female gonads (Ruggiu et al., 1997). Additionally, it has also been reported that $\mathrm{Dazl}$ deficiency leads to a reduction in post-migratory PGCs number, failure to re-establish genomic imprints, and defects in meiotic progression (Haston et al., 2009).

\subsection{Pluripotent stem cells and their origins}

Pluripotent stem cells have the capacity of prolonged self-renewal and the ability to differentiate into all three germ layers (ectoderm, endoderm and mesoderm). However, alone they can not give rise to a fetus or entire organism because of lacking the ability of 
differentiating into extra-embryonic tissues such as placenta (Byrne, 2011). Based on the origin of the cells which are used for the establishment of pluripotent cells, the pluripotent stem cell lines can be currently defined as follows: [1] Embryonic Stem Cells (ESCs) derived from the Inner Cell Mass (ICM) of E3.5 day mouse blastocysts (Evans et al., 1987; Martin, 1981), [2] Epiblast Stem Cells (EpiSCs) derived by the culture of proximal region of E5.5-6.5 embryo epiblast (Brons et al., 2007; Tesar et al., 2007), [3] Embryonic Germ Cells (EGCs) derived from the in vitro culture of PGCs, which have colonized the genital ridge at E12.5 (Matsui et al., 1992; Resnick et al., 1992), [4] Embryonic Carcinoma Cells (ECCs) derived from either testicular or ovarian teratomas of embryos (Kleinsmith and Pierce 1964; Finch and Ephrussi 1967; Kahan and Ephrussi 1970), [5] multipotent Germ line Stem Cells (mGSCs) and multipotent adult Germ line Stem Cells (maGSCs) derived from neonatal and adult mouse testis, respectively (Kanatsu-shinohara et al., 2004, Guan et al., 2006), and [6] induced Pluripotent Stem Cells (iPSCs), generated by reprogramming of somatic cells by enforced expression of several ectopic transcriptional factors such as Oct4, Sox2, Klf4, c-Myc, Nanog, and Lin28 (Takahashi and Yamanaka 2006; Takahashi et al., 2007). The developmental origin of fore-mentioned pluripotent stem cells is depicted in figure 2.2.

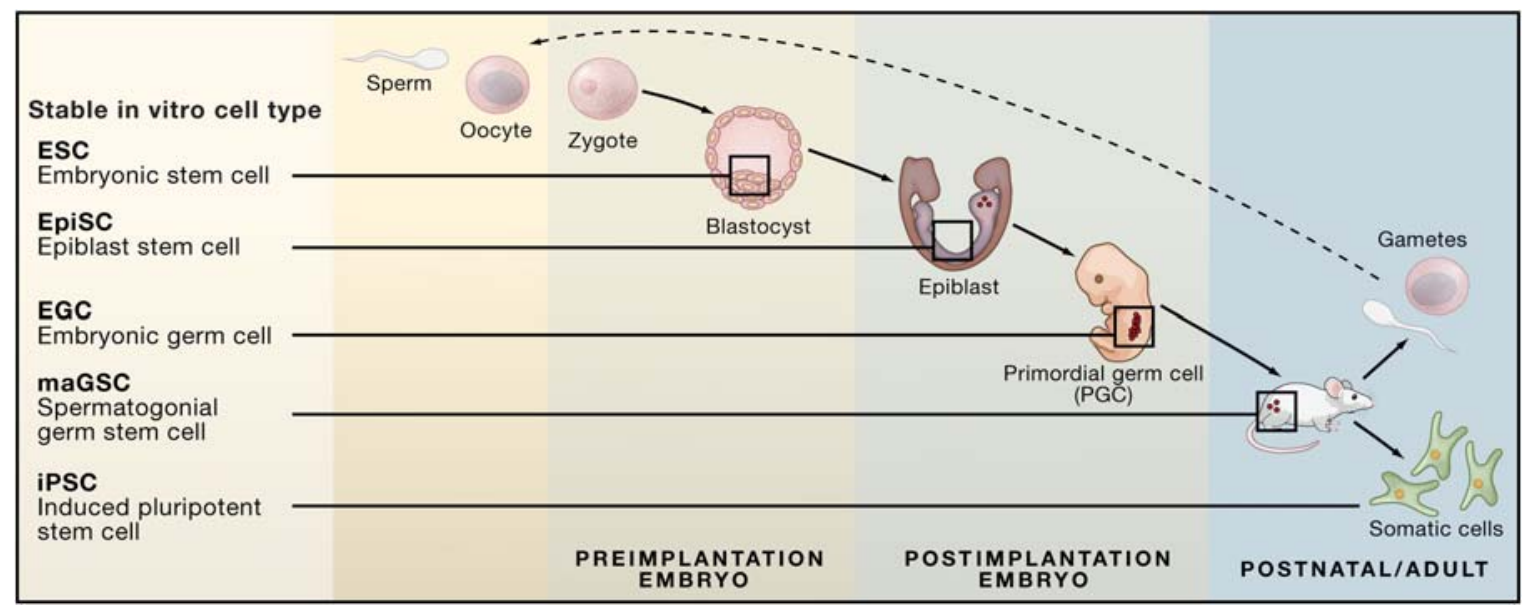

Figure 2.2. Developmental origins of pluripotent stem cells. Different types of pluripotent cells can be derived by explanting cells at various stages of mouse embryonic development or from neonatal and adult mouse testis. Induced pluripotent stem cells (iPSCs) are derived by direct reprogramming of somatic cells in vitro by forced expression of certain transcriptional factor cocktails. (Figure adapted from: Hanna et al., 2010).

Tracing back to the origin of above mentioned pluripotent cell lines highlights that EpiSCs, EGCs, ECCs, GSCs and maGSCs, all have the germ line origin, except ESCs and iPSCs whose origins or transitions are currently not clearly understood. Due to the 
developmental stage of isolation, they have their unique gene expression marks to distinguish from each other. However, they all share the expression of germ cell stage specific marker genes such as Blimp1, Ifitm3, Dppa3, Mvh and Dazl. This may indicate that all pluripotent cell types could have a germ cell origin or might transit through the germ cell fate and these marker genes are all necessary for maintenance of pluripotency.

\subsubsection{Induced pluripotent stem cells (iPSCs)}

iPSCs are being considered as the most promising tool which can be used in disease modeling, regenerative medicine applications, and drug screening because of their high similarities to ESCs (Fig. 2.3).

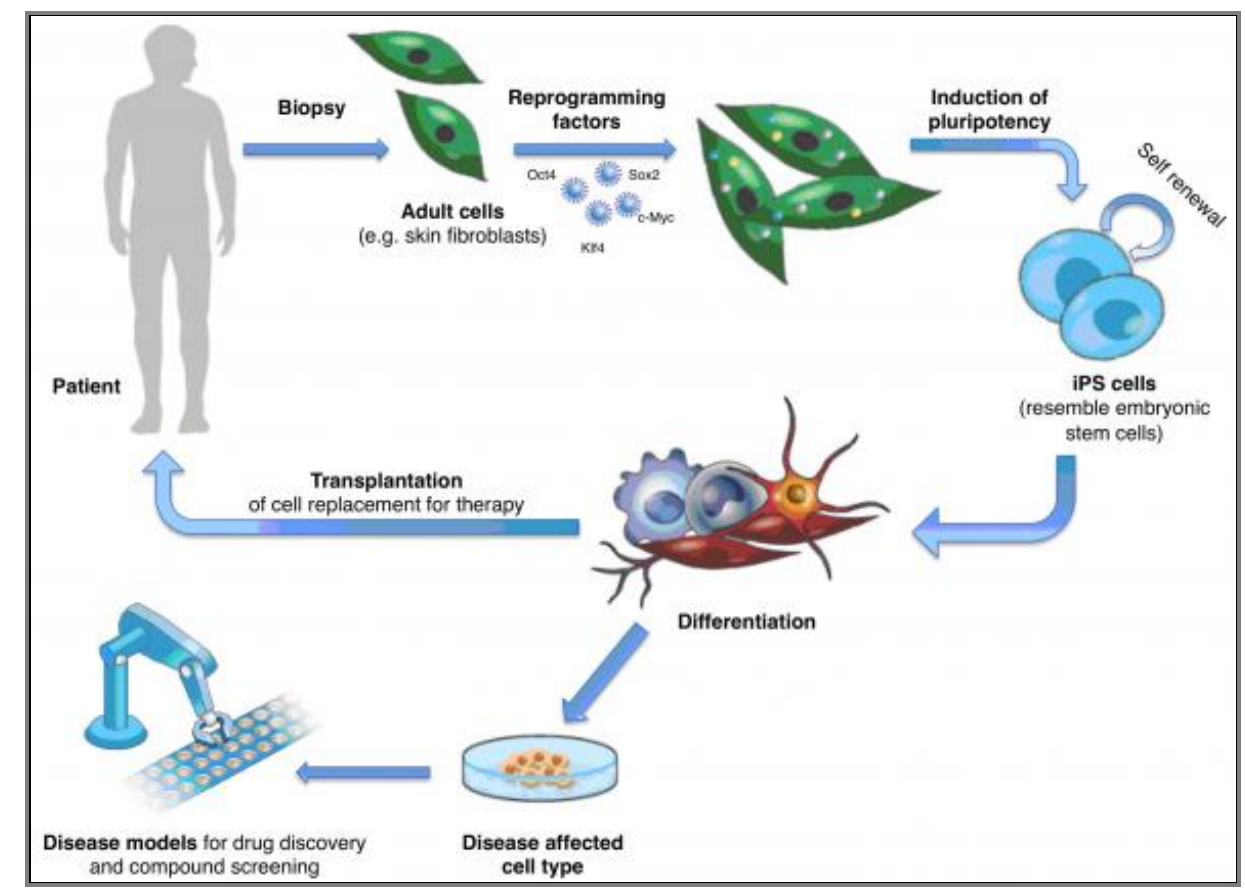

Figure 2.3. The derivation and applications of iPS cells. The iPS cells can be generated from patient biopsies. Later on the patient tailored iPS cells are cell-type-specifically differentiated and used for transplantation in cell replacement therapy. Disease-affected cells differentiated from iPS cells can be used for disease modeling and drug discovery. (Figure adapted from: http://www.eurostemcell.org).

Importantly, the iPS technology allows researchers to obtain and to study pluripotent cells without the controversial use of embryos and also to transplant patienttailored cells to avoid any immunogenic responses (Nishikawa et al., 2008). Due to their capacity to differentiate into all kinds of cell types in the body, the use of iPSCs in regenerative medicine is very alluring. Although promising, several studies revealed the existence of minor differences between mouse iPSCs and ESCs at both genetic and epigenetic levels, demanding a need to optimize current protocols (Gore et al., 2011; 
Hussein et al., 2011; Zhao et al., 2011). Of particular interest is the imprinting defect observed at the Dlk1-Dio3 imprinting cluster in majority of iPS cell lines (Stadtfeld et al., 2010). The iPSCs with imprinting defects are developmentally compromised and show reduced chimerism with loss of germ-line transmission capacity (Stadtfeld et al., 2010; Liu et al., 2010). Taken together, these results suggest that there are still many unknown flaws with iPSCs and a complete characterization should be performed before consideration of any clinical applications.

\subsection{Objectives}

To better understand the role of germ cell marker genes in establishment and maintenance of pluripotency, the current study was designed and performed with following objectives:

1. Expression analysis of selected stage-specific germ cell marker genes in various pluripotent cell types at the genetic and epigenetic level.

2. Analysis of selected stage-specific germ cell marker genes expression during the process of iPS cells generation.

3. Role of germ stem cell marker gene, Dppa3, in establishment of pluripotency during somatic cell reprogramming.

4. Elucidating the function of mouse Dazl and its novel splice variant in pluripotent stem cells. 


\section{Results}

The present thesis systematically analyzed the expression of selected stage-specific germ cell marker genes in pluripotent cells and studied their functions in establishment and induction of pluripotency. To gain more insights into the molecular mechanism, two representative marker genes, Dppa3 (germ stem cell marker) and Dazl (pre-meiotic maker) were selected and their roles in pluripotency were elucidated separately. The results of this thesis are summarized in the three following manuscripts, where the first one has been published, the second and the third have been submitted to peer-reviewed journals.

3.1. Stage-specific germ-cell marker genes are expressed in all pluripotent cell types and emerge early during induced pluripotency.

3.2. Dppa3 binds to the IG-DMR of the Dlk1-Dio3 imprinting cluster and prevents its imprinting loss during iPS cell generation.

3.3. Mouse Dazl and its novel splice variant function in translational repression of target mRNAs in embryonic stem cells. 
3.1. Stage-specific germ cell marker genes are expressed in all pluripotent cell types and emerge early during induced pluripotency

In the first part of the thesis, using mouse as a model system, we systematically studied RNA and protein expression of germ stem cell/pre-meiotic (GC/PrM) markers in ESCs, iPSCs and germ line derived pluripotent stem cell lines such as EGCs, ECCs and maGSCs. Furthermore, by taking the advantage of gene silencing technique, we showed that the GC/PrM networks are independent from pluripotency network comprising of Oct3/4, Sox 2 and Nanog in ESCs. Strikingly, the chromatin immunoprecipitation (ChIP) assay revealed an active chromatin structure for GC marker genes and a bivalent chromatin states at PrM marker genes. Additionally, we studied the gene expression pattern during the time course of induced pluripotent stem cells generation from fibroblast cells and identified that GC marker genes are expressed earlier than that of pluripotency marker genes. To sum up, based on our results, we speculate that the expression of GC markers might indicate the germ cell origin of ESCs whereas the PrM marker genes expression might indicate the poised state of pluripotent stem cells for germ line commitment.

Authors: Xingbo Xu, D.V.Krishna Pantakani, Sandra Lührig, Xiaoying Tan, Tatjana Khromov, Jessica Nolte, Ralf Dressel, Ulrich Zechner and Wolfgang Engel

Status: Published in PLoS ONE 6(7): e22413. doi:10.1371/journal.pone.0022413

\section{Impact Factor: 4.351}

\section{Author contributions to the work:}

1. Xingbo Xu: Conception and design of experiments, data collection, data analysis, data interpretation, helped in drafting the manuscript.

2. D.V. Krishna Pantakani: Conception and design of experiments, data analysis, data interpretation, drafted the manuscript.

3. Sandra Lührig: Performed Western blot and RT-PCR assay, data analysis.

4. Xiaoying Tan: Data collection, data analysis.

5. Tatjana Khromov: Performed Chip assay, data analysis.

6. Jessica Nolte: Data collection, data analysis.

5. Ralf Dressel: Performed teratoma formation assay, data analysis.

6. Ulrich Zechner: Performed DNA methylation assay, data analysis, helped in drafting the manuscript.

7. Wolfgang Engel: Conception and design of experiments, financial support. 


\title{
Stage-Specific Germ-Cell Marker Genes Are Expressed in All Mouse Pluripotent Cell Types and Emerge Early during Induced Pluripotency
}

\author{
Xingbo Xu' ${ }^{1}$, D. V. Krishna Pantakani ${ }^{1 *}$, Sandra Lührig' ${ }^{1}$, Xiaoying Tan ${ }^{1}$, Tatjana Khromov' ${ }^{1}$ Jessica Nolte', \\ Ralf Dressel $^{2}$, Ulrich Zechner ${ }^{3}$, Wolfgang Engel ${ }^{1 *}$
}

1 Institute of Human Genetics, University of Goettingen, Goettingen, Germany, 2 Department of Cellular and Molecular Immunology, University of Goettingen, Goettingen, Germany, 3 Institute of Human Genetics, University of Mainz, Mainz, Germany

\begin{abstract}
Embryonic stem cells (ESCs) generated from the in-vitro culture of blastocyst stage embryos are known as equivalent to blastocyst inner cell mass (ICM) in-vivo. Though several reports have shown the expression of germ cell/pre-meiotic (GC/ PrM) markers in ESCs, their functional relevance for the pluripotency and germ line commitment are largely unknown. In the present study, we used mouse as a model system and systematically analyzed the RNA and protein expression of GC/PrM markers in ESCs and found them to be comparable to the expression of cultured pluripotent cells originated from the germ line. Further, siRNA knockdown experiments have demonstrated the parallel maintenance and independence of pluripotent and GC/PrM networks in ESCs. Through chromatin immunoprecipitation experiments, we observed that pluripotent cells exhibit active chromatin states at GC marker genes and a bivalent chromatin structure at PrM marker genes. Moreover, gene expression analysis during the time course of iPS cells generation revealed that the expression of GC markers precedes pluripotency markers. Collectively, through our observations we hypothesize that the chromatin state and the expression of GC/PrM markers might indicate molecular parallels between in-vivo germ cell specification and pluripotent stem cell generation.

Citation: Xu X, Pantakani DVK, Lührig S, Tan X, Khromov T, et al. (2011) Stage-Specific Germ-Cell Marker Genes Are Expressed in All Mouse Pluripotent Cell Types and Emerge Early during Induced Pluripotency. PLoS ONE 6(7): e22413. doi:10.1371/journal.pone.0022413

Editor: Eliana Saul Furquim Werneck Abdelhay, Instituto Nacional de Càncer, Brazil

Received April 28, 2011; Accepted June 21, 2011; Published July 25, 2011

Copyright: $\odot 2011$ Xu et al. This is an open-access article distributed under the terms of the Creative Commons Attribution License, which permits unrestricted use, distribution, and reproduction in any medium, provided the original author and source are credited.

Funding: This work was partly supported by the German Research Foundation (Deutsche Forschungsgemeinschaft) DFG SPP1356 (EN 84/22-1; ZE 442/4-1) and DFG FOR 1041 (EN 84/23-1; ZE 442/5-1). The funders had no role in study design, data collection and analysis, decision to publish, or preparation of the manuscript.

Competing Interests: The authors have declared that no competing interests exist.

*E-mail: krishna.if1@gmail.com (DVKP); wengel@gwdg.de (WE)
\end{abstract}

\section{Introduction}

The capacity of long-term self-renewal and the unique ability to differentiate into all three germ layer cell types (ectoderm, endoderm and mesoderm) define pluripotency. According to the source of cells used for the establishment of pluripotent cells, one can currently distinguish between the following pluripotent cell lines: (1) embryonic stem cells (ESCs) derived from the inner cell mass of mouse blastocysts at embryonic day 3.5 (E3.5) [1,2], (2) embryonic germ cells (EGCs) cultured from primordial germ cells (PGCs) that colonize the genital ridge at E12.5 [3,4], (3) embryonal carcinoma cells (ECCs) isolated from germ-cell tumors of either testis or ovary [5], (4) germ line stem cells isolated from mouse neonatal and adult testis (GSCs and maGSCs, respectively) $[6,7]$ and (5) induced pluripotent stem cells (iPSCs), derived from reprogramming somatic cells by ectopic expression of defined transcription factors [8]. All the above mentioned cultured pluripotent cell lines (EGCs, ECCs, GSCs, and maGSCs) have a germ-cell origin, except ESCs, whose origin is not clearly understood. Although these cell lines have different molecular profiles mostly due to their developmental stage of isolation, they share the expression of germ cell/pre-meiotic (GC/PrM) markers that may indicate a germ-cell origin [9].
During embryonic development, the specification of PGCs is crucial for the development of the germ line, which is finally destined to give rise to the totipotent zygote upon fertilization. Prior to gastrulation, the precursors of primordial germ cells arise in the E6.25 proximal epiblast from 48 cells positive for the transcriptional repressor Blimpl [10,11]. These Blimpl-positive cells continuously proliferate and start to express other PGC markers such as Fragilis and Stella by E7.5. Thereafter, PGCs initiate migration and colonization of the genital ridge and increase their number to approximately 4000 by E12.5 [12,13]. Further development of PGC/germ cells to mature spermatozoa or oocytes depends on the coordinated genetic and epigenetic events [14]. Interestingly, several studies have demonstrated the expression of some of the GC/PrM markers like Blimpl, Stella, Fragilis, Piwil2, Dazl and MVH in ES cells at the RNA level $[15,16,17]$, raising the possibility that ES cells might originate from the germ line [9].

In the present study, using mouse as a model system, we have systematically analyzed the expression of GC/PrM markers in ES cells compared to germ line origin cultured pluripotent cells like EGCs, ECCs, GSCs and maGSCs and found comparable expression at the RNA and protein level. Moreover, we show the expression of Stella, Dazl and MVH in preimplantation 
embryos and, the independence of pluripotency-specific networks from germ cell-specific networks in ES cells. Interestingly, chromatin immunoprecipitation (ChIP) analysis revealed that ES cells exhibit active chromatin states at GC marker genes and a bivalent chromatin structure at PrM marker genes. Further, gene expression analysis during iPSC generation revealed that the expression of GC markers precedes pluripotency markers. Collectively, our data indicates the possible link between in-vivo germ cells specification and in-vitro pluripotent stem cells generation.

\section{Materials and Methods}

\section{Cell culture}

Derivation and maintenance of male mouse ESC and maGSC lines from different genetic backgrounds (129Sv and C57BL/6) were described previously [18]. The female ESC line ES Rosa26 was generated from Rosa26-LacZ transgenic mouse line as described for MPI-VI, a previously generated female ESC line [19]. iPS cells (O18) were a kind gift from Prof. Rudolf Jaenisch [20]. All above cell lines including EGC line (EG 1) and parthenogenetic cells were maintained in standard ESC culture conditions. ECC cell line (F9) protein extract was provided by Mr. Peter Christalla, Goettingen. For knockdown experiments, ES cells were seeded in KO-DMEM supplemented with KO-serum replacement (Invitrogen) at a density of $2 \times 10^{5}$ cells $/ \mathrm{ml}$ on feeder layer. After $5 \mathrm{~h}$ of plating, the cells were transfected with either Dazl siRNA (NM_010021.4_stealth_199,_726,_1056, Invitrogen) or MVH siRNA (NM_001 145885.1_stealth_83,_922, _1599, Invitrogen) or Oct3/4 siRNA (NM_013633_Stealth_356, _463, _727, Invitrogen) or scrambled siRNA (Control siRNA, Invitrogen) using Lipofectamine2000 (Invitrogen) according to manufacturer's instructions. After $3 \mathrm{~h}$ of transfection, the medium was changed to standard ES culture medium and allowed to grow for $24 \mathrm{~h}$. The next day, transfection was repeated and cells were harvested after additional $24 \mathrm{~h}$ of culture.

All animal experimentations were reviewed and approved by the Institutional Animal Care and Use committee of the University of Goettingen (Approval ID: 33.9.42502-097/06).

\section{Generation of iPS cells}

We used Yamanaka factors (retroviral expression vectors for Oct3/4, Sox2, Klf4, c-Myc) to generate iPS cells [8]. For reprogramming studies, embryonic fibroblasts isolated from transgenic Nanog-EGFP mice [21] were transduced with retroviral particles as previously described [8]. To establish iPS cell lines, colonies which appeared after 10 days of virus infection were picked manually and cultured in 24-well plates containing feeder layer with standard ES medium and were monitored for the NanogEGFP activation using invert microscope (Olympus). For time course experiments, all cells from culture plates were collected on days 0 (Control), 5-10, 12, 14, 16, 18, 20 and 22 after viral infection for total RNA isolation.

\section{RNA extraction, RT-PCR and qPCR}

Total RNA was extracted from cells using Trizol Reagent (Invitrogen) and from $\sim 50$ blastocysts using Picopure Kit (Analytical Technology) following the manufacturers' protocols. Total RNA from blastocysts or $5 \mu \mathrm{g}$ of total RNA from cells was digested with DNaseI (Sigma) and used for cDNA synthesis using the SuperScriptII system (Invitrogen). For qPCR analysis, diluted cDNA $(1 / 20)$ was used as a template in a Platinum SYBR Green qPCR SuperMix-UDG with ROX (Invitrogen) and run in ABI 7900HT Real-Time PCR System (Applied Biosystems). Primers used in RT-PCR and ${ }_{\mathrm{q}} \mathrm{PCR}$ are listed in supplementary tables (Tables S1, S2, S3).

\section{Protein extraction and Western blotting}

Proteins were extracted from cells and tissues using lysis buffer (10 mM Tris- $\mathrm{HCl}$ (pH 8.0), $1 \mathrm{mM}$ EDTA, 2.5\% SDS, $100 \mathrm{mM}$ PMSF) containing protease inhibitor cocktail (Roche). Protein samples were resolved on 4-12\% SDS-PAGE and transferred onto nitrocellulose membrane (Amersham Biosciences). Membranes were processed using standard Western blot protocols, and signals detected using a chemiluminescent kit (Santa Cruz Biotechnology). Antibody sources are listed in supplementary tables (Table S5).

\section{Early-stage-embryo collection, immunocytochemistry,} and alkaline phosphatase staining

Early stage embryos collected from the oviduct or uterus of pregnant CD-1 mice were used for whole-mount immunostaining as described previously [22]. Immunostained embryos were mounted on cavity microscope slides using Vectashield DAPI mounting medium (Vector Laboratories) and images were acquired using an Olympus confocal microscope. Immunostaining on iPS cells using SSEAl antibody was performed as previously described [23]. Alkaline phosphatase (AP) staining was performed according to manufacturer's protocol (Sigma).

\section{Chromatin immunoprecipitation}

The chromatin immunoprecipitation (ChIP) was performed essentially as previously described [23]. Briefly, cultured wild-type ESCs were cross-linked with $1 \%$ formaldehyde and briefly incubated in lysis buffer followed by sonication (Branson Sonifier 250). Soluble chromatin was pre-cleared with protein-A sepharose beads and incubated with and without (negative-control) antisera (3-5 $\mu \mathrm{g})$ directed against H3K4me3, H3K9ac, H3K9me3, and H3K 27 me3. Enriched DNA was analyzed by qPCR using SYBR green (Invitrogen)-based PCR amplification with primers listed in supplementary tables (Table S4). qPCR data from two or more biological replicates were calculated and expressed as percentage of input DNA precipitation. Antibodies used in ChIP are listed in supplementary tables (Table S5).

\section{Teratoma formation assay}

Teratoma formation assay was performed as previously described $[24,25]$. Briefly, iPS cells $\left(1 \times 10^{6}\right.$ cells $)$ were injected subcutaneously into 8 to 10 weeks old female severe combined immunodeficient $\mathrm{RAG}^{-/-} \mathrm{c} \gamma \mathrm{c}^{-/-}$mice lacking $\mathrm{T}, \mathrm{B}$, and natural killer (NK) cells. Tumor growth was monitored weekly by palpation and size was recorded using linear calipers. Animals were sacrificed when a tumor diameter of $1 \mathrm{~cm}$ was reached. Autopsies were performed and tumor tissue was placed in phosphate-buffered $4 \%$ formalin for $16 \mathrm{~h}$ and then embedded in paraffin. For histological analysis, the specimens were stained with haematoxylin and eosin (HE).

\section{Statistical Analysis}

All qPCR data for RNA expression analysis (two or more biological replicates) were calculated using standard curve method. For statistical significance calculations, 2way ANOVA (GraphPad Prism 4.0) test was used.

\section{Results}

\section{Pluripotent stem cells express GC/PrM genes}

To investigate whether GC/PrM gene expression is characteristic of all known mouse pluripotent cells, we systematically analyzed several pluripotent cells. Firstly, we examined GC/PrM gene expression in male maGSCs and ESCs from different genetic 
backgrounds and in iPSCs, EGCs, and F9 cells by Western blotting (Fig. 1A). GC markers Stella and Fragilis were readily detected in all cell types (Fig. 1A), including parthenogenetic cells (Fig. 1B). Further, PrM markers Piwil2, Dazl, and MVH were found to be expressed in all pluripotent cells, except ECCs (Fig. 1A). Protein levels of GC/PrM markers were reduced or absent in ESCs and maGSCs upon spontaneous differentiation with retinoic acid for 20 days (Fig. 1B). Overmore, we analyzed multipotent mesenchymal stem cells (MSCs) and could not detect any expression of GC/PrM markers (Fig. 1B). We also performed RT-PCR analysis for other PrM, meiotic, and post-meiotic markers (Fig. 1C). Expression of PrM markers Stra8, Rnf17, Rnh2, and Piwil2 was detected in all cells (Fig. 1C). Surprisingly, meiotic markers $S y c p 3, P g k 2$, and $C r e b 3 / 4$ were also detected in all pluripotent cell lines (Fig. 1C). However, expression of several other developmental markers such as post-meiotically expressed Tp2, Theg, Gpx4, Pmml, and mature spermatozoan marker Cylcl was undetectable as expected (Fig. 1C).

To determine whether the expression of GC/PrM markers is specific to male pluripotent cells, we studied two female ES cell lines, namely, MPI VI and ES Rosa26. Pluripotency of these cell lines was confirmed by detecting the expression of the key pluripotency markers Oct3/4 and Sox2 (data not shown). Both female pluripotent cell lines were found to express all analyzed $\mathrm{GC} / \mathrm{PrM}$ markers with levels similar to those of male pluripotent cells (Fig. 1D).

GC/PrM genes are also expressed in early embryogenesis

Next, we studied the expression of GC marker (Stella) and PrM markers (Dazl and MVH) in early stages of mouse embryogenesis (2-, 4-, 8-cell stages) by immunocytochemistry (ICC) (Fig. 2A). Interestingly, we found Stella, Dazl and MVH to be expressed throughout all stages of embryogenesis (Fig. 2A). To determine the expression levels of $\mathrm{GC} / \mathrm{PrM}$ markers at the blastocyst stage, we performed $\mathrm{qPCR}_{\mathrm{PC}}$ on blastocyst stage embryos (Fig. 2B). In agreement with our ICC results, all analyzed GC/PrM markers (Fragilis, Dazl, MVH, and Strag) were detected at the blastocyst stage with transcript levels, that are, however, markedly lower than those of pluripotency markers such as Oct3/4, Nanog, Lin28 (Fig. 2B).

\section{Independency of pluripotent and GC/PrM networks in} ESCS

The widespread expression of GC/PrM markers in pluripotent cells led us to study their influence on other GC/PrM and key pluripotency markers (Fig. 3). Firstly, we down-regulated Dazl in ES cells using siRNA and found an $\sim 80-90 \%$ decrease at both the RNA and protein level (Fig. 3A, B). In contrast, control siRNA treated cells did not exhibit altered Dazl expression levels (Fig. 3A, B). Then, we performed a qPCR-based analysis of expression levels of key pluripotency markers and detected no significant differences among control siRNA treated and Dazl siRNA treated cells (Fig. 3C). Similarly, the expression of PrM markers $M V H$ and Stra8 did not change significantly, whereas GC markers (Stella and Fragilis) showed significant up-regulation in Dazl down-regulated cells (Fig. 3D). Similarly, $\sim 70 \%$ down-regulation of $\mathrm{MVH}$ expression (Fig. 3E, F) did not influence expression of key pluripotency markers (Fig. 3G). Consistent with Dazl downregulation, MVH depletion had no effect on Dazl and Stra8, but Stella and Fragilis were significantly up-regulated (Fig. 3H). Conversely, we down-regulated $O c t 3 / 4$ and studied the expression of GC/PrM and pluripotency markers. The down-regulation of $O c t 3 / 4$ resulted in significant down-regulation of Klft expression, whereas the expression of other pluripotent markers such as Nanog,
Z $p 206$, and Lin28 did not alter (Supplementary Fig. S1). Furthermore, the down-regulation of $O \epsilon \mid 3 / 4$ had no statistically significant effect on the expression of $\mathrm{GC} / \mathrm{PrM}$ markers (Supplementary Fig. S1).

Active chromatin at GC marker gene promoter regions and bivalent chromatin at PrM marker gene promoters in ESCs and iPSCs

We hypothesized that the chromatin state at the promoter regions of $\mathrm{GC} / \mathrm{PrM}$ markers might elucidate their role in the establishment/maintenance of pluripotency or lineage specification in ESCs. We analyzed the ChIP sequencing data of mouse ES cells, which is freely available [26] and found that the promoter regions of GC markers Blimpl, Stella and Fragilis were enriched for H3K 4me3 indicating the transcriptionally active chromatin state, as seen for Oct3/4 (Supplementary Fig. S2A). In contrast the promoter regions of $D a z l$ and $M V H$ were decorated with both H3K4me3 and H3K27me3, highlighting the bivalent chromatin state, which is a hall mark of lineage specification genes, such as Hoxall and Pax5 (Supplementary Fig. S2B). To further validate these observations, gene specific histone modification profiles (active: H3K4me3, H3K9ac; and repressive: H3K9me3, $\mathrm{H} 3 \mathrm{~K} 27 \mathrm{me} 3$ ) were analyzed by ChIP at the promoter regions of GC markers Fragilis and Blimpl, and PrM markers Dazl and MVH, and compared to the promoter regions of $O c t 3 / 4$ (transcriptionally active chromatin) and Hoxall and Pax 5 (bivalent chromatin) in ES cells (Fig. 4A). qPCR quantification of ChIP DNA showed that the promoter regions of GC markers Fragilis and Blimpl were enriched for the activating modifications $\mathrm{H} 3 \mathrm{~K} 4 \mathrm{me} 3$ and $\mathrm{H} 3 \mathrm{~K} 9 \mathrm{ac}$, but depleted for the repressive modifications H3K9me3 and H3K27me3, indicating a transcriptionally active chromatin similar to key pluripotency Oct3/4 gene promoter (Fig. $4 \mathrm{~A}$ ). In contrast, the promoters of PrM genes Dazl and $M V H$ were enriched for both active (H3K4me3 and H3K9ac) and repressive (H3K27me3) modifications, representing the bivalent chromatin domain similar to lineage specific genes (Hoxall and Pax5) (Fig. 4A). Moreover, we also performed gene specific histone modification profiling in established iPS cells [20] and found similar results like ES cells (Fig. 4B).

\section{GC markers emerge during early reprogramming of MEFs into iPSCs}

To further understand the role of GC/PrM markers during the establishment and maintenance of pluripotency, we used ectopic expression of the four Yamanaka factors (Oct4, c-Myc, Klf4, and Sox2) for reprogramming of somatic cells to induced pluripotency. Firstly, we reprogrammed MEFs isolated from Nanog-EGFP mice and established four iPS lines (xul, 2, 5, and 6), which are morphologically similar to ES cells, activate the Nanog promoterdriven EGFP expression, positive for AP staining, SSEAl immunostaining and express endogenous Oct $3 / 4$ and Sox2 (Fig. 5A-D). The iPSC lines were further characterized by histone modification, and DNA methylation profiling of key pluripotent marker genes (data not shown). Finally, all the iPSC lines (xu1, 2, 5 and 6) were injected subcutaneously into immunodeficient mice. Two recipients were used per cell line. In all mice, tumors were observed and the mice had to be sacrificed between day 18 and day 33 after injection. The tumors were identified as teratomas by histological examination as exemplified for iPS cell lines xu2 and 6 (Supplementary Fig. S3). Then, we setup a time course experiment and analyzed the expression levels of key pluripotency genes and $\mathrm{GC} / \mathrm{PrM}$ genes during the course of reprogramming as outlined in figure $5 \mathrm{E}$. Expression analysis using real time $\mathrm{qPCR}$ revealed 

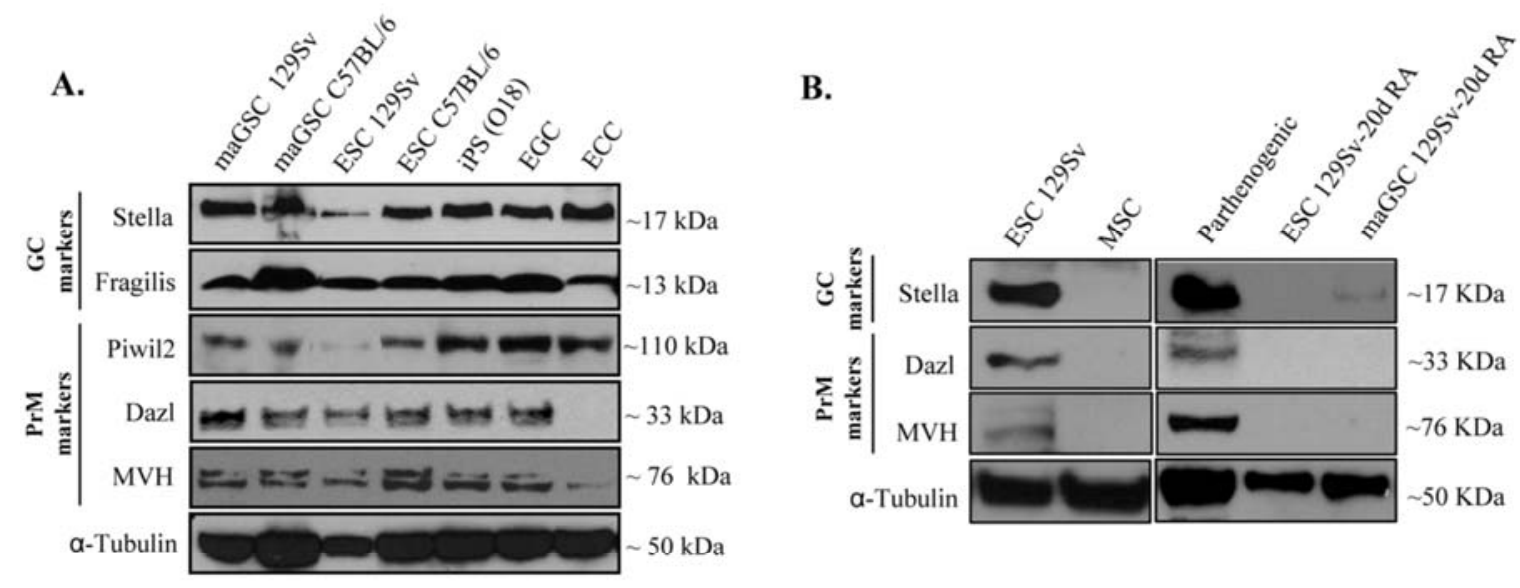

C.
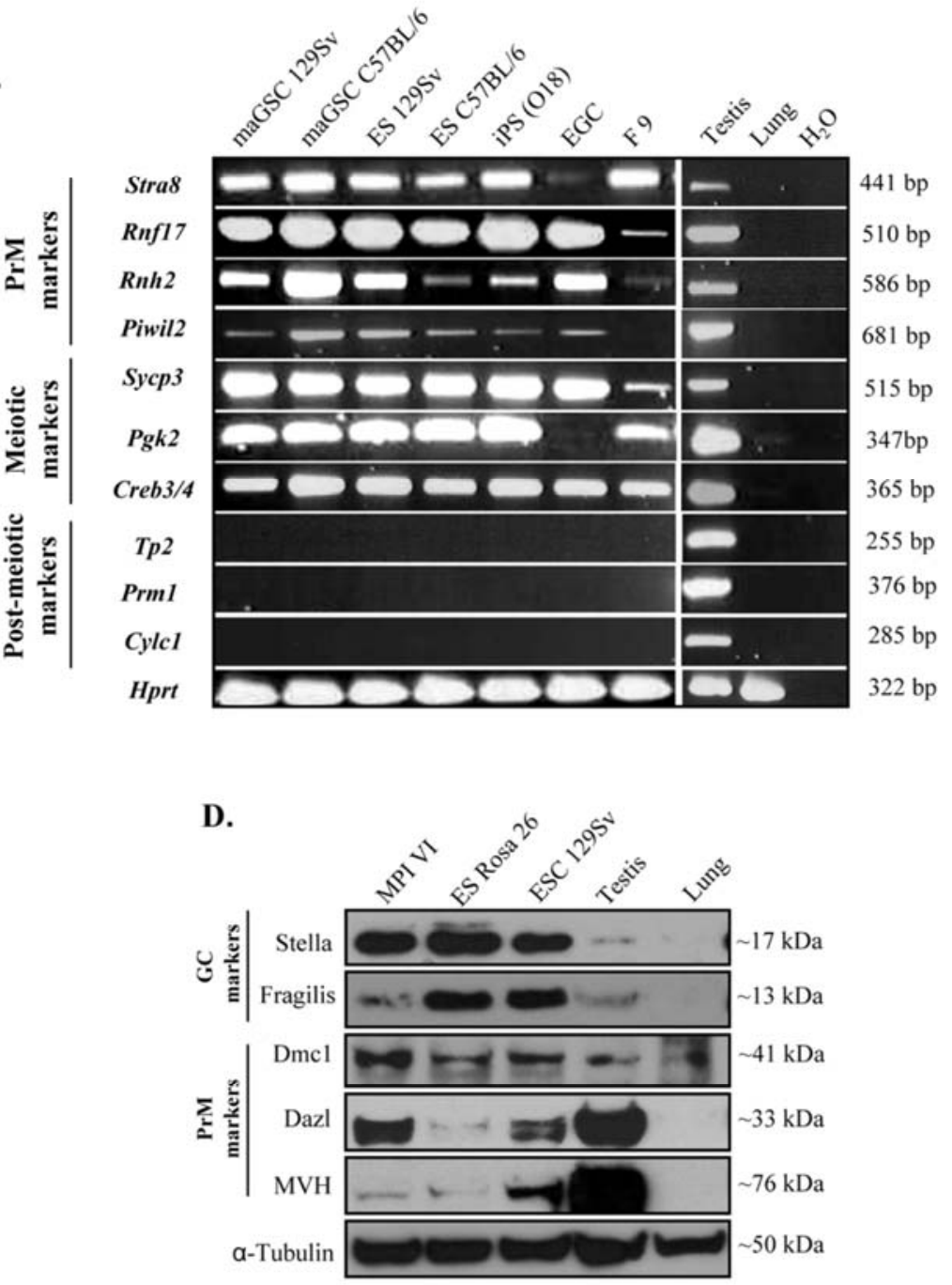

(2). PLOS ONE | www.plosone.org 
Results

Germ-Cell Markers in Pluripotent Cells

Figure 1. Expression analysis of GC/PrM genes in pluripotent cell lines. (A) Expression of GC markers (Stella, Fragilis) and PrM markers (Piwil2, Dazl, MVH) in maGSCs and ESCs (of 129Sv and C57BL/6 genetic backgrounds), iPSCs, EGCs and ECCs. (B) Western blot of GC marker (Stella) and PrM markers (Dazl and MVH) in mouse mesenchymal stromal cells (MSC), parthenogenetic cells, ES and maGSC treated with retinoic acid for 20 days. Protein extract from ESCs was used as a positive control. (C) RT-PCR analysis showing the expression of PrM (Stra8, Rnf17, Rnh2 and Piwil2), meiotic (Sycp3, Pgk2 and Creb3/4) and post-meiotic (Tp2, Prm1 and Cylc1) markers in maGSC and ESC (from different genetic backgrounds), as well as iPSC, EGC and ECC cell lines. CDNA from wild type mouse testis and lung were used as positive and negative controls, respectively. (D) Western blot analysis showing the expression of GC markers (Stella, Fragilis), PrM markers (Dmc1, Dazl, MVH) in two different female ESC lines (MPI VI, ES Rosa26). $\alpha$-tubulin was used as loading control in A, B and D.

doi:10.1371/journal.pone.0022413.g001

significant expression levels of key germ cell markers (Blimp1 and Fragilis) at day 6 and a gradual increase to the levels seen in ES cells by day 22 (Fig. 5F, G). Transcripts of Stella, another germ cell marker, were significantly detectable at day 10 of reprogramming and reached levels similar to those in ES cells by day 22. (Fig. 5F, G). In contrast, significant endogenous expression levels of the key pluripotency markers $O c t 3 / 4$ and Sox 2 occurred only on day 12 of reprogramming and showed expression levels typical for ES cells by

A.

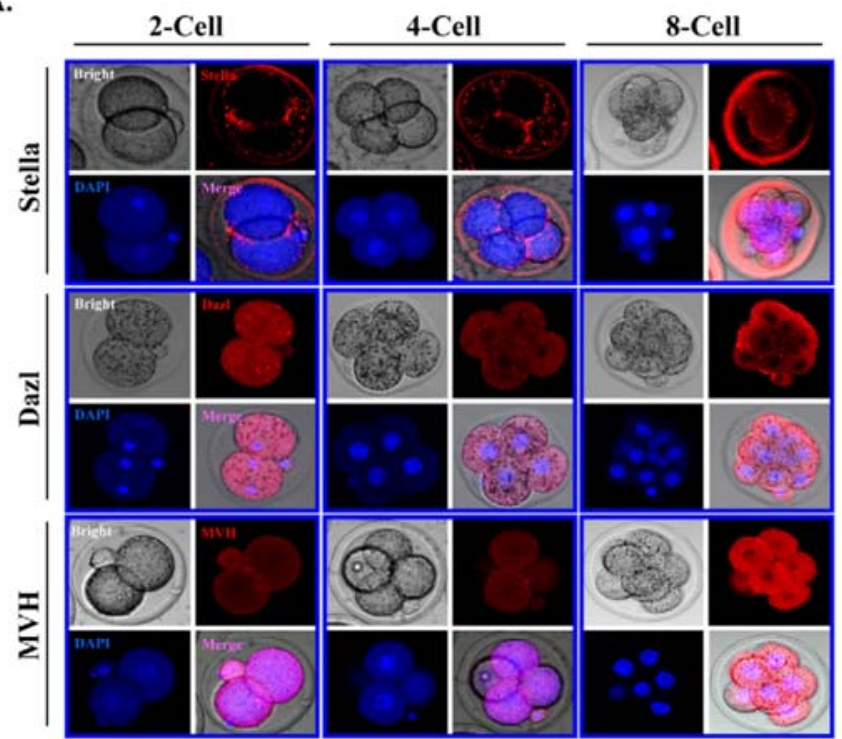

B.

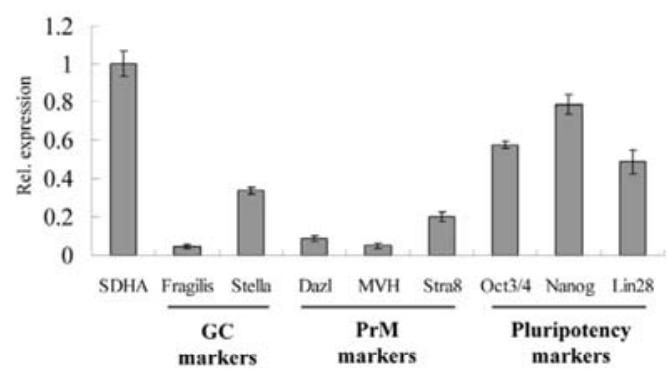

Figure 2. Expression analysis of GC/PrM genes in early embryogenesis. (A) Immunocytochemistry showing the expression of GC marker (Stella) and PrM markers (Dazl and MVH) in pre-implantation embryos (2-cell, 4-cell, and 8-cell stage). DAPI was used as a counter stain to visualize the nucleus. (B) Quantitative real time PCR analysis was used to evaluate the expression levels of GC (Fragilis), PrM (Dazl, MVH, Stra8) and pluripotency markes (Oct3/4, Nanog, Lin28) in blastocysts. Expression levels were normalized to Sdha (a house keeping gene). The qPCR data of three biological replicates (including three technical replicates each) were calculated and represented as a mean \pm SD. doi:10.1371/journal.pone.0022413.g002 
A.

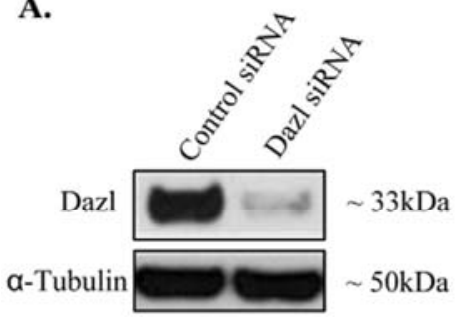

C.

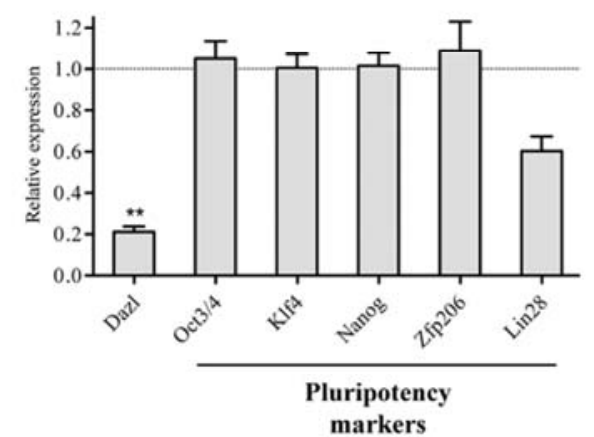

E.

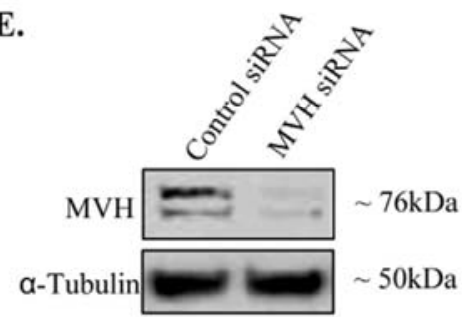

G.

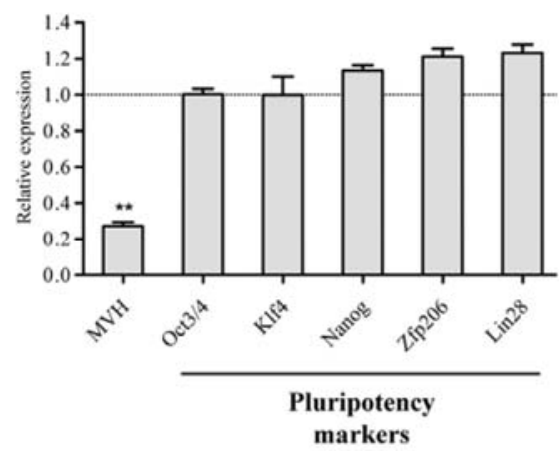

(2). PLOS ONE | www.plosone.org
B.

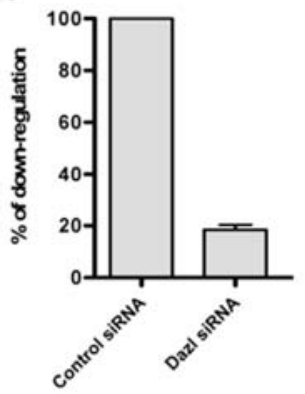

D.

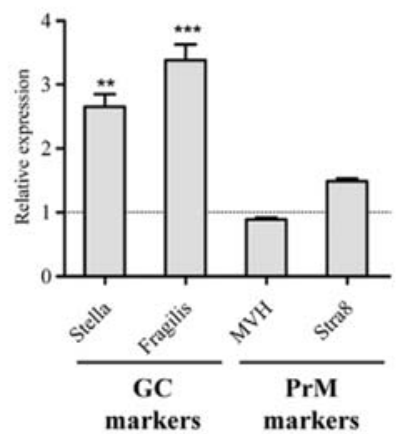

F.

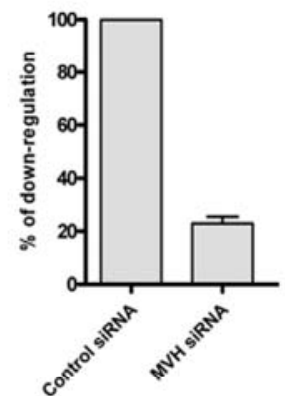

H.

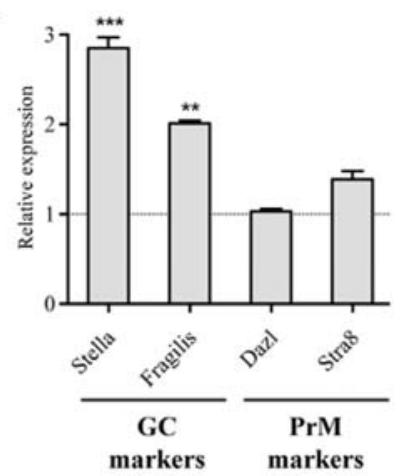


Figure 3. Effect of the downregulation of PrM genes in ESCs. (A and B) Efficiency of Dazl down-regulation as shown by Western blot analysis and subsequent densitometric qunatification. (C) Real time qPCR analysis showing Dazl downregulation at the RNA level and the expression profile of the core pluripotency network (Oct3/4, Klf4, Nanog, Zfp206 and Lin28). (D) Expression of GC (Stella and Fragilis) and PrM (MVH and Stra8) markers in Dazl down-regulated ESCs. (E and F) Western blot showing the efficiency of MVH downregulation and the densitometric quantification, respectively. (G) Real time QPCR analysis showing MVH downregulation at the RNA level and the expression profile of the core pluripotency network (Oct3/4, KIf4, Nanog, Zfp206 and Lin28). (H) Expression of GC (Stella and Fragilis) and PrM (Dazl and Stra8) markers in MVH down-regulated ESCs. The dotted lines in C, D, G, H indicate the normalized expression levels of analyzed genes in control siRNA treated cells. The qPCR data of two biological replicates (including three technical replicates each) were calculated and represented as a mean \pm SD. Expression levels, which are statistically significant, are indicated with asterisks $(* * \mathrm{p}<0.01 ; * * * \mathrm{p}<0.001)$.

doi:10.1371/journal.pone.0022413.g003

day 22 (Fig. 5G). Further pluripotency markers like Z Jp206 and Nanog appeared only on day 18 and 20, respectively and increased to levels observed in ES cells only in fully reprogrammed and established iPS cells (Fig. 5G). Surprisingly, we could not detect significant expression of pre-meiotic markers such as Stra8, Dazl and MVH before day 22 of reprogramming. The expression of Stra 8 appeared not until day 22 and the other two markers were only present in established iPS cells (Fig. 5G).

A.

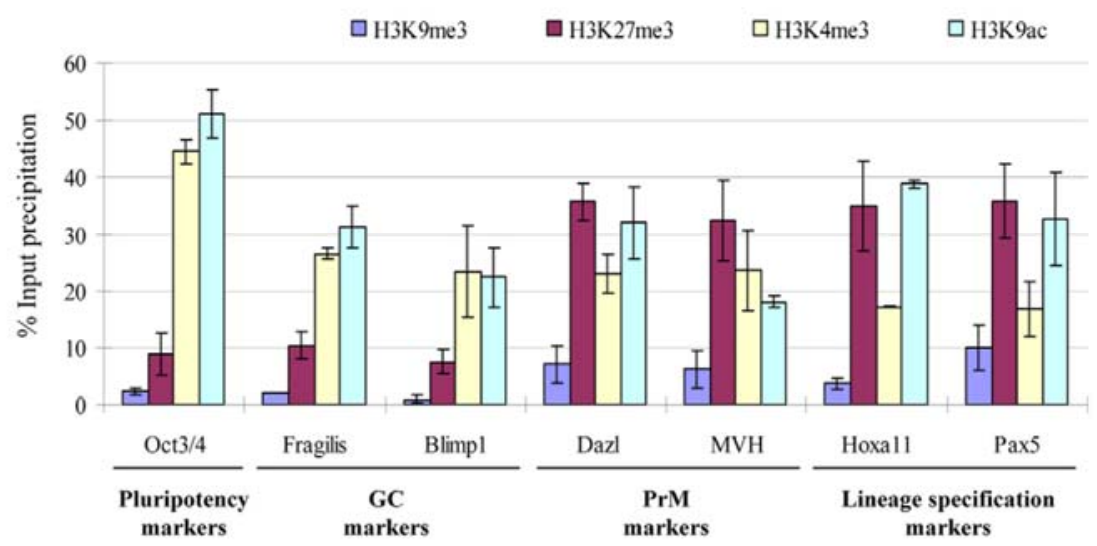

B.

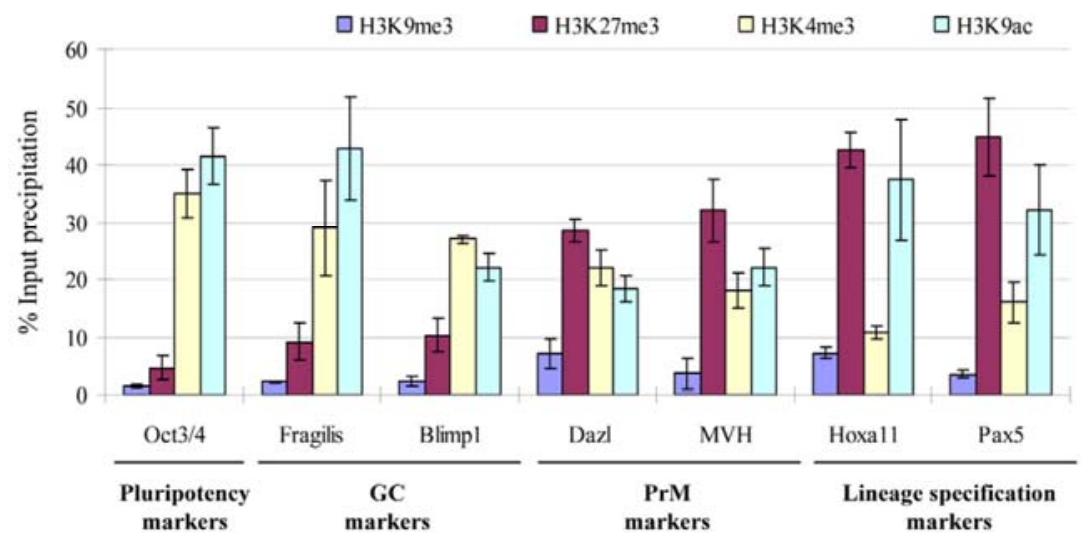

Figure 4. Epigenetic signature of GC/PrM genes in ESCs and iPSCs. ChIP and subsequent real time qPCR for various histone modifications (active: $\mathrm{H} 3 \mathrm{~K} 4 \mathrm{me} 3$ and $\mathrm{H} 3 \mathrm{~K} 9 \mathrm{ac}$; repressive: $\mathrm{H} 3 \mathrm{~K} 9 \mathrm{me} 3$ and $\mathrm{H} 3 \mathrm{~K} 27 \mathrm{me} 3$ ) at the promoter regions of GC (Fragilis and Blimp1) and PrM markers (Dazl and MVH) in ESCs (A) and iPSCs (B). The promoter regions of GC markers are similar to the Oct3/4 promoter and are enriched for active histone modifications, while the promoters of PrM markers are similar to the promoters of the lineage specific genes Hoxa11, Pax5 and were enriched for both active and repressive marks indicating their bivalent chromatin structure. The qPCR data of two biological replicates (including three technical replicates each) were calculated and represented as a mean \pm SD. doi:10.1371/journal.pone.0022413.g004 
A.

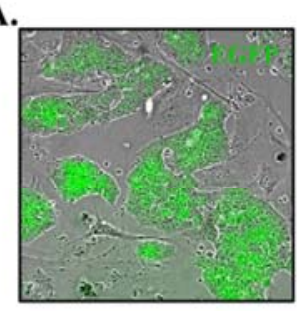

B.

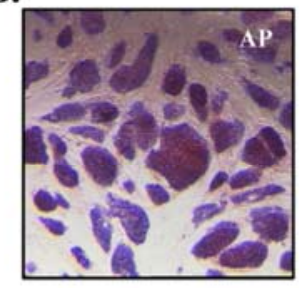

C.

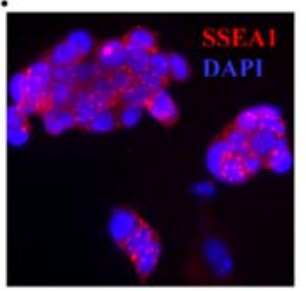

D.

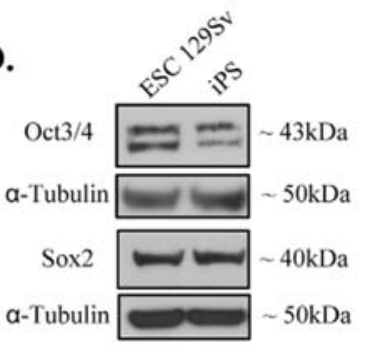

E.

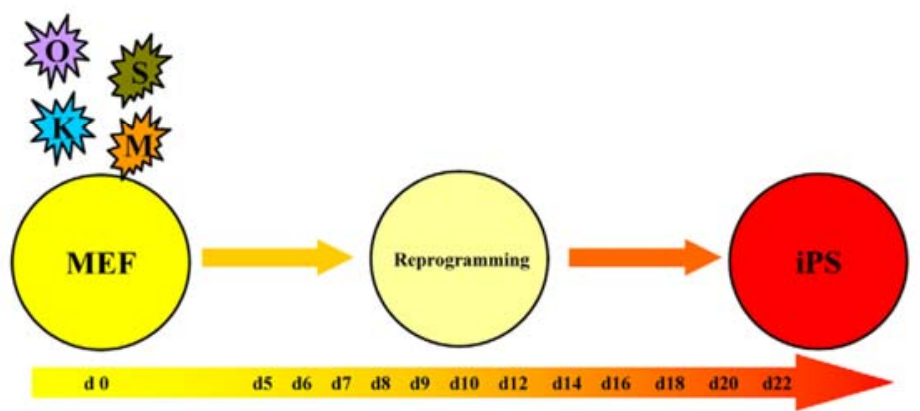

F.

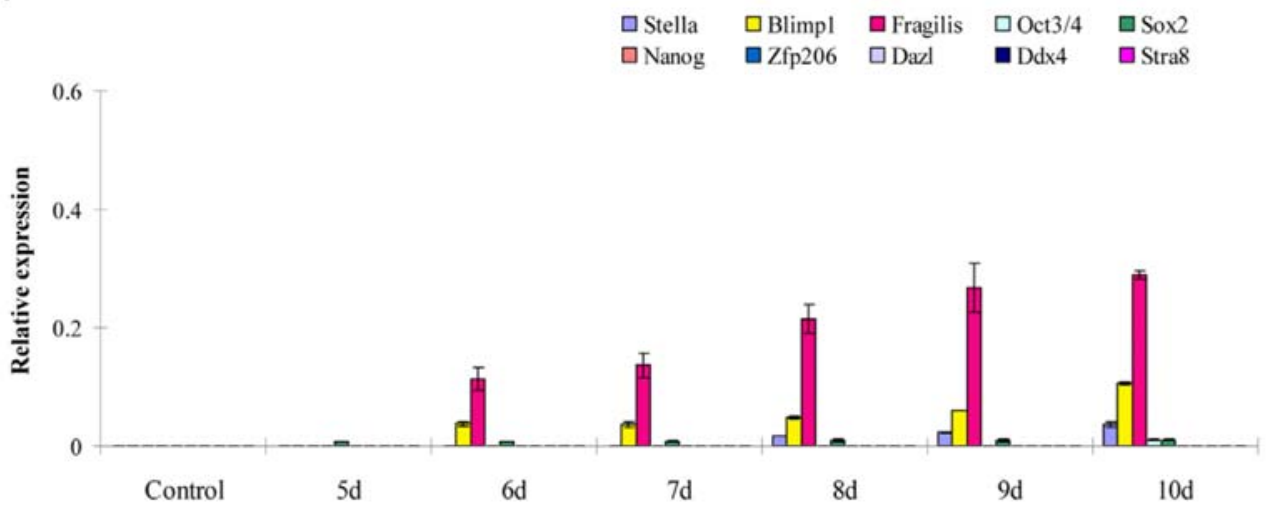

G.

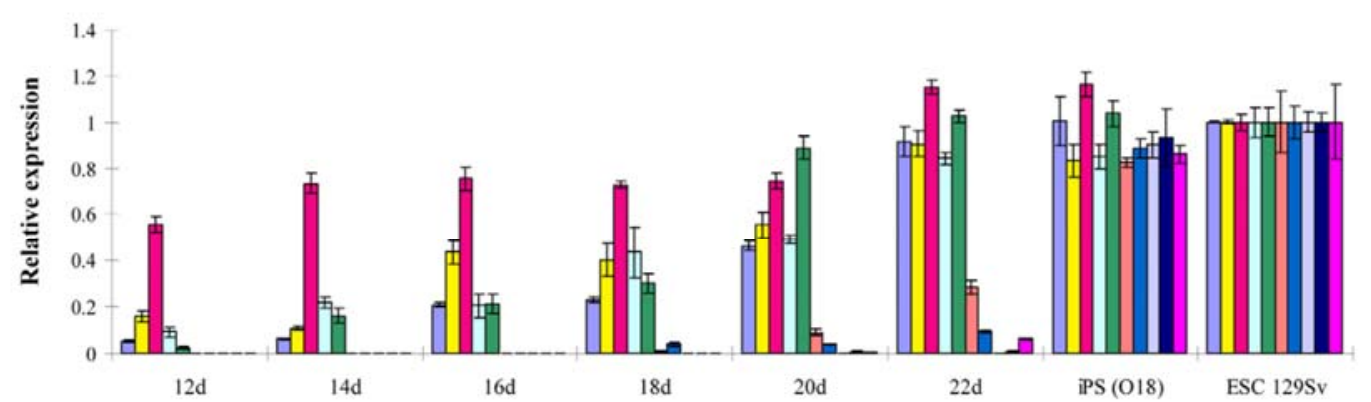

.8. PLOS ONE I www.plosone.org 
Figure 5. Expression pattern of GC/PrM and pluripotency genes during the course of iPS generation. Fully reprogrammed and established iPS cells express Nanog promoter-driven EGFP (A), positive for AP staining (B), and express SSEA1 (C). (D) Expression of key pluripotency markers Oct $3 / 4$ and Sox 2 in iPS cells. Protein extract from ESCs was used as a positive control. (E) Schematic diagram depicting the generation of iPS cells using the four Yamanaka factors and the time course of sample collection (starting from the day of retrovirus transduction, day 0 (d0) till day 22 (d22)) for gene expression analysis. (F) Real time qPCR analysis of pluripotency (Oct3/4, Sox2, Nanog and Zfp206), germ cell (Stella, Blimp1 and Fragilis) and pre-meiotic genes (Dazl, MVH and Stra8) during the time course of iPS cell generation from day5 (5d) to day 10 (10d) after virus infection. NanogEGFP MEFs were used as a control. (G) Real time QPCR analysis for the above mentioned genes during the time course of iPS cell generation from day $12(12 \mathrm{~d})$ to day $22(22 \mathrm{~d})$ after virus infection. The qPCR data of two or more biological replicates (including three technical replicates each) were calculated and represented as a mean \pm SD.

doi:10.1371/journal.pone.0022413.g005

\section{Discussion}

Though traditionally pluripotent ES cells are regarded as in-vitro counterpart of the inner cell mass (ICM), their origin is not yet clearly defined. Recently, it has been hypothesized that ESCs may have a germ-cell origin based on common molecular properties with other pluripotent cells of germ-cell origin [9]. Previously, ES cells were shown to express several GC/PrM markers [15,16,17]. In agreement with these results, that demonstrated the expression of several GC/PrM markers at the transcript level, our Western blot analysis detected the expression of GC/PrM markers in all analyzed pluripotent cell types available including iPS cells and female ES cells of mouse origin. Consistent with an earlier report [27], expression of GC/PrM genes was not detectable in bone marrow-derived multipotent stem cells, thus indicating the unique expression of GC/PrM genes only in pluripotent cells. Our immunocytochemistry expression analysis of GC/PrM markers in preimplantation embryos revealed the expression of Stella, Dazl and MVH in all analyzed preimplantation embryo stages (2-, 4-, 8cell stage). Further, down-regulation of PrM genes in ESCs did not influence the expression levels of pluripotency network genes, but rather increase expression of GC genes. Conversely, downregulation of pluripotency marker Oct $3 / 4$ showed no significant effect on GC/PrM marker genes, thus highlighting the maintenance of parallel but independent networks.

The genome-wide expression profiling of ES cells revealed the expression of a large number of genes at low levels due to the open chromatin state of ES cells leading to leaky expression $[28,29,30,31]$. To elucidate leaky versus essential expression of GC/PrM markers in ES cells, we analyzed the global ChIP-Seq data of ES cells and found an active chromatin state at PGC/germ cell markers and a bivalent chromatin structure at pre-meiotic markers [26]. In support of global ChIP-seq data, our genespecific chromatin state of GC/PrM markers in ES cells confirmed the active chromatin state with enrichment for the activating histone modifications $\mathrm{H} 3 \mathrm{~K} 4 \mathrm{me} 3$ and $\mathrm{H} 3 \mathrm{~K} 9 \mathrm{ac}$ at the promoter regions of PGC markers Blimpl and Fragilis, which demonstrates the fundamental expression of these genes. In contrast, the promoter regions of $\mathrm{Dazl}$ and $\mathrm{MVH}$ were marked with bivalent chromatin state, i.e. enrichment for the two activating (H3K4me3
A.

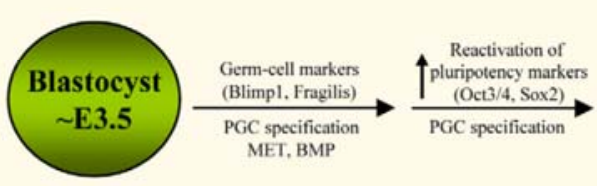

B.

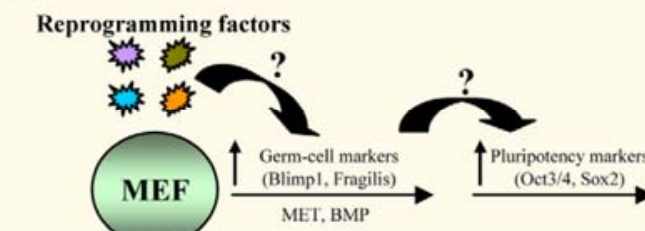

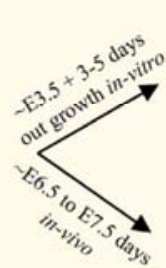
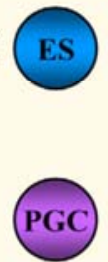

C.

Establishment of pluripotency
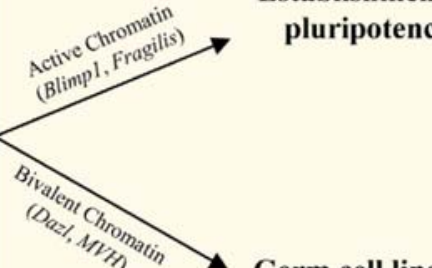

Figure 6. Hypothetical model for the germ cell origin of pluripotent ESCs. (A) The inner cell mass cells of the blastocyst are positive for Oct $3 / 4$ and Sox 2 expression. During further development, primordial germ cell (PGC) specification in mouse implantation embryos (blastocyst $(\sim$ E3.5) stage onwards) is marked by the expression of germ cell markers Blimp 1 and Fragilis followed by reactivation of Oct $3 / 4$ and Sox 2 and is completed by $\sim \mathrm{E} 6.5-\mathrm{E} 7.5$ in vivo. This period of in vivo PGC specification parallels with the in vitro ESCs generation from pre-implantation blastocysts ( $\sim$ E3.5) together with 3-5 days of ESCs outgrowth. (B) On the other hand, reprogramming of somatic cells to iPS cells using the four Yamanaka factors (Oct3/4, Sox2, KIf4 and c-Myc) leads to the early appearance of germ cell markers followed by the activation of endogenous Oct $3 / 4$ and Sox2 and subsequent establishment of pluripotent state. This pattern of gene activation is equivalent to that of PGC specification and ESC establishment as discussed above. (C) The active chromatin state of germ cell markers in ESCs might indicate the developmental origin of ESCs from PGCs; the presence of bivalent chromatin at the promoter regions of pre-meiotic genes indicate the poised state for germ line commitment.

doi:10.1371/journal.pone.0022413.g006 
and $\mathrm{H} 3 \mathrm{~K} 9 \mathrm{ac})$ and the repressive $(\mathrm{H} 3 \mathrm{~K} 27 \mathrm{me} 3)$ histone modifications, which is a hallmark of key developmental regulation/lineage specific genes $[32,33]$. The observed active chromatin state at GC marker genes might indicate the possible early germ cell specification epigenetic marks in pluripotent cells. Conversely, the bivalent chromatin state at PrM marker genes might represent the poised germ cell lineage specification or the heterogeneous expression of these genes in pluripotent cells.

Recent advances in direct reprogramming of somatic cells to induced pluripotency opened new avenues not only for tailormade patient-specific cells for future regenerative medicine in addition to advancing our knowledge of the basic biology of establishment and maintenance of pluripotency [34]. Of particular interest is the role of GC/PrM markers during iPS cell generation using the four Yamanaka's factors. We analyzed the activation of GC/PrM markers along with the endogenous activation of core pluripotency markers during somatic reprogramming and found the activation of the PGC specification markers Blimp1, Stella and Fragilis to occur much earlier (between day 6 and day 9 of reprogramming) than activation of the endogenous pluripotency markers Oct $3 / 4$ and Sox 2 (by day 12 of reprogramming). In contrast, the expression of the PrM markers Dazl, MVH and Stra 8 was only detectable by day 22 and in established iPS cell lines, respectively. Recent studies of the molecular mechanisms underlying somatic reprogramming revealed that somatic cells undergo mesenchymal to epithelial transition (MET) during early reprogramming to acquire pluripotency through BMP signaling and vital expression of E-cadherin [35,36]. Interestingly, during embryonic development, PGC precursors rely on inductive BMP signals followed by MET activation and Fragilis, Blimpl, Stella and E-cadherin expression [37,38]. Loss of BMP signals, Blimpl and E-cadherin expression results in the depletion or a reduced number of PGCs $[38,39,40,41]$. Taken together, we assume that even somatic cells acquire a "temporary" PGC/GC fate and finally establish pluripotency during reprogramming.

Based on our study and earlier reports [9], we propose a working model for the germ-cell origin of ESCs and the possible acquisition of PGC/GC fate by somatic cells during iPSCs generation (Fig. 6). According to our model, the ICM of blastocyst stage embryos ( $\sim$ E3.5) expresses key pluripotency markers Oct3/4, Sox2, and c-Myc. Following embryonic development, PGC specification in-vivo is marked by the expression of key PGC genes, where Blimpl is activated by BMP signaling [10], facilitates the activation of Stella and Ecadherin, initiates the repression of the somatic program, and reactivates the pluripotency network before PGCs acquire migratory properties [38] (Fig. 6A). Considering the GC fate and lineage commitment of PGCs, key germ-cell markers may have active chromatin, whereas PrM genes may show bivalent chromatin (Fig. 6C). Similarly, ESC generation also starts with isolation of $\sim \mathrm{E} 3.5$ blastocysts followed by culture to obtain outgrowth from the ICM. It is more likely that during the in-vitro ICM outgrowth, ICM cells proceed with the pre-programmed developmental program of PGC specification via BMP signals, initiate MET, begin expressing Fragilis, Blimpl, and Stella, reactivate pluripotency genes, and acquire the unique self-renewal property (Fig. 6A). The observed active chromatin state of Blimp1, Stella, and Fragilis thus might indicate the unique expression or PGC/GC origin of ESC and the bivalent chromatin state of Dazl and $M V H$ confers the germ-cell lineage commitment, as has been observed for other lineages (Fig. 6C). Similarly, during somatic reprogramming, addition of Oct3/4, Sox2, c-Myc, and Klf4 to somatic cells might mimic the in-vivo $\sim$ E3.5 blastocyst ICM cells and follows the induction of BMP signaling and hence the activation of Fragilis, Blimpl, Stella, and E-cadherin, and MET (Fig. 6B). Further, activation of the endogenous pluripotency network from the host cell genome finally establishes pluripotent cell characteristics (Fig. 6B). Finally, the chromatin state of GC/ PrM markers may also reflect their transition through germ-cell fate (Fig. 6C).

In summary, we show the expression of GC/PrM markers in all analyzed pluripotent cell types and show parallel but independent maintenance of GC/PrM networks from pluripotent networks. Through our data, we propose a hypothetical model for possible germ-cell origin of ESCs and suggest the plausible transition of somatic cells through germ-cell fate to achieve pluripotency. Further genetic and epigenetic studies aimed at PGC specification during ICM outgrowth may resolve and increase our knowledge of pluripotency.

\section{Supporting Information}

Figure S1 Effect of the downregulation of Oct3/4 in ES cells. (A) Real time qPCR demonstrating the down-regulation of $O c t 3 / 4$ and the expression profile of other pluripotency markers (K7f4, Nanog, Zfp206 and Lin28). (B) Expression profile of germ cell (Stella and Fragilis) and pre-meiotic (Dazl, MVH and Stra ${ }^{8}$ ) markers in $0 c t 3 / 4$ down-regulated ESCs. The dotted lines indicate the normalized expression levels of analyzed genes in control siRNA treated cells. The qPCR data of two biological replicates (including three technical replicates each) were calculated and represented as a mean \pm SD. Expression levels, which are statistically significant, are indicated with asterisks $\left({ }^{* * 0 * 1 *} \mathrm{p}<0.001\right)$. (TIF)

Figure S2 Epigenetic signature of pluripotency and GC/ PrM genes in ES cells. (A) Chip-seq data from the database showing that the promoter regions (red box) of pluripotency marker gene $O c t 3 / 4$ and germ cell markers BlimpI (PrdmI). Stella (Dppa3) and Fragilis (Ifitm3) representing open chromatin with abundance of active histone modification $\mathrm{H} 3 \mathrm{~K} 4 \mathrm{me} 3$ (green peaks) and are depleted of repressive marks like $\mathrm{H} 3 \mathrm{~K} 27 \mathrm{me} 3$ and H3K 9 me3 (highlighted with red and brown peaks respectively). In contrast, the promoter regions of pre-meiotic genes $M V H$ (Ddxf), Dazl, Hoxall and Pax5 were marked with both active and repressive histone modification marks, signifying their bivalent chromatin structure $(\mathbf{B})$. (TIF)

Figure S3 Histopathological analysis identifies iPS cellderived tumors as teratomas. Tumors grown in $\mathrm{RAG}^{-1-}$ $\mathrm{c} \gamma \mathrm{c}^{-1-}$ mice after injection of iPS cell lines \#xu2 and \#xu6 were HE stained. The tumors are teratomas showing ectodermal mesodermal and endodermal differentiations (* skin epithelium, \# cartilage, $\rightarrow$ muscle, gut epithelium). The scale bar represents $100 \mu \mathrm{m}$. (TIF)

Table S1 Primers used in RT-PCR.

(DOC)

Table S2 Quantitative real-time PCR primers for siRNA down regulation study.

(DOC)

Table S3 Quantitative real-time PCR primers used to test endogenous gene expression.

(DOC)

Table S4 List of antibodies used in Western blotting. (DOC) 
Table S5 Quantitative real-time PCR primers used in Chip assay.

(DOC)

\section{Acknowledgments}

The authors would like to thank Lukasz Smorag and Marieke Wolf for their support and valuable discussions. The authors would like to thank MSC distribution center, Texas A\&M health science center for providing mouse MSCs, Prof. Shinya Yamanaka for kindly providing retroviral

\section{References}

1. Evans MJ, Kaufman MH (1981) Establishment in culture of pluripotential cells from mouse embryos. Nature 292: 154-156.

2. Martin GR (1981) Isolation of a pluripotent cell line from early mouse embryos cultured in medium conditioned by teratocarcinoma stem cells. Proc Natl Acac Sci U S A 78: 7634-7638.

3. Matsui Y, Zsebo K, Hogan BL (1992) Derivation of pluripotential embryonic stem cells from murine primordial germ cells in culture. Cell 70: 841-847.

4. Resnick JL, Bixler LS, Cheng L, Donovan PJ (1992) Long-term proliferation of mouse primordial germ cells in culture. Nature 359: 550-551.

5. Kahan BW, Ephrussi B (1970) Developmental potentialities of clonal in vitro cultures of mouse testicular teratoma. J Natl Cancer Inst 44: 1015-1036.

6. Guan K, Nayernia K, Maier LS, Wagner S, Dressel R, et al. (2006) Pluripotency of spermatogonial stem cells from adult mouse testis. Nature 440: 1199-1203.

7. Kanatsu-Shinohara M, Inoue K, Lee J, Yoshimoto M, Ogonuki N, et al. (2004) Generation of pluripotent stem cells from neonatal mouse testis. Cell 119 1001-1012.

8. Takahashi K, Yamanaka S (2006) Induction of pluripotent stem cells from mouse embryonic and adult fibroblast cultures by defined factors. Cell 126: 663-676

9. Zwaka TP, Thomson JA (2005) A germ cell origin of embryonic stem cells? Development 132: 227-233.

10. Ohinata Y, Payer B, O'Carroll D, Ancelin K, Ono Y, et al. (2005) Blimpl is a critical determinant of the germ cell lineage in mice. Nature 436: 207-213.

11. Saiti D, Lacham-Kaplan O (2007) Mouse Germ Cell Development in-vivo and in-vitro. Biomark Insights 2: 241-252.

12. Saitou M, Barton SC, Surani MA (2002) A molecular programme for the specification of germ cell fate in mice. Nature 418: 293-300.

13. Sato M, Kimura T, Kurokawa K, Fujita Y, Abe K, et al. (2002) Identification of PGC7, a new gene expressed specifically in preimplantation embryos and germ PGC7, a new gene expressed
cells. Mech Dev 113: 91-94.

14. Bowles J, Koopman P (2007) Retinoic acid, meiosis and germ cell fate in mammals. Development 134: 3401-3411.

15. Geijsen N, Horoschak M, Kim K, Gribnau J, Eggan K, et al. (2004) Derivation of embryonic germ cells and male gametes from embryonic stem cells. Nature 427: 148-154.

16. Mise N, Fuchikami T, Sugimoto M, Kobayakawa S, Ike F, et al. $(2008$ Differences and similarities in the developmental status of embryo-derived stem cells and primordial germ cells revealed by global expression profiling. Genes Cells 13: 863-877.

17. Qing T, Shi $Y$, Qin H, Ye X, Wei W, et al. (2007) Induction of oocyte-like cells from mouse embryonic stem cells by co-culture with ovarian granulosa cells. from mouse embryonic stem
Differentiation 75: 902-911.

18. Zechner U, Nolte J, Wolf M, Shirneshan K, Haji NE, et al. (2009) Comparative methylation profiles and telomerase biology of mouse multipotent adult germline stem cells and embryonic stem cells. Mol Hum Reprod 15: 345-353.

19. Voss AK, Thomas T, Gruss $P$ (1997) Germ line chimeras from female ES cells. Exp Cell Res 230: 45-49.

20. Wernig M, Meissner A, Foreman R, Brambrink T, Ku M, et al. (2007) In vitro reprogramming of fibroblasts into a pluripotent ES-cell-like state. Nature 448: 318-324.

21. Okita K, Ichisaka T, Yamanaka S (2007) Generation of germline-competent induced pluripotent stem cells. Nature 448: 313-317. constructs for iPS cells generation and Dr. Michael Engelke for helping in retroviral transduction experiments.

\section{Author Contributions}

Conceived and designed the experiments: XX DVKP WE. Performed the experiments: XX SL XT TK JN RD. Analyzed the data: DVKP RD UZ. Contributed reagents/materials/analysis tools: WE. Wrote the paper: XX DVKP UZ

22. Chazaud C, Yamanaka Y, Pawson T, Rossant J (2006) Early lineage segregation setween epiblast and primitive endoderm in mouse blastocysts through the Grb2-MAPK pathway, Dev Cell 10: 615-624.

23. Khromov T, Krishna Pantakani DV, Nolte J, Wolf M, Dressel R, et al. (2010 Global and gene-specific histone modification profiles of mouse multipotent adult germline stem cells. Mol Hum Reprod.

24. Dressel R, Guan K, Nolte J, Elsner L, Monecke S, et al. (2009) Multipotent adult germ-line stem cells, like other pluripotent stem cells, can be killed by cytotoxic T lymphocytes despite low expression of major histocompatibility complex class 1 molecules. Biol Direct 4: 31 .

25. Dressel R, Nolte J, Elsner L, Novota P, Guan K, et al. (2010) Pluripotent stem cells are highly susceptible targets for syngeneic, allogeneic, and xenogeneic cells are highly susceptible targets for sy

26. Mikkelsen TS, Ku M, Jaffe DB, Jssac B, Licberman E, et al. (2007) Genomewide maps of chromatin state in pluripotent and lineage-committed cells. Nature wide maps of $553-560$.

27. Nayernia K, Lee JH, Drusenheimer N, Nolte J, Wulf G, et al. (2006) Derivation of male germ cells from bone marrow stem cells. Lab Invest 86: $654-663$

28. Carter MG, Sharov AA, VanBuren V, Dudekula DB, Carmack CE, et al. (2005) Transcript copy number estimation using a mouse whole-genome oligonucleotide microarray. Genome Biol 6: R61.

29. Efroni S, Duttagupta R, Cheng J, Dehghani H, Hocppner DJ, et al. (2008) Global transcription in pluripotent embryonic stem cells. Cell Stem Cell 2 437-447.

30. Meshorer E, Misteli T (2006) Chromatin in pluripotent embryonic stem cells and differentiation. Nat Rev Mol Cell Biol 7: 540-546.

31. Roeder RG (2005) Transeriptional regulation and the role of diverse coactivators in animal cells. FEBS Lett 579: 909-915.

32. Azuara V, Perry P, Sauer S, Spivakov M, Jorgensen HF, et al. (2006) Chromatin signatures of pluripotent cell lines. Nat Cell Biol 8: 532-538.

33. Bernstein E, Duncan EM, Masui O, Gil J, Heard E, et al. (2006) Mouse polycomb proteins bind differentially to methylated histone H3 and RNA and are enriched in facultative heterochromatin. Mol Cell Biol 26: 2560-2569.

34. Cox JL, Rizzino A (2010) Induced pluripotent stem cells: what lies beyond the paradigm shift. Exp Biol Med (Maywood) 235: 148-158.

35. Li R, Liang J, Ni S, Zhou T, Qing X, et al. (2010) A mesenchymal-to-epithelial transition initiates and is required for the nuclear reprogramming of mouse fibroblasts. Cell Stem Cell 7: 51-63.

36. Samavarchi-Tehrani P, Golipour A, David L, Sung HK, Beyer TA, et al. (2010 Functional genomics reveals a BMP-driven mesenchymal-to-epithelial transition in the initiation of somatic cell reprogramming. Cell Stem Cell 7:64-77.

37. Matsui Y (2009) The molecular mechanisms regulating germ cell development and potential. J Androl 31: 61-65.

38. Saitou M (2009) Germ cell specification in mice. Curr Opin Genet Dev 19: $386-395$

39. Lawson KA, Dunn NR, Roclen BA, Zeinstra LM, Davis AM, et al. (1999) Bmp4 is required for the generation of primordial germ cells in the mouse embryo. Genes Dev 13: 424 436.

40. Okamura D, Kimura T, Nakano T, Matsui Y (2003) Cadherin-mediated cell interaction regulates germ cell determination in mice. Development 130: 6423-6430.

41. Ying Y, Liu XM, Marble A, Lawson KA, Zhao GQ (2000) Requirement of Bmp8b for the generation of primordial germ cells in the mouse. Mol Endocrinol 14: 1053-1063. 


\subsection{Dppa3 binds to the IG-DMR of the Dlk1-Dio3 imprinting cluster and prevents its imprinting loss during iPS cell generation.}

The observation that the expression of GC marker genes precedes the expression of pluripotency maker genes during generation of iPSCs prompted us to study their role in generation of authentic iPSCs with greater efficiencies. iPSCs are generated by the forced expression of a set of pluripotency-related transcription factors. However, the majority of mouse iPS cell lines displayed an imprinting defect at the Dlk1-Dio3 imprinting cluster and fails to show germ line transmission. The normal expression level of Gtl2, a noncoding RNA from the Dlk1-Dio3 cluster, is considered as a bona fide marker for authentic induced pluripotency. In the second part of this thesis, we studied the role of GC marker genes (Blimp1, Ifitm3 and Dppa3) in establishment of authentic iPSCs and found that supplementation of Dppa3 to the classical reprogramming factor cocktail can enhance the reprogramming and prevent the Dlk1-Dio3 imprinting defect. At the molecular level, we show that Dppa3, the epigenetic regulator, binds to a specific region within the intergenic-differentially methylated region (IG-DMR), the imprinting control region of the Dlk1-Dio3 cluster. Further studies showed that the binding of Dppa3 can lead to a reduced binding of Dnmt3a, a de novo DNA methyltransferase, to the IG-DMR locus thereby preventing the abnormal methylation observed in iPSCs. Taken together, our results demonstrate that Dppa3-mediated epigenetic regulation is essential for generating authentic iPSCs.

Authors: Xingbo Xu, Toshinobu Nakamura, Xiaoying Tan, Lukasz Smorag, Ralf Dressel, Antje Fitzner, Matthias Linke, Jessica Nolte, Ulrich Zechner, Wolfgang Engel and D.V. Krishna Pantakani

Status: Submitted to Nature Genetics journal

\section{Author contributions to the work:}

1. Xingbo $\mathrm{Xu}$ : Conception and design of experiments, data collection, data analysis, data interpretation, helped in drafting the manuscript.

2. Toshinobu Nakamura: Data collection, data analysis, data interpretation.

3. Lukasz Smorag: Conception, data collection, data analysis.

4. Ralf Dressel: Performed teratoma formation assay, data analysis.

5. Antje Fitzner: Data collection, data analysis.

6. Matthias Linke: Data collection, data analysis.

7. Jessica Nolte: Conception and design of experiments, data analysis. 
8. Ulrich Zechner: Performed DNA methylation assay, data analysis, data interpretation, helped in drafting the manuscript.

9. Wolfgang Engel: Conception and design of experiments, financial support, helped in drafting the manuscript.

10. D.V. Krishna Pantakani: Conception and design of experiments, data analysis, data interpretation, experiment coordination, drafted the manuscript. 


\title{
Dppa3 binds to the IG-DMR of the Dlk1-Dio3 imprinting cluster and prevents its imprinting loss during iPS cell generation
}

\author{
Xingbo Xu ${ }^{1}$, Toshinobu Nakamura ${ }^{2}$, Lukasz Smorag ${ }^{1}$, Ralf Dressel ${ }^{3}$, Antje Fitzner ${ }^{4}$, Matthias Linke ${ }^{4}$, Jessica \\ Nolte $^{1}$, Ulrich Zechner ${ }^{4}$, Wolfgang Engel ${ }^{1}$ and D.V. Krishna Pantakani ${ }^{1+}$ \\ 'Institute of Human Genetics, University of Goettingen, Goettingen, Germany. ${ }^{2}$ Department of Pathology, Graduate School of Medicine, \\ Osaka University, Osaka, Japan. ${ }^{3}$ Department of Cellular and Molecular Immunology, University of Goettingen, Goettingen, Germany. \\ ${ }^{4}$ Institute of Human Genetics, Johannes Gutenberg-University Mainz, Mainz, Germany
}

\$Correspondence: D. V. Krishna Pantakani, Tel: +49-551-399302; Fax: +49-551-399303; E-mail: krishna.ifl@gmail.com

\begin{abstract}
Reprogramming of somatic cells into induced pluripotent stem cells (iPSCs) often results in defects at the genetic and epigenetic level. Of particular interest is the loss of imprinting at the paternally imprinted Dlk1-Dio3 cluster in a majority of the iPSC clones, which ultimately fail to show full pluripotent cell characteristics. In the present study, we analyzed the role of germ cell (GC) marker genes (Blimp1, Fragilis, and Dppa3), that have been described to become activated early during somatic cell reprogramming, in establishment of authentic pluripotency. We found that Dppa3, the guardian of the maternal genome and imprinted loci that protects them against DNA demethylation during early embryonic development, is present only in iPSC lines showing expression of Gt12 (Gtl2on), an indicator of normal imprinting at Dlk1-Dio3 cluster, but not in iPSCs with loss of imprinting (Gtl2off). Subsequently, we found that exogenous Dppa3 together with classical reprogramming factors can efficiently reprogram somatic cells into iPSC clones that all display normal imprinting at Dlk1-Dio3 region. To dissect the mechanism by which Dppa3 can establish/maintain imprints at the Dlk1-Dio3 cluster, we performed chromatin immunoprecipitation assays with a Dppa3 antibody on embryonic stem cell (ESC) chromatin and found Dppa3 to be associated with a specific region within the intergenic differentially methylated region (IG-DMR) of Dlk1-Dio3. Further, the forced expression of Dppa3 during reprogramming lead to the maintenance of imprinting at this cluster by counteracting and reducing the binding of Dnmt $3 \mathrm{a}$, a de novo DNA methyltransferase that establishes DNA methylation. Interestingly, these findings are in parallel with a recent report which describes that the addition of vitamin $\mathrm{C}$ can enhance the reprogramming and correct the Dlk1-Dio3 imprinting defect through the inhibition of Dnmt3a binding to this region. Collectively, our results show that Dppa3 is a genetic factor necessary for preventing abnormal imprinting at the Dlk1-Dio3 region during somatic cell reprogramming.
\end{abstract}

Keywords: iPSCs, Dppa3, Dnmt3a, Gt12, IG-DMR, Dlk1-Dio3, imprinting, Vitamin C.

\section{Introduction}

Pluripotent stem cells boast the differentiation potential virtually into any cell type of the body, and hence hold a great promise for regenerative medicine applications ${ }^{1}$. Recent advances in reprogramming strategies unveiled the induction of pluripotency in somatic cells by using few transcription factors resulting in the generation of induced pluripotent stem cells (iPSCs) $)^{2,3}$. These iPSCs are morphologically and functionally similar to ESCs, 
although they frequently show minor differences in gene expression, DNA methylation, imprinting of the Dlkl-Dio3 cluster and gene copy number-7. The transcriptome analysis of iPSCs in comparison to ESCs revealed that the expression of coding and non-coding genes encoded by the Dlkl-Dio3 imprinting cluster is misregulated due to the aberrant acquisition of DNA methylation at the maternal allele along with the normally methylated paternal allele $^{7}$. The iPSCs with defects in genomic imprinting are developmentally compromised and show reduced chimerism after injection into blastocysts and no germline transmission ${ }^{7,8}$. Recently, addition of vitamin $\mathrm{C}(\mathrm{Vc})$ during reprogramming was shown to result in iPSCs with normal Dlkl-Dio3 imprinting, yet the factor expressed in a Vc-dependent manner was not identified ${ }^{9}$.

Genomic imprinting is an epigenetic phenomenon established during gametogenesis and involves differential DNA methylation and post-translational histone modifications. Short DNA sequences called imprinting control regions (ICRs) are methylated on either the maternal or paternal allele to regulate expression of the imprinted gene in $c i{ }^{10}$. These marks lead to exclusive or preferential parent-specific monoallelic expression of imprinted genes $^{11,12}$. During embryonic development a pool of primordial germ cells (PGCs) gives rise to progenitors of adult gametes. After their specification and upon their arrival at the genital ridge, these PGCs undergo demethylation of the whole genome including an erasure of parent-specific methylation marks of imprinted genes, the so-called imprints ${ }^{13}$. The reestablishment of genomic imprinting in germ cells according to the sex of the embryo is initiated after the entry of PGCs into gonads and continues through germ cell differentiation ${ }^{14,15}$.

The imprint acquisition according to the sex of the embryo is regulated by several components such as primary sequence specificity, chromatin configuration, non-histone proteins and transcriptional events (reviewed by Arnaud, $2010^{16}$ ).
Specifically, the de novo DNA methyltransferase Dnmt3a and its related protein Dnmt3l, which has no methyltransferase activity, were shown to be essential for imprint establishment at several imprinted loci ${ }^{17-19}$. In addition to the DNA methylation machinery, several other DNA binding proteins such as Zfp57, Nlrp2, Nlrp7, Ctcfl and Prmt 7 are implicated in the establishment of imprints in a sex-specific manner ${ }^{20-24}$. Once established in germ cells, several factors are known to faithfully maintain and transmit the imprints during the early stages of embryogenesis to all somatic lineages (reviewed by Arnaud, $2010^{16}$ ). Dppa3/PGC7 is one such factor expressed mainly in germ cells and known to protect some of the maternal as well as paternal imprints during the wave of DNA demethylation occurring in early embryogenesis ${ }^{25}$.

Previously, we have shown that germ cell (GC) marker genes such as Blimp1, Fragilis and Dppa3 are expressed in all pluripotent cell types and emerge early during somatic cell reprogramming into ${ }_{i P S C s}{ }^{26}$. In the present study, we evaluated whether these GC marker genes, in particular Dppa3, play any role in the maintenance of Dlkl-Dio3 imprinting during the generation of iPSCs. Interestingly, we found the expression of Blimpl and Fragilis in iPSCs with normal as well as defective Dlkl-Dio3 imprinting, whereas Dppa3 was expressed only in cells having normal Dlkl-Dio3 imprinting. We then reprogrammed MEFs in the presence of Dppa3, in addition to Yamanaka's reprogramming factors (OSKM), and found that all iPSC colonies show normal imprinting at the Dlk1-Dio3 region. These iPSCs were morphologically and functionally similar to ESCs and displayed all characteristics of pluripotent cells including germline transmission. At the molecular level, we observed that Dppa3 is associated with the intergenic differentially methylated region (IG-DMR) at the Dlkl-Dio3 region which counteracts the binding of Dnmt3a binding to this region during reprogramming. Further, we found that vitamin C can activate the Dppa3 
expression, which lets us suggest that Dppa3 is the genetic component essential for the proper establishment/maintenance of Dlk1-Dio3 imprinting during iPSC generation.

\section{Materials and methods}

\section{Cell culture}

Mouse ESCs and iPSCs were cultured in Dulbecco's Modified Eagle Medium (DMEM) (PAN, Germany) supplemented with $15 \%$ fetal calf serum (FCS) (PAN, Germany), 2mM L-glutamine (PAN, Germany), $50 \mu \mathrm{M} \quad \beta$-mercaptoethanol (Gibco/Life Technology, Germany), $1 \%$ non-essential amino acids (NEAA) (Gibco/Life Technologies, Germany), $1 \%$ sodium pyruvate (Gibco/Life Technologies, Germany), $1 \%$ penicillin/streptomycin (PAN, Germany) and $1000 \mathrm{U} / \mathrm{ml}$ leukemia inhibitory factor (LIF) (Millipore, Germany). For reprogramming studies with low serum medium, the StemPro medium (Invitrogen) supplemented with StemPro supplement (Invitrogen), $\quad 1 \% \quad$ FCS, $2 \mathrm{mM}$ L-Glutamine, $1 \mathrm{mM}$ Sodium pyruvate, $1 \%$ NEAA, $100 \mu \mathrm{M} \beta$-mercaptoethanol, and $1000 \mathrm{U} / \mathrm{ml} \mathrm{LIF}$ was used either in the presence or absence of $50 \mu \mathrm{g} / \mathrm{ml}$ vitamin C (Sigma).

\section{Construction of expression vectors}

For generating a retroviral expression vector of Fragilis, the ORF was PCR-amplified from cDNA of mouse ESCs and cloned into the pGEM-T Easy vector (Life Technologies, Germany). After verification by sequencing, the Fragilis ORF was cloned into EcoRI restriction sites of pMXs-hOct $3 / 4^{3}$ by replacing hOct $3 / 4$. For generating the pMXs-Blimp1 expression vector, the EcoRI and XhoI fragment of HA-tagged Blimpl from pMSCV-HA-Blimp1-IRES-GFP ${ }^{27}$ was subcloned into EcoRI and XhoI restriction sites of pMXs-hOct $3 / 4^{3}$ by replacing hOct $3 / 4$. The expression of each construct was verified by transfection into Plat-E cells followed by Western blot analysis. The constructs pMXs-hOct $3 / 4$ and pMXs-Dppa3 were obtained from Addgene.

\section{Generation of iPS cells}

We used retroviral expression vectors for Oct $3 / 4$, Sox 2 , Klf4, and c-Myc ${ }^{2}$ together with that of either Fragilis, Dppa3, or Blimp1 to reprogram MEFs into iPSCs. Briefly, MEFs isolated from transgenic Nanog-EGFP mice ${ }^{28}$ were transduced with retroviral particles as described ${ }^{2}$. To establish iPS cell lines, colonies which appeared after 8-12 days of transduction were picked and cultured in 24-well plates with standard ESC culture conditions and were monitored for the ESC-like morphology using an Olympus-IX71 inverted microscope (Olympus, Germany). Further, several independent iPSC lines were established from each combination to examine the expression of $G t / 2$ as well as for further characterization. Alternatively, iPSCs were generated in low serum medium as described above.

\section{Immunostaining and alkaline phosphatase staining}

Immunostaining was performed as described previously $^{29}$ using mouse monoclonal antibodies to SSEA1 (ab16285, Abcam). Cytochemical staining for alkaline phosphatase activity was performed using Leukocyte Alkaline Phosphatase Kit (Sigma-Aldrich).

\section{Protein extraction and Western blotting}

Total protein extracts were prepared using lysis buffer (10mM Tris- $\mathrm{HCl}$ (pH 8.0), 1mM EDTA, $2.5 \%$ SDS, $100 \mathrm{mM}$ PMSF) containing protease inhibitor cocktail (Roche). Protein samples were resolved on 4-12\% SDS-PAGE (Life Technologies, Germany) and transferred onto nitrocellulose membrane (Amersham Biosciences). Membranes were processed using standard Western blot protocols, and signals were detected using a chemiluminescent kit (Santa Cruz Biotechnology). 
Antibody sources are listed in supplementary tables (Supplementary Table S1).

\section{Total RNA extraction and quantitative real time} PCR analysis

Total RNA was extracted from cells using NucleoSpin miRNA kit (Machery-Nagel, Germany) following the manufacturer's protocols. For the mRNA quantification experiment, $5 \mu \mathrm{g}$ of total RNA was converted into cDNA using the SuperScript II system (Life sciences, Germany). For the miRNA quantification assay, $1 \mu \mathrm{g}$ of total RNA was used for cDNA synthesis using miScript II RT Kit (Qiagen, Germany). For qPCR analysis, diluted cDNA (1/10) was used as a template in QuantiFast SYBR Green (Qiagen, Germany) based method and run on ABI7900HT Real-Time PCR System (Applied Biosystems, Germany). The qRT-PCR data were normalized to housekeeping genes (Hprt and Gapdh) and represented as percentage of expression of housekeeping genes. Primers used in RT-PCR and qPCR are listed in Supplementary Table S2.

\section{Genomic DNA isolation and methylation analysis of the $G t / 2$ IG-DMR}

Genomic DNA isolation from cells and bisulfite pyrosequencing were performed as previously described $^{30}$. Primers used for analysis of the Gtl2 IG-DMR are listed in Supplementary Table S2.

\section{Chromatin immunoprecipitation assay}

Chromatin immunoprecipitation (ChIP) assays were performed on ESCs and MEFs undergoing reprogramming using the Diagenode OneDay ChIP kit. Briefly, the cells $(3 \times 106$ cell per pull down) were cross-linked using formaldehyde and lysed with the shearing kit (Diagenode, Belgium) followed by sonication with a bioruptor (Diagenode, UCD-200 TM) to obtain an average chromatin size of $400 \mathrm{bp}$. Then, the sheared chromatin was immunoprecipitated with $5 \mu \mathrm{g}$ of antibodies as indicated and the Diagenode OneDay ChIP protocol was used to extract the chromatin bound to each specific antibody. Rabbit IgG was used as a control in mock ChIP experiments. Quantitative analysis was performed using real-time qPCR and the primers listed in supplementary table S2. Ct values were determined for both immunoprecipitated DNA and a known amount of DNA from the input sample for different primer pairs. The amount of immunoprecipitated chromatin corresponding to a particular genomic locus was then calculated from the qPCR data using the delta-delta $\mathrm{Ct}$ method (ddCt) and reported as fold enrichment relative to the background (IgG). All fold enrichment values are means of three independent experiments.

\section{Teratoma formation assay}

The teratoma formation assay was performed as previously described ${ }^{31,32}$. Briefly, iPS cells $\left(1 \times 10^{6}\right.$ cells) were injected subcutaneously into RAG2 $2^{-/} \mathrm{c} \gamma \mathrm{c}^{-/-}$ mice lacking $\mathrm{T}, \mathrm{B}$, and natural killer (NK) cells. Tumor growth was monitored weekly by palpation and size was recorded using linear calipers. Animals were sacrificed when a tumor diameter of $1 \mathrm{~cm}$ was reached. Autopsies were performed and tumor tissue was placed in phosphate-buffered $4 \%$ formalin for 16 $\mathrm{h}$ and then embedded in paraffin. For histological analysis, the specimens were stained with hematoxylin and eosin (HE).

\section{Statistical Analysis}

All qPCR data for RNA expression analysis (two or more biological replicates) were calculated using the standard curve method. The GraphPad Prism 4.0 software was used to calculate the statistical significance.

\section{Results}

\section{Dppa3 expression positively correlates with the activation of $\mathrm{Gtl} 2$ in iPS cells}

The loss of Dlk1-Dio3 imprinting was reported to be a frequently occurring phenomenon during 
A.

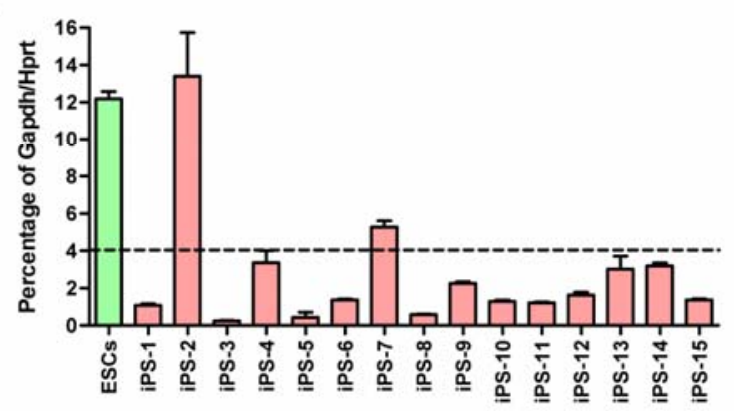

B.

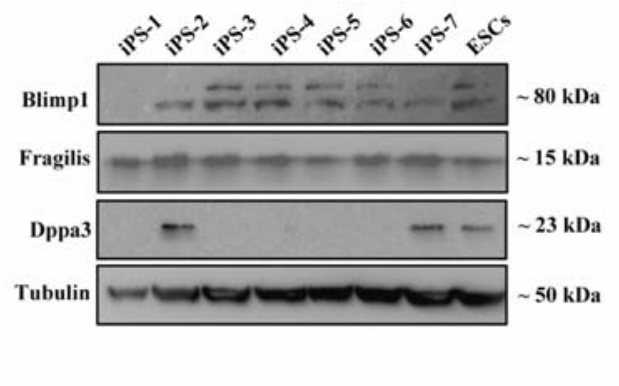

C.

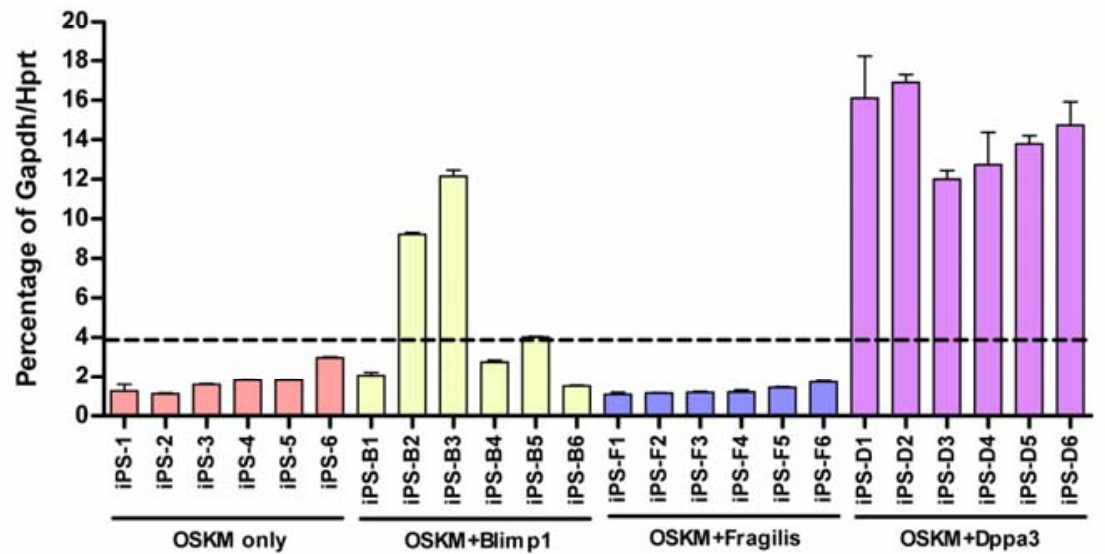

D.

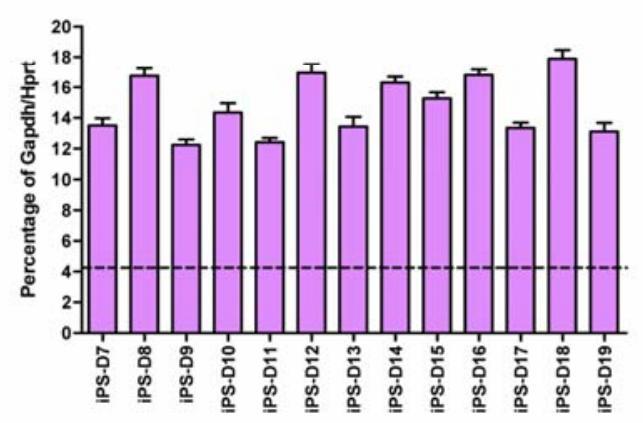

E.

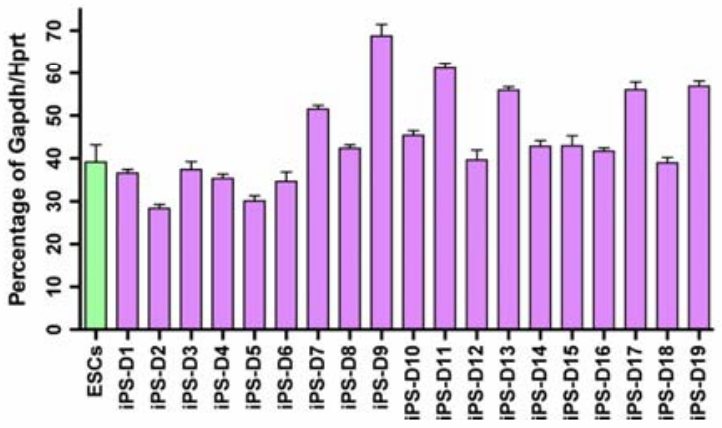

Figure 1. Expression of Dppa3 positively correlates with proper expression of imprinted genes of from the Dlk1-Dio3 cluster in iPSCs. (A) qRT-PCR data showing the expression of Gtl2 above the threshold (dotted line ${ }^{9}$ ) only in 2/15 iPSCs generated using classical Yamanaka factors (OSKM). The expression of Gtl2 in ESCs was used as a control. (B) Western blot analysis for Blimp1, Fragilis, and Dppa3 expression in iPSCs generated using OSKM. The protein lysate derived from ESCs was used as a control. (C) Gtl2 expression analysis in iPSCs generated using either OSKM or OSKM in combination with indicated germ cell marker genes (Blimp1 (B), Fragilis (F), and Dppa3 (D)). (D) qRT-PCR data showing the expression of Gtl2 in additional iPSC clones generated using OSKM+D. (D) qRT-PCR data showing the expression of Dlkl in iPSC clones generated using OSKM+D. The expression of Dlkl in ESCs was used as a control. 
A.

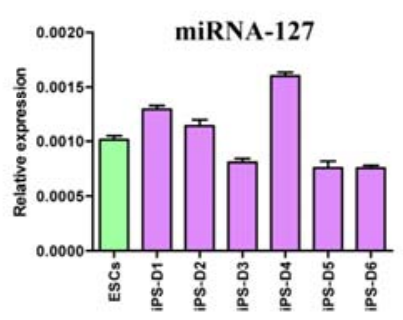

D.

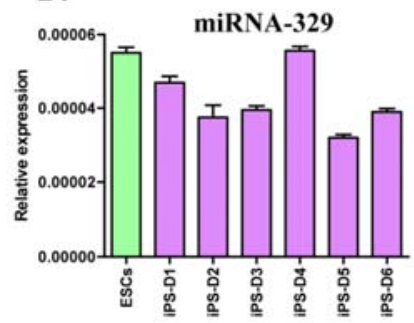

B.

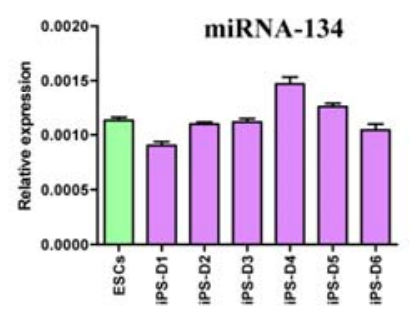

C.

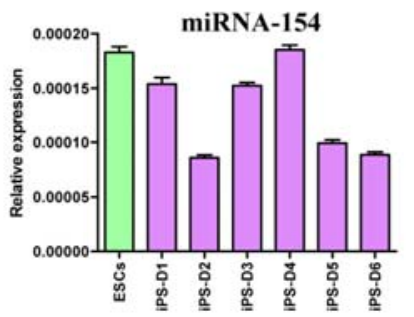

E.

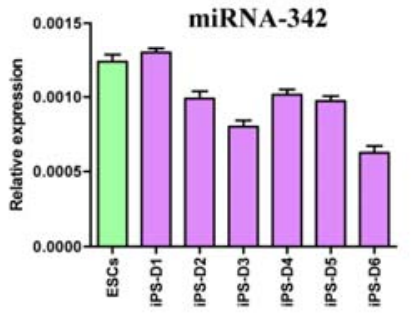

F.

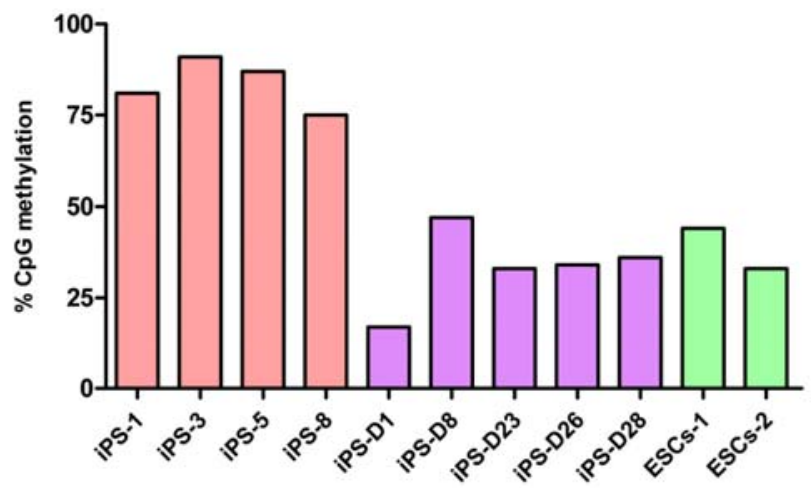

Figure 2. Expression of miRNAs encoded by the Dlk1-Dio3 cluster and methylation of the Gtl2 IG-DMR in iPSCs generated using Dppa3. (A-C), qRT-PCR data showing the expression of miRNA-127 (A), miRNA-134 (B), miRNA-154 (C), and miRNA-329 (D) in OSKM+D iPSCs. cDNA derived from ESCs was used as a control for miRNA expression. (E) DNA methylation analysis of the Gtl2 IG-DMR (4 CpG sites) in OSKM+D iPSCs and OSKM only Gtl2 ${ }^{\text {off }}$ iPSCs; genomic DNA from ESCs was used as a control.

mouse iPSC generation ${ }^{7}$. The iPSCs with aberrant imprinting at the Dlkl-Dio3 cluster failed to generate chimeras with germline transmission, one of the central characteristic of pluripotent cells ${ }^{7,8}$. Since then, the activation/expression of $G t / 2$, a maternally expressed non-coding RNA of this imprinting cluster is used as a marker to identify the authentic iPSC colonies. To decipher the underlying cause of aberrant imprinting, we established several iPSCs lines from mouse embryonic fibroblasts (MEFs) using the classical Yamanaka approach. We found only 2/15 clones (iPS-2 and iPS-7) having Gtl2 expression $\left(G t l 2^{\text {on }}\right)$ above the threshold level ${ }^{9}$ (Fig. 1A), further indicating that the defect at Dlkl-Dio3 imprinting cluster is a common outcome during somatic cell reprogramming. Recently, we have 
A.

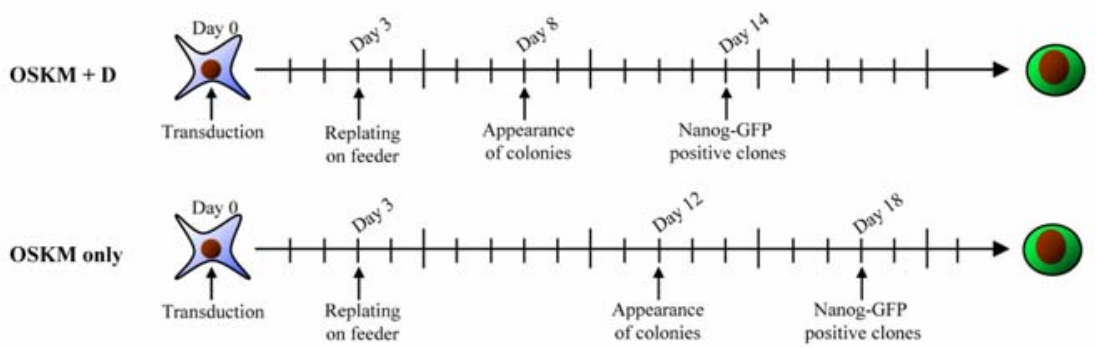

B.
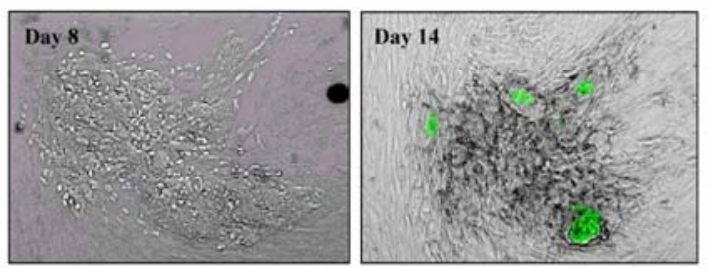

D.

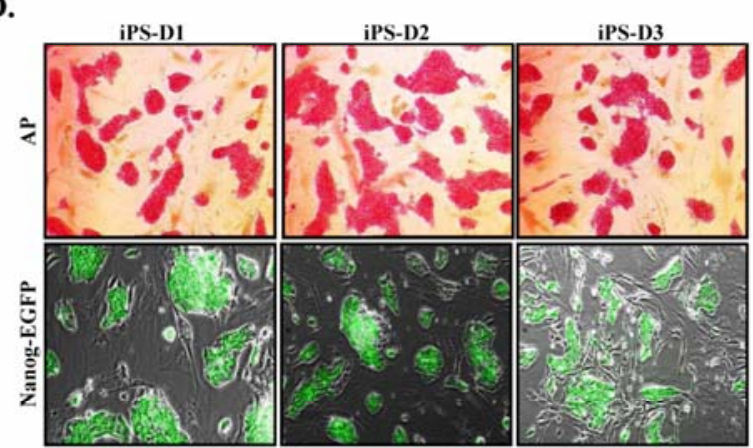

C.

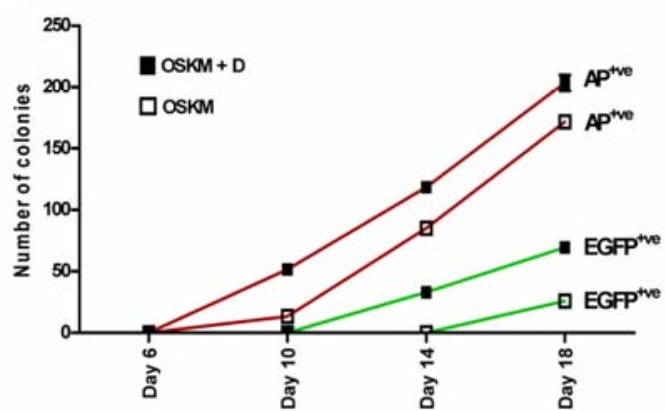

F.
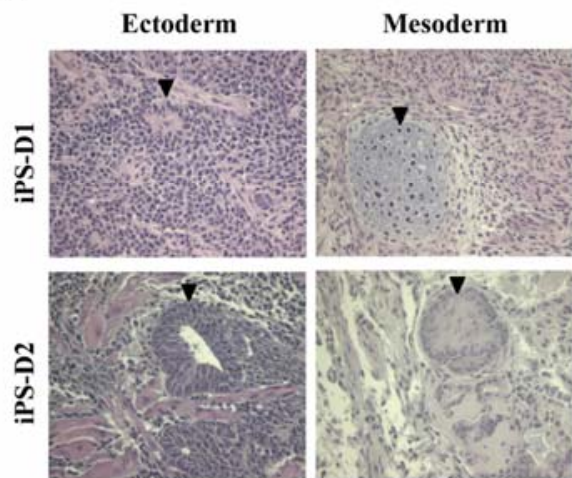

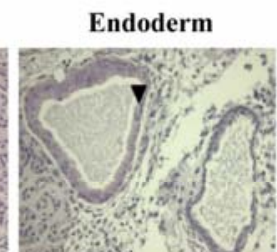

G.
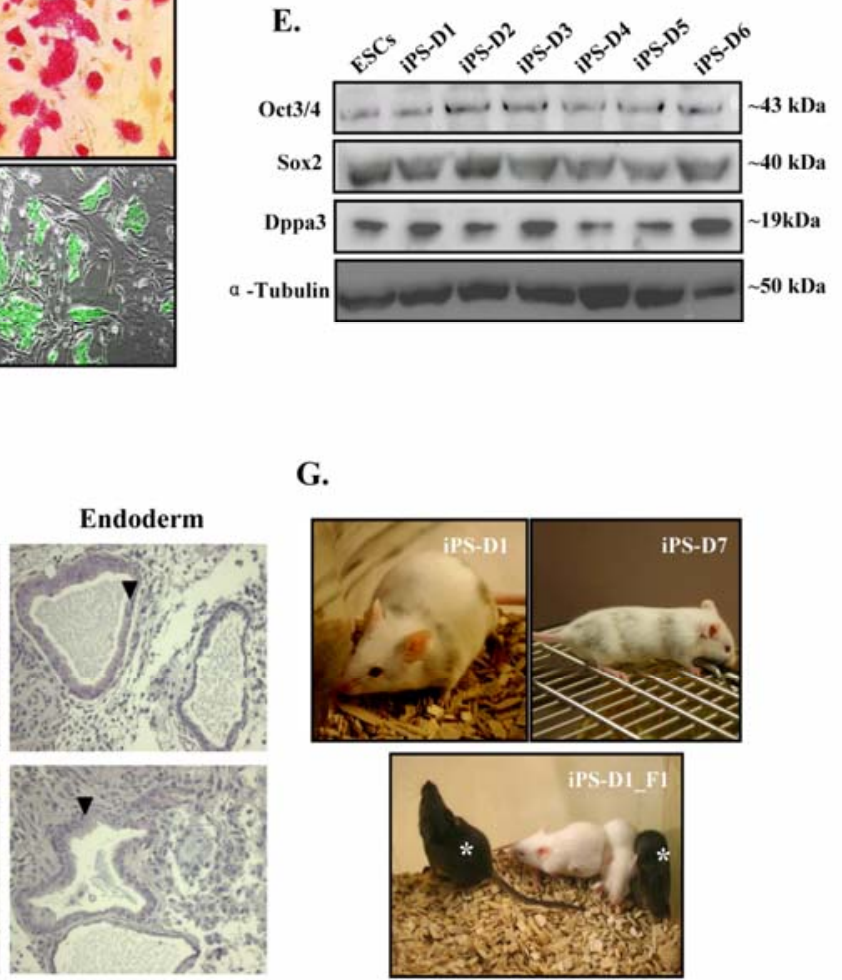

Figure 3. Dppa3 enhances the reprogramming and generates fully pluripotential iPSCs. (A) Time scale showing the start of viral transduction followed by appearance of ESC-like and Nanog-EGFP-positive colonies during iPSCs generation using either OSKM or OSKM in combination with Dppa3 (OSKM+D). (B) Bright-field image showing the appearance of ESC-like colonies already by day 8 of reprogramming using 
OSKM+D (left panel). The same colony was positive for Nanog-driven EGFP at day 14 (right panel). (C) Line graph showing the number of AP-positive $\left(\mathrm{AP}^{+v e}\right)$ and EGFP-positive (EGFP ${ }^{+v e}$ ) colonies during the reprogramming time-course in the presence of OSKM (open box) or OSKM+D (closed box). (D) iPSC colonies (iPS-D) generated using OSKM+D show AP activity (top panel) and activate the pluripotency reporter Nanog-EGFP (bottom panel). (E) Western blots showing the expression of pluripotency marker genes Oct $3 / 4$ and Sox2 as well as Dppa3 in OSKM+D iPSCs (iPS-D). Protein extracts from ESCs were used as a control. (F) Images showing the presence of cell type derivatives of all three germ layers in teratomas obtained from OSKM+D iPSCs. Arrowheads indicate the presence of the representative tissue/cell type from the respective germ layer. (G) Images showing the chimeras obtained from OSKM+D iPSCs (upper panel) and their germline transmission (lower panel). Asterisks indicate the pups derived from the germline competent OSKM+D iPSCs.

shown that the expression of germ cell (GC) marker genes such as Blimp1, Fragilis and Dppa3 precedes the expression of endogenous pluripotency-related genes, such as Oct3/4, Sox2, Nanog and Klf4, during somatic cell reprogramming ${ }^{26}$. To identify whether these GC marker genes have any role in establishment/maintenance of Dlk-Dio3 imprinting, we analyzed several iPSC colonies for the expression of GC marker genes (Fig. 1B). The Western blot analysis for the expression of Blimp1 and Fragilis did not show any differences between $G t l 2^{\text {on }}$ and $G t l 2^{\text {off }}$ clones (Fig. 1A, B). However, the expression of Dppa3 was observed only in Gt12 ${ }^{\text {on }}$ clones but not in $\mathrm{Gtl}^{\text {off }}$ clones (Fig. 1A, B), indicating that the expression of Dppa3 might be crucial for the establishment/maintenance of Dlkl-Dio3 imprinting, at least during reprogramming.

To comparatively analyze the effect of GC marker gene expression on maintenance of Dlk1-Dio3 imprinting, we generated a Fragilis retroviral construct and verified its expression (Supplementary Fig. S1A), while the generation of Blimp1 and Dppa3 constructs was described previously ${ }^{2,27}$. The transduction of MEFs with each of the GC markers (Blimp1, Fragilis, and Dppa3 abbreviated as B, F, and D, respectively) along with Yamanaka factors (Oct3/4, Sox2, Klf4, and c-Myc abbreviated as OSKM, respectively) resulted in the establishment of iPS colonies, which are morphologically similar to ESCs and positive for alkaline phosphatase (ALP) activity and expressed
Nanog promoter-driven EGFP (data not shown). The Gtl2 expression analysis in 6 individual iPSC colonies from each combination of transductions revealed that the addition of Dppa3 to the OSKM results in all $G t l 2^{\text {on }}$ colonies (6/6), while Blimpl gave rise to only $3 / 6 \mathrm{Gt} / 2^{\text {on }}$ colonies (Fig. 1C). Further, 6/6 iPS colonies generated with either OSKM alone or OSKM supplemented with Fragilis showed Gtl2 expression levels below the threshold limit (Fig. 1C).

To confirm the faithful maintenance of imprinting at Dlkl-Dio3 cluster by Dppa3 (D) supplementation, we next generated additional iPS clones with OSKM+D and found all of them to express Gtl2 above the threshold level (Fig. 1D). We checked the expression of $D l k l$, the paternally expressed gene in this imprinting cluster, and found its levels comparable to those in ESCs (Fig. 1E). The Dlkl-Dio3 imprinting cluster is also known to harbor several maternally expressed miRNAs which are underexpressed in $G t l 2^{\text {off }}$ clones $^{7}$. Hence, we analyzed the expression of Dlkl-Dio3 cluster-encoded miR-127, miR-134, miR-154, miR-329, and miR-342 in OSKM+D iPSCs and found them to be expressed in all iPSCs (Fig. 2A-E). Then, we verified if the presence and absence of Gtl2 mRNA in OSKM+D iPSCs and OSKM Gtl $2^{\text {off }}$ iPSCs, respectively, is associated with differences in DNA methylation levels at the Gtl2 IG-DMR, the ICR of the Dlkl-Dio3 imprinting cluster. Indeed, we detected a moderate hypomethylation of the IG-DMR 
A.

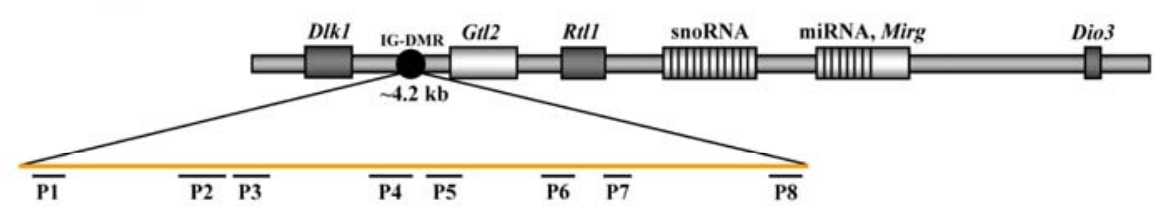

B.

C.
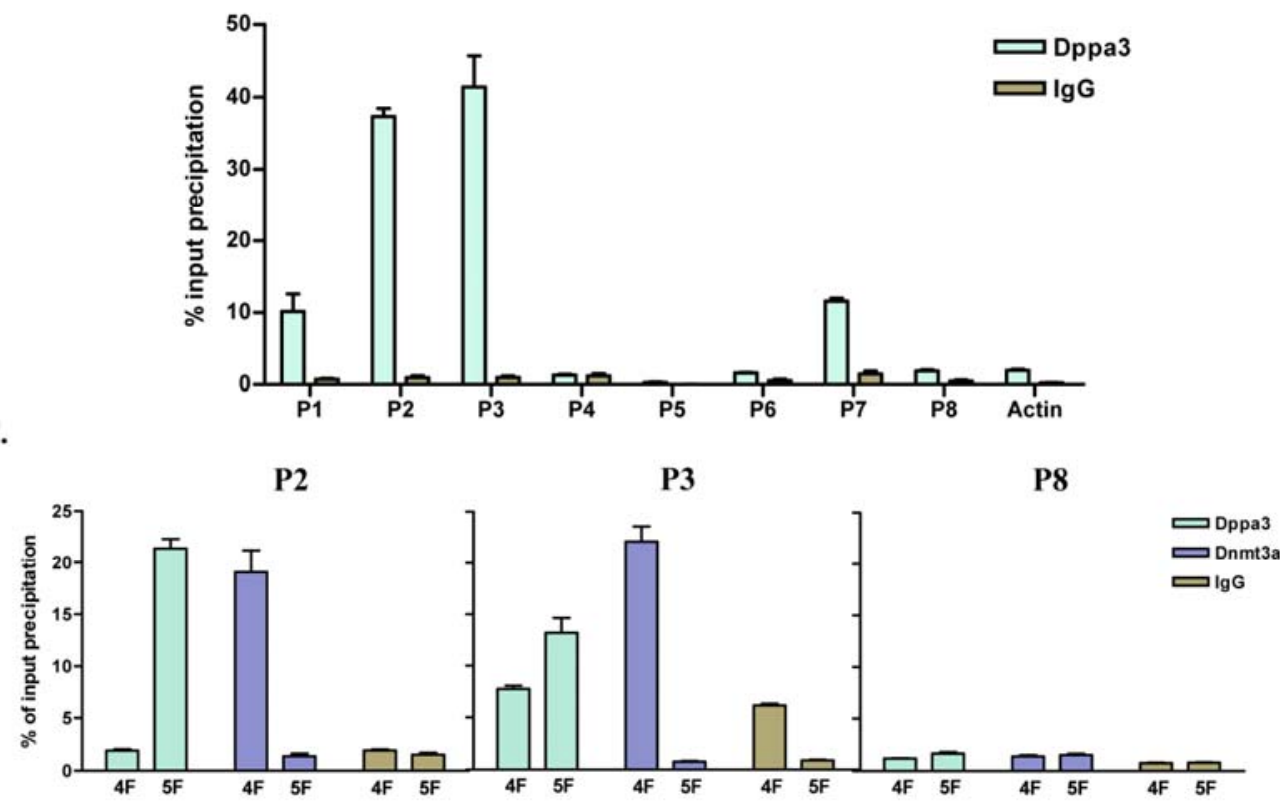

D.

P2

P3

P8

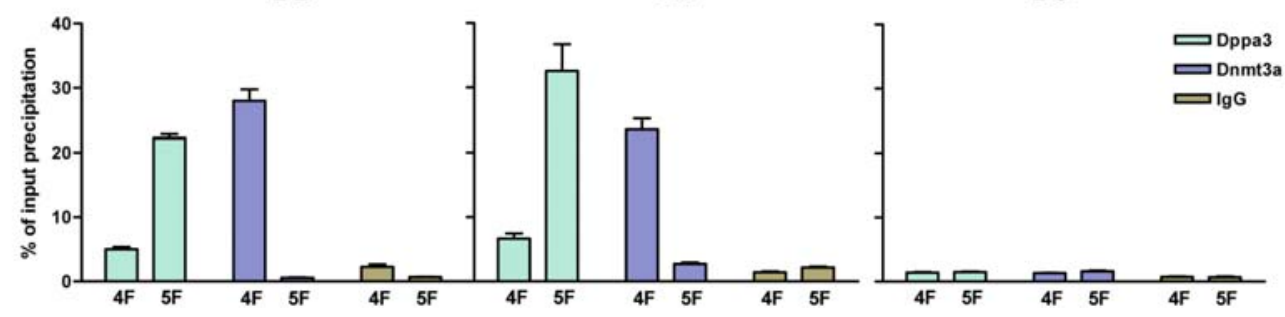

Figure 4. Dppa3 binds to a specific region within the IG-DMR of the Dlk1-Dio3 imprinting cluster. (A) Simplified schematic diagram showing the Dlk1-Dio3 imprinting cluster along with paternally expressed genes (dark grey boxes) and maternally expressed genes (light grey boxes). The imprinting control region, intergenic differentially methylated region (IG-DMR) (black circle), that spans $\sim 4.2 \mathrm{~kb}$, and the 8 PCR amplicons (P1-P8) covering the entire IG-DMR are indicated. (B) Chromatin immunoprecipitation (ChIP) followed by qPCR analysis for Dppa3 (cyan) binding sites across the 8 PCR amplicons of the IG-DMR in ESCs. ChIP with IgG (olive) was used as a control. (C) ChIP followed by qPCR analysis for Dppa3 (cyan) and Dnmt3a (blue) binding at the P2, P3 and P8 amplicons of the IG-DMR in OSKM (4F) and OSKM+D (5F) at 9 days of reprogramming. ChIP with IgG (olive) was used as a control. (D) ChIP followed by qPCR analysis for Dppa3 (cyan) and 
Dnmt3a (blue) binding at the P2, P3 and P8 amplicons of the IG-DMR in OSKM (4F) and OSKM+D (5F) at 12 days of reprogramming. ChIP with IgG (olive) was used as a control.

in all analyzed OSKM $+\mathrm{D}$ iPSCs compared to a hypermethylation of the IG-DMR in all analyzed OSKM Gtl2 ${ }^{\text {off }}$ iPSCs (Fig. 2F). Collectively, these results suggest that Dppa3 expression can prevent aberrant hypermethylation of the IG-DMR and Dlkl-Dio3 imprinting defects during MEF reprogramming.

Dppa3 enhances the reprogramming and contributes to the generation of all authentic iPSC colonies

During the course of reprogramming, the OSKM+D-transduced MEFs appeared as early colonies already by day- 8 in contrast to the OSKM-only-transduced cells, which showed colony morphology only by day 12 (Fig. 3A, B). Moreover, OSKM+D-reprogrammed cells activated the Nanog-EGFP by day 14; whereas OSKM-treated cells were EGFP-positive only at around day 18 of the reprogramming (Fig. 3A, B). Addition of Dppa3 to the reprogramming factors greatly enhanced the number of AP- and EGFP-positive iPSC colonies (Fig. 3C). We characterized several iPSC clones derived from Dppa3-treated cells (iPS-D) for the expression of ALP, Nanog-EGFP and pluripotency marker genes such as Oct $3 / 4$ and Sox 2 as well as Dppa3. The studied iPS-D clones were positive for all analyzed pluripotent cell characteristics (Fig. 3D, E). To test the teratoma formation ability of iPS-D cells, we injected two different iPSC clones (iPS-D1 and iPS-D2) into immunodeficient mice and found teratoma consisting of all three germ-layer derivatives, confirming the pluripotency of the two iPSC clones (Fig. 3F). Furthermore, the injection of iPS-D clones into blastocysts resulted in chimeras (Fig. 3G) and chimeras generated from iPS-D1 were tested for their germline transmission ability. Offspring from iPS-D1 were obtained (Fig. 3G) and a genotyping PCR confirmed the presence of OSKM+D constructs (Supplementary Fig. S2A).

Next, we investigated whether Dppa3 can replace any of the reprogramming factors (OSKM) during somatic cell reprogramming. MEFs reprogrammed in the absence of each of the reprogramming factors but instead supplemented with Dppa3 failed to generate any AP-positive colonies by day 18 of reprogramming (data not shown). Although we obtained few smaller colonies in the absence of c-Myc, it could not be due to Dppa3 as it was already reported that c-Myc is dispensable for the reprogramming process ${ }^{33}$. These results suggest that Dppa3 works in concert with other reprogramming factors but might not be able to drive the reprogramming process without any of these factors.

\section{Dppa3 physically interacts with the IG-DMR of the Dlk1-Dio3 cluster}

Previously, Dppa3 was shown to be essential for protecting maternal chromosomes against DNA demethylation during early post-fertilization development ${ }^{25}$. It was also reported that Dppa3 protects against DNA demethylation at several but not all imprinted genes/regions ${ }^{25}$. To test the hypothesis that Dppa3 is physically associated with the IG-DMR of the Dlkl-Dio3 region, we performed a chromatin immunoprecipitation (ChIP) assay with an antibody against Dppa3 on ESC chromatin. Since the IG-DMR of the Dlk1-Dio3 cluster spans around $4.2 \mathrm{~kb}$, we designed 8 PCR amplicons (P1-P8) covering the entire IG-DMR region (Fig. 4A). The qPCR analysis of immunoprecipitated chromatin revealed that Dppa3 binds specifically to the IG-DMR region represented by PCR amplicons P2 and P3 PCR (Fig. 4B). Recent data showed that Dnmt3a is highly enriched at the IG-DMR during somatic cell reprogramming, probably leading to the 
A.

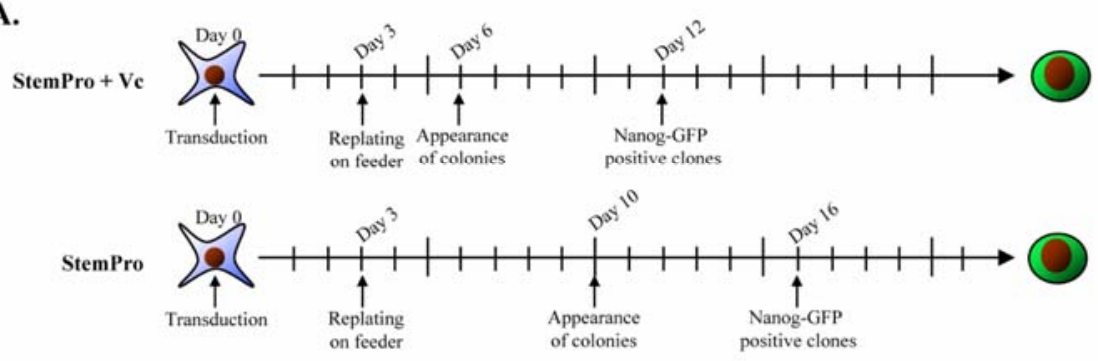

B.

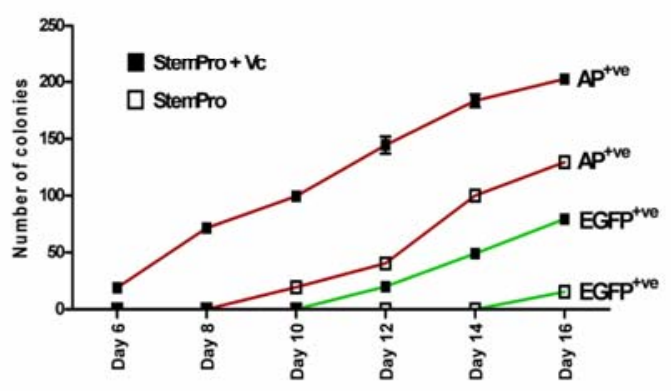

D.

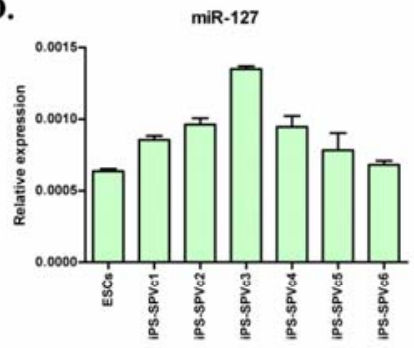

C.

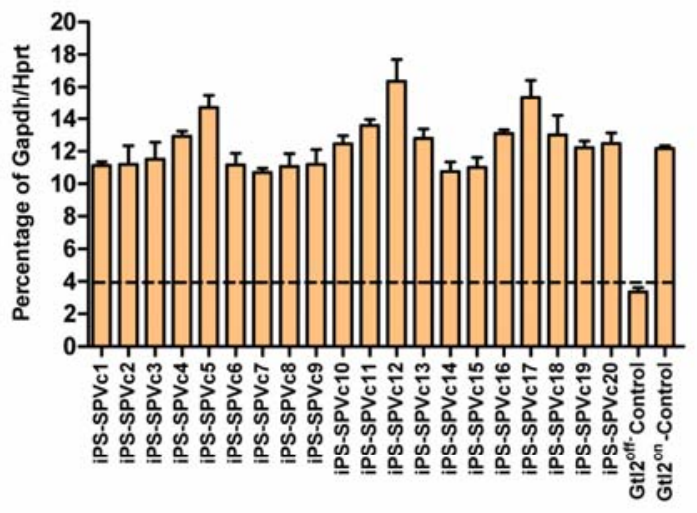

E.
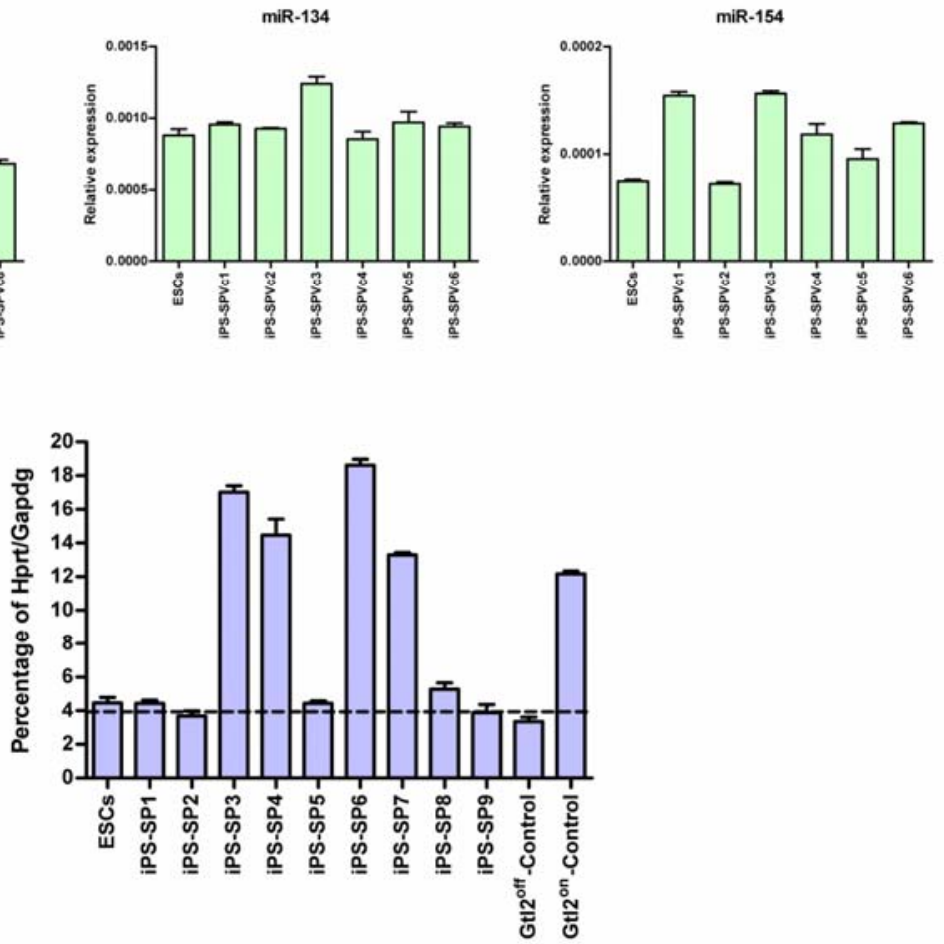

Figure 5. Vitamin $\mathrm{C}$ enhances the reprogramming and generates fully pluripotential iPSCs in low serum medium. (A) Time scale showing the start of viral transduction followed by appearance of ESC-like and Nanog-EGFP-positive colonies during iPSC generation using OSKM in StemPro (low serum medium) supplemented with $\mathrm{Vc}(\mathrm{SPV})$ or StemPro without $\mathrm{Vc}(\mathrm{SP})$. (B) Line graph showing the number of $\mathrm{AP}^{+\mathrm{ve}}$ and 
EGFP $^{+v e}$ colonies during the reprogramming time course in the presence of SP (open box) or SPVc (closed box). (C) qPCR data showing the expression of Gtl2 in iPSC clones generated using StemPro+Vc (iPS-SPVc). Previously generated $G t / 2^{\text {off }}$ and $G t l 2^{\text {on }}$ iPSC clones were used as controls. (D) qRT-PCR data showing the expression of miRNA-127 (left panel), miRNA-134 (middle panel), and miRNA-154 (right panel) in iPS-SPVc clones. ESCs were used as a control for miRNA expression. (E) qPCR data showing the expression of Gtl2 in iPSC clones generated using SP only (iPS-SP). Previously generated $G t l 2^{\text {off }}$ and $G t l 2^{\text {on }} i P S C$ clones with $G t l 2^{\text {off }}$ and $G t l 2^{\text {on }}$ were again used as controls.

silencing of the maternal allele and that this effect can be counteracted by the addition of ascorbic acid (vitamin $\mathrm{C}, \mathrm{Vc})^{9}$. This led us to analyze whether exogenous Dppa3 can also counteract Dnmt3a binding at the IG-DMR. Interestingly, the association of Dnmt3a with the IG-DMR PCR amplicons P2 and P3 was significantly reduced in OSKM+D-transduced cells at day-9 of reprogramming, whereas OSKM only-transduced cells showed enrichment for Dnmt3a at this region (Fig. 4C). Similar results were obtained at day-12 of reprogramming (Fig. 4D), confirming that Dppa3 protects the IG-DMR of the Dlkl-Dio3 locus against DNA hypermethylation by counteracting Dnmt3a binding.

These results prompted us to evaluate the expression of Gtl2 and Dlkl in Dppa3-deficient $\mathrm{ESCs}^{25}$. qRT-PCR results indicated no obvious differences in expression levels of Glt2 and Dlk1 between Dppa3-deficient and control ESCs (data not shown). Further, DNA methylation analysis of the Dlk1-Dio3 IG-DMR revealed no significant differences between Dppa3-deficient and control ESCs, whereas the DNA methylation of Rasgrfl, another paternally methylated locus, showed hypomethylation in Dppa3-knockout cells (Supplementary Fig. S3).

Modified-StemPro medium enhances the reprogramming and results in the generation of exclusively $\mathrm{Gtl}^{\text {on }}$ iPSCs

During the course of our study, we also investigated whether StemPro-based low-serum medium (SPVc), which we regularly use for the culture of spermatogonial stem cells $(\mathrm{SSCs})^{34}$, can generate all $G t l 2^{\text {on }}$ iPSCs with greater efficiency. To this end, we reprogrammed MEFs with OSKM in SPVc medium and found ESC-like colonies already by day 6 of reprogramming which finally resulted in a 2-fold increase in AP- and Nanog-EGFP-positive colonies (Fig. 5A and B). Subsequently, we checked the expression of Gtl2 in several iPSC lines, which were generated using SPVc medium (iPSC-SPVc), and found all of them to be Gtl2 ${ }^{\text {on }}$ iPSCs (Fig. 5C). Further, to validate the activation of Dlk1-Dio3 locus, we have analyzed the expression of three maternally expressed miRNAs (miR-127, -134, -154) and identified them to be expressed in all analyzed iPSC-SPVc (Fig. 5D). These results led us to hypothesize that the components of SPVc help in proper maintenance of imprinting at Dlkl-Dio3 locus during reprogramming. Our SPVc medium inherently contains vitamin $\mathrm{C}(\mathrm{Vc})$ that was reported to protect against the los of Dlk1-Dio3 imprinting during reprogramming 9 . Hence, we asked whether StemPro-based low-serum medium depleted from Vc (SP) can also generate exclusively $G t / 2^{\text {on }}$ iPSCs with similar reprogramming kinetics like SPVc. However, the use of SP medium resulted in slower reprogramming kinetics and only 4 of the 9 generated iPSC clones had the Gt12 expression markedly above the threshold (Fig. 5B and 5E). Our results are in line with observations of Stadtfeld et al. (2012) ${ }^{9}$, indicating that $\mathrm{Vc}$ can not only enhance the reprogramming but also prevent the loss of Dlk1-Dio3 imprinting. 
A.

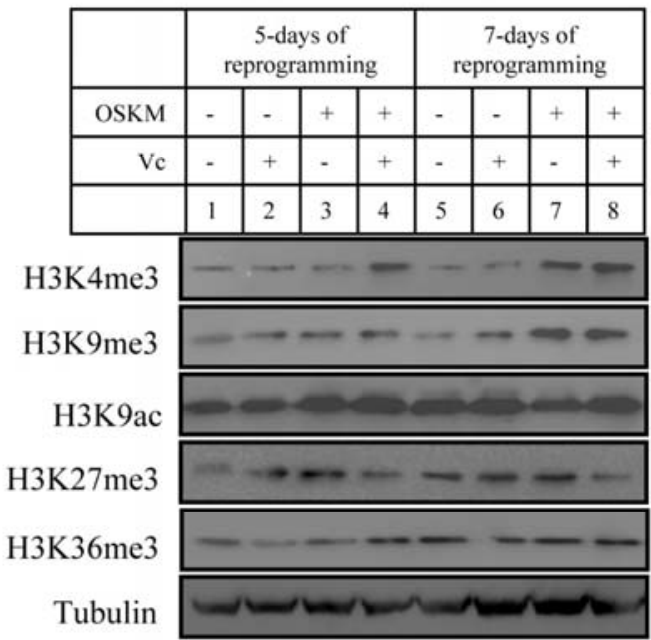

B.
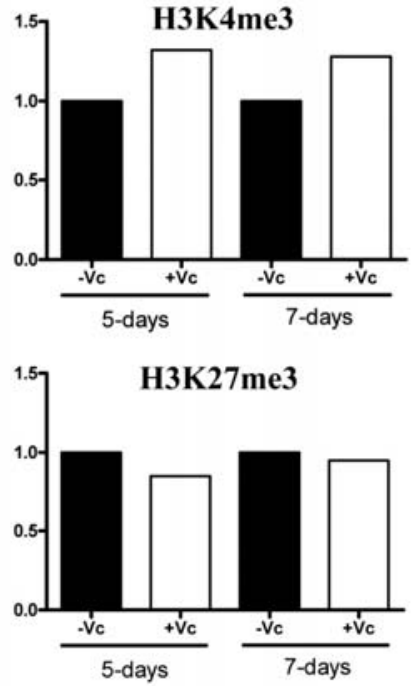

C.

Oct3/4

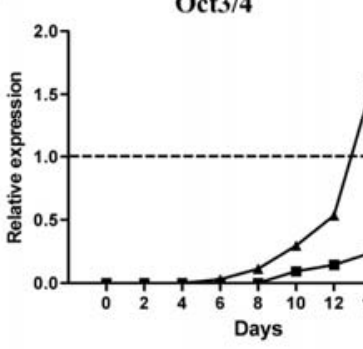

Sox2

Dppa3

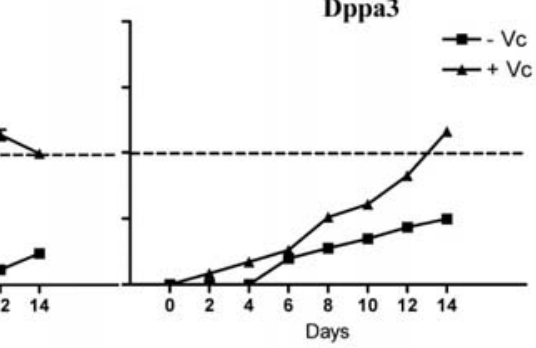

D.

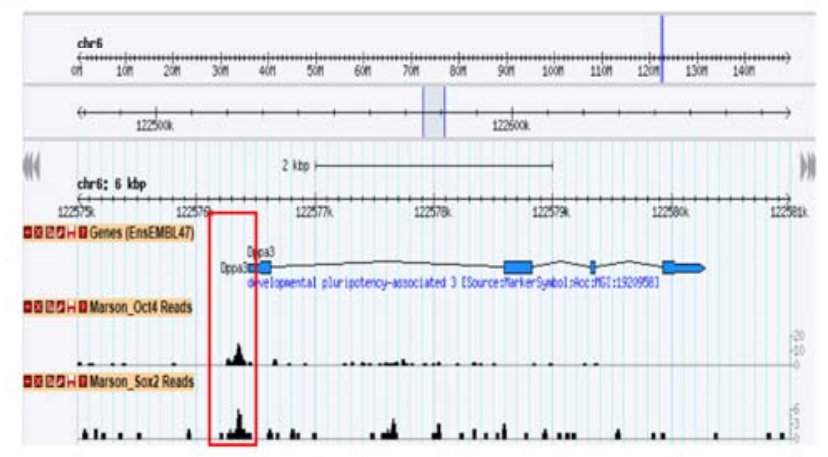

E.

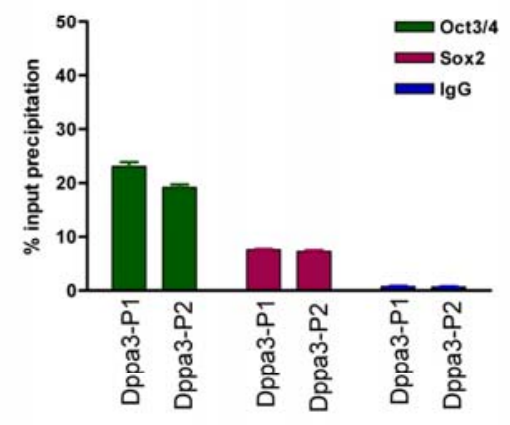

Figure 6. Vitamin $C$ mediates the global chromatin relaxation and early-activation of pluripotency-related genes. (A) Western blots showing the expression of various histone modifications during OSKM reprogramming ( 5 days and 7 days) in standard ESC culture medium and either presence or absence of Vc (+ or -, respectively). MEFs supplemented with no OSKM and Vc (lanes 1 and 5) or either Vc only (lanes 2 and 6) or OSKM only (lanes 3 and 7) were used as controls. All blots were reprobed with an anti-tubulin antibody and one representative blot is shown as a loading control. (B) Densitometric quantification of $\mathrm{H} 3 \mathrm{~K} 4 \mathrm{me} 3$ and $\mathrm{H} 3 \mathrm{~K} 27 \mathrm{me} 3$ band intensities at 5 days of reprogramming (lanes 3 and 4 in A) and 7 days of reprogramming (lanes 7 and 8 in A). (C) qPCR data showing the expression of Oct3/4 (left panel), Sox 2 (middle panel), and Dppa 3 (right panel) during the OSKM reprogramming time course in standard ESC culture medium 
and either presence (+Vc, closed box) or absence (-Vc, closed triangle) of Vc. (D) Publicly available ChIP-Seq data showing the Oct4 and Sox2 binding (black peaks) at the Dppa3 promoter region (red box). (E) ChIP followed by $\mathrm{qPCR}$ analysis for Oct $3 / 4$ (green) and Sox 2 (maroon) binding at the Dppa3 promoter region (Dppa3-P1 and Dppa3-P2) in ESCs. ChIP with IgG (blue) was used as a control.

\section{Vitamin C (Vc) mediates global chromatin relaxation leading to early-activation of Dppa3}

Recently, it was reported that the Vc-treatment results in global chromatin changes mostly in methylation levels of histone $\mathrm{H} 3$ lysine $36(\mathrm{H} 3 \mathrm{~K} 36)^{35}$. The observed molecular parallels between Dppa3 and $\mathrm{Vc}$ in protecting against the imprinting loss at the Dlkl-Dio3 cluster prompted us to investigate whether Vc treatment leads to the activation of Dppa3 during early stages of reprogramming. To answer this question we reprogrammed MEFs using OSKM in standard ESC medium supplemented either with or without $\mathrm{Vc}(+\mathrm{Vc}$ and $-\mathrm{Vc}$, respectively) and analyzed the cells for changes at various histone modification marks and expression levels of Oct3/4, Sox2, and Dppa3. After 5 days of reprogramming, MEFs treated with $\mathrm{Vc}(+\mathrm{Vc})$ showed enrichment for H3K4me3, a histone modification mark associated with active gene transcription, whereas MEFs treated without $\mathrm{Vc}(-\mathrm{Vc})$ showed basal levels of $\mathrm{H} 3 \mathrm{~K} 4 \mathrm{me} 3$ (Fig. 6A, B). In contrast to $\mathrm{H} 3 \mathrm{~K} 4 \mathrm{me} 3$, the levels of $\mathrm{H} 3 \mathrm{~K} 27 \mathrm{me} 3$, a repressive histone mark, were greatly reduced in $+\mathrm{Vc}$ cells but not in $-\mathrm{Vc}$ cells (Fig. 6A, B). These changes were also evident at day 7 of reprogramming (Fig. 6A, B). Expression of endogenous $O c t 3 / 4$ and Sox 2 during reprogramming was activated at least two days earlier in $+\mathrm{Vc}$ cells compared to $-\mathrm{Vc}$ cells (Fig. 6C). Further, by day 12 of reprogramming, we observed much higher expression levels of endogenous Oct3/4 and Sox 2 in $+\mathrm{Vc}$ cells compared to $-\mathrm{Vc}$ cells (Fig. 6C). Surprisingly, expression of Dppa3 was already detected by day 2 of reprogramming in $+\mathrm{Vc}$ treated MEFs, but only by day 6 of reprogramming in control (-Vc) cells (Fig. 6C).

To correlate the early activation of Dppa3 with chromatin changes induced by $\mathrm{Vc}$, we hypothesized that exogenous reprogramming factor(s) might gain access to the Dppa3 promoter and activate its expression early in Vc-treated cells. To verify this assumption, we checked the publicly available ChIP-Seq data of Oct $3 / 4$ and Sox 2 binding sites/targets across the ESC genome and found Oct $3 / 4$ and Sox 2 binding sites at the Dppa 3 promoter region (Fig. 6D). To validate these global ChIP-Seq data, we performed ChIP assays with Oct $3 / 4$ and Sox 2 antibodies and subsequently qRT-PCR for the Dppa 3 promoter region using two PCR amplicons. The qRT-PCR data clearly indicate that both Oct $3 / 4$ and Sox2 bind to Dppa3, although with varying affinities (Fig. 6E). Collectively, these results suggest that Vc facilitate the transcription of Dppa3 during early stages of reprogramming and this in turn leads to the binding of Dppa3 to the Dlkl-Dio3 cluster and restricts the access of Dnmt3a to this locus.

\section{Discussion}

Although the generation of iPSCs from somatic cells has became a routine and reproducible method, the fully pluripotential capacity of each iPSC line generated is in question. In support of this view, two independent research groups have identified an imprinting defect at the Dlkl-Dio3 cluster in the majority of mouse iPSC cell lines, which ultimately leads to the loss of germline transmission in chimeras $^{7,8}$. These results strongly suggested that not all generated iPSC lines are faithfully reprogrammed. Recently, we have shown that GC marker genes such as Blimp1, Fragilis, and Dppa3 are expressed earlier than the endogenous pluripotency-related genes and suggested that they might play a crucial role in establishment of pluripotency ${ }^{26}$. In the current study, we endeavored to find out the role of these GC marker genes in the establishment of authentic iPSC 
lines with proper imprinting at the Dlkl-Dio3 cluster. In our experiments, we made the interesting discovery that all iPSC lines with imprinting defects at the Dlk1-Dio3 cluster did not express the Dppa3 protein, whereas normally imprinted iPSC lines showed Dppa3 expression. These results indicate that the expression of Dppa3 might be essential for the imprint establishment/maintenance at the Dlk1-Dio3 cluster. In line with this hypothesis, all iPSC lines generated in the presence of exogenous Dppa3 showed no Dlk1-Dio3 imprinting defects. In detail, these iPSC lines displayed normal DNA methylation at the Gtl2 IG-DMR, expression of Gtl2 and miRNAs encoded within the Dlkl-Dio3 cluster as well as all pluripotent cell characteristics.

Dppa3 is highly expressed during embryonic development as well as in germ cells and pluripotent stem cells ${ }^{36,37}$. Gene ablation studies of Dppa3 in mice revealed that it functions during early embryonic development to protect the maternal genome against the first wave of active DNA demethylation $^{25}$. Moreover, Dppa3 was also shown to be essential for protecting several but not all imprinted loci and transposons against DNA demethylation during post-fertilization events ${ }^{25}$. Interestingly, the paternally methylated Dlkl-Dio3 region was not affected in Dppa3-null fertilized oocytes, whereas $H 19$ and Rasgrfl, the two other paternally methylated imprinted loci, showed partial loss of imprints ${ }^{25}$. Similarly, our current analysis of Dppa3-deficient ESCs also revealed no obvious defects in Dlk1-Dio3 imprinting. In contrast to these observations, the expression of Dppa3 during somatic cell reprogramming was found to be positively correlated with the activation of $G t l 2$, thus resulting in normal imprint establishment or maintenance at the Dlk1-Dio3 cluster in iPSCs. This discrepancy could be mainly due to artificial reprogramming of somatic cells versus normal early embryonic development. Another explanation would be that the imprinting of Dlkl-Dio3 is stably maintained on the sperm-derived paternal allele in
Dppa3-null embryos. In support of this view, the imprinting of the paternally methylated $H 19$ and Rasgrfl genes shows fluctuations in ESCs, whereas Dlk1-Dio3 displays stable imprinting in these cells.

Dppa3 is regarded as a DNA binding protein and indeed in-vitro studies have shown that it binds non-specifically to $\mathrm{DNA}^{25}$. A recent report described that Dppa3 binds to nucleosomes containing a dimethylation mark at lysine 9 of histone 3 $(\mathrm{H} 3 \mathrm{~K} 9 \mathrm{me} 2)^{38}$. Our ChIP analysis showed that Dppa3 binds to a specific region in the Dlk1-Dio3 IG-DMR, which also suggests that the interaction might involve modified histones. Unlike many imprinted loci, the IG-DMR of the Dlkl-Dio3 cluster was reported to contain less or no $\mathrm{H} 3 \mathrm{~K} 9 \mathrm{me} 2^{38}$. Thus, it can be hypothesized that the association of Dppa3 with the IG-DMR is mediated by histone modifications other than $\mathrm{H} 3 \mathrm{~K} 9 \mathrm{me} 2$ or some binding partner.

Recently, Stadtfeld et al., $(2012)^{9}$ showed that Vc can prevent the Dlk1-Dio3 imprinting defect during reprogramming mainly by preserving the active chromatin structure at this locus. The resulting active chromatin was suggested to counteract the recruitment of Dnmt3a, a de novo DNA methyltransferase, to this locus thus maintaining the normal imprinting in Vc-treated cells ${ }^{9}$. The identified role of Dppa3 in preventing the Dlkl-Dio3 imprinting defect suggests that Vc might function through the activation of Dppa3 or that both $\mathrm{Vc}$ and Dppa3 may function in a similar mechanistic pathway. This hypothesis is supported by the observation that Dppa3 transcripts are upregulated Vc-treated cells. Moreover, the acceleration and efficiency of reprogramming seen after addition of $\mathrm{Vc}^{9,35,39}$ can also be observed after addition of exogenous Dppa3, indicating that Dppa3 might be the genetic factor responsible for the Vc-mediated maintenance of proper imprinting at the Dlkl-Dio3 cluster.

Collectively, our data show that Dppa3 is a genetic component essential for establishing 
exclusively $G t / 2^{\text {on }}$ iPSCs, which show full pluripotential characteristics. Based on these results, we propose that Dppa3 protects the Dlk1-Dio3 imprinting during somatic cells reprogramming. The early expression of Dppa3 either by exogenous expression or by addition of $\mathrm{Vc}$ leads to the binding of Dppa3 to the Dlk1-Dio3 IG-DMR and probably also to other known or unknown genomic regions. Upon binding, Dppa3 counteracts the recruitment of Dnmt3a to this region thereby maintaining the expression of imprinted genes from the Dlk1-Dio3 cluster.

\section{Acknowledgments}

The authors would like to thank Prof. Masatsugu Ohora for providing pMXs-Blimp1 construct. This work was partly supported by the German Research Foundation (Deutsche Forschungsgemeinschaft) DFG SPP_1356 (EN 84/22-1 and ZE 442/4-2), DFG FOR 1041 (EN 84/23-1 and ZE 442/5-2).

\section{References}

1. Grabel, L. Prospects for pluripotent stem cell therapies: into the clinic and back to the bench. $J$ Cell Biochem 113, 381-7 (2012).

2. Takahashi, K. \& Yamanaka, S. Induction of pluripotent stem cells from mouse embryonic and adult fibroblast cultures by defined factors. Cell 126, 663-76 (2006).

3. Takahashi, K. et al. Induction of pluripotent stem cells from adult human fibroblasts by defined factors. Cell 131, 861-72 (2007).

4. Chin, M.H. et al. Induced pluripotent stem cells and embryonic stem cells are distinguished by gene expression signatures. Cell Stem Cell 5, 111-23 (2009).

5. Doi, A. et al. Differential methylation of tissueand cancer-specific $\mathrm{CpG}$ island shores distinguishes human induced pluripotent stem cells, embryonic stem cells and fibroblasts. Nat Genet 41, 1350-3 (2009).

6. Hussein, S.M. et al. Copy number variation and selection during reprogramming to pluripotency. Nature 471, 58-62 (2011).

7. Stadtfeld, M. et al. Aberrant silencing of imprinted genes on chromosome $12 \mathrm{qF} 1$ in mouse induced pluripotent stem cells. Nature 465, 175-81 (2010).

8. Liu, L. et al. Activation of the imprinted Dlk1-Dio3 region correlates with pluripotency levels of mouse stem cells. $J$ Biol Chem 285, 19483-90 (2010).

9. Stadtfeld, M. et al. Ascorbic acid prevents loss of Dlk1-Dio3 imprinting and facilitates generation of all-iPS cell mice from terminally differentiated B cells. Nat Genet 44, 398-405, S1-2 (2012).

10. Spahn, L. \& Barlow, D.P. An ICE pattern crystallizes. Nat Genet 35, 11-2 (2003).

11. Ferguson-Smith, A.C. \& Surani, M.A. Imprinting and the epigenetic asymmetry between parental genomes. Science 293, 1086-9 (2001).

12. Tycko, B. \& Efstratiadis, A. Genomic imprinting: piece of cake. Nature 417, 913-4 (2002).

13. Brandeis, $M$. et al. The ontogeny of allele-specific methylation associated with imprinted genes in the mouse. EMBO J 12, 3669-77 (1993).

14. Allegrucci, C., Thurston, A., Lucas, E. \& Young, L. Epigenetics and the germline. Reproduction 129, 137-49 (2005).

15. Lucifero, D., Mertineit, C., Clarke, H.J., Bestor, T.H. \& Trasler, J.M. Methylation dynamics of imprinted genes in mouse germ cells. Genomics 79, 530-8 (2002).

16. Arnaud, P. Genomic imprinting in germ cells: imprints are under control. Reproduction 140, 411-23 (2010).

17. Bourc'his, D., Xu, G.L., Lin, C.S., Bollman, B. \& Bestor, T.H. Dnmt3L and the establishment of 
maternal genomic imprints. Science 294, 2536-9 (2001).

18. Hata, K., Okano, M., Lei, H. \& Li, E. Dnmt3L cooperates with the Dnmt3 family of de novo DNA methyltransferases to establish maternal imprints in mice. Development 129, 1983-93 (2002).

19. Kaneda, M. et al. Essential role for de novo DNA methyltransferase Dnmt3a in paternal and maternal imprinting. Nature 429, 900-3 (2004).

20. Jelinic, P., Stehle, J.C. \& Shaw, P. The testis-specific factor CTCFL cooperates with the protein methyltransferase PRMT7 in H19 imprinting control region methylation. PLOS Biol 4, e355 (2006).

21. Kou, Y.C. et al. A recurrent intragenic genomic duplication, other novel mutations in NLRP7 and imprinting defects in recurrent biparental hydatidiform moles. Mol Hum Reprod 14, 33-40 (2008).

22. Li, X. et al. A maternal-zygotic effect gene, Zfp57, maintains both maternal and paternal imprints. Dev Cell 15, 547-57 (2008).

23. Meyer, E. et al. Germline mutation in NLRP2 (NALP2) in a familial imprinting disorder (Beckwith-Wiedemann Syndrome). PLoS Genet 5, e1000423 (2009).

24. Murdoch, S. et al. Mutations in NALP7 cause recurrent hydatidiform moles and reproductive wastage in humans. Nat Genet 38, 300-2 (2006).

25. Nakamura, T. et al. PGC7/Stella protects against DNA demethylation in early embryogenesis. Nat Cell Biol 9, 64-71 (2007).

26. Xu, X. et al. Stage-specific germ-cell marker genes are expressed in all mouse pluripotent cell types and emerge early during induced pluripotency. PLoS One 6, e22413 (2011).

27. Nishikawa, K. et al. Blimp1-mediated repression of negative regulators is required for osteoclast differentiation. Proc Natl Acad Sci U S A 107, 3117-22 (2010).
28. Okita, K., Ichisaka, T. \& Yamanaka, S. Generation of germline-competent induced pluripotent stem cells. Nature 448, 313-7 (2007).

29. Khromov, T. et al. Global and gene-specific histone modification profiles of mouse multipotent adult germline stem cells. Mol Hum Reprod 17, 166-74 (2011).

30. Zechner, U. et al. Comparative methylation profiles and telomerase biology of mouse multipotent adult germline stem cells and embryonic stem cells. Mol Hum Reprod 15, 345-53 (2009).

31. Dressel, R. et al. Multipotent adult germ-line stem cells, like other pluripotent stem cells, can be killed by cytotoxic $\mathrm{T}$ lymphocytes despite low expression of major histocompatibility complex class I molecules. Biol Direct 4, 31 (2009).

32. Dressel, R. et al. Pluripotent stem cells are highly susceptible targets for syngeneic, allogeneic, and xenogeneic natural killer cells. Faseb J 24, 2164-77 (2010).

33. Nakagawa, M. et al. Generation of induced pluripotent stem cells without Myc from mouse and human fibroblasts. Nat Biotechnol 26, 101-6 (2008).

34. Smorag, L. et al. MicroRNA signature in various cell types of mouse spermatogenesis: Evidence for stage-specifically expressed miRNA-221, -203 , and $-34 b-5 p$ mediated spermatogenesis regulation. Biol Cell (2012).

35. Wang, T. et al. The histone demethylases Jhdm la/1b enhance somatic cell reprogramming in a vitamin-C-dependent manner. Cell Stem Cell 9, 575-87 (2011).

36. Payer, B. et al. Stella is a maternal effect gene required for normal early development in mice. Curr Biol 13, 2110-7 (2003).

37. Sato, M. et al. Identification of PGC7, a new gene expressed specifically in preimplantation embryos and germ cells. Mech Dev 113, 91-4 (2002). 
38. Nakamura, T. et al. PGC7 binds histone $\mathrm{H} 3 \mathrm{~K} 9 \mathrm{me} 2$ to protect against conversion of $5 \mathrm{mC}$ to $5 \mathrm{hmC}$ in early embryos. Nature $\mathbf{4 8 6}, 415-9$ (2012).
39. Esteban, M.A. et al. Vitamin C enhances the generation of mouse and human induced pluripotent stem cells. Cell Stem Cell 6, 71-9 (2010). 


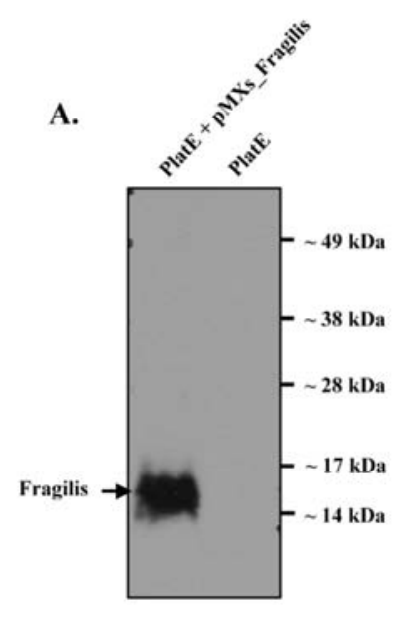

Figure S1. Verification of pMXs-Fragilis construct. Western blot showing the expression of Fragilis in pMXs-Fragilis construct-transfected PlatE cells, whereas PlatE cells alone (control) served as a control.

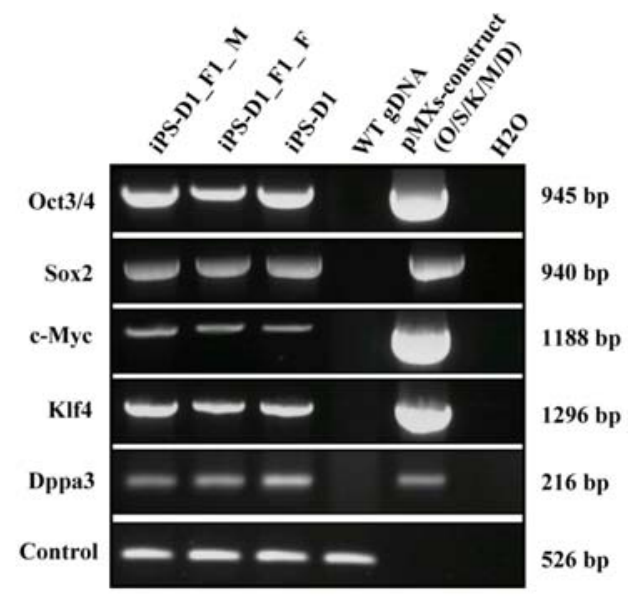

Figure S2. Confirmation of iPS-D germline transmission. Genotyping PCR showing the presence of OSKM+D constructs in two offspring (iPS-D1_F1_M and iPS-D1_F1_F) derived from the iPS-D1 chimera. Genomic DNA from iPS-D1 and wild-type ESCs (WT gDNA) served as positive and negative controls, respectively. Similarly, plasmid DNAs of OSKMD constructs (pMXs-construct) and water served as positive and negative controls, respectively. The sizes of the PCR fragments are indicated on the right side. 


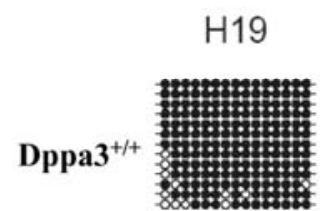

(94\%)

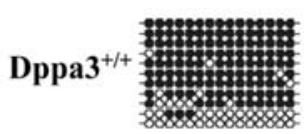

$(77 \%)$

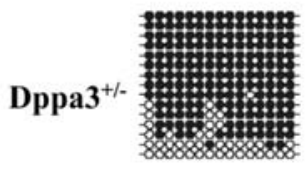

$(82 \%)$

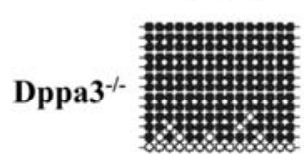

(89\%)

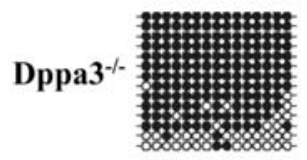

$(82 \%)$
Dlk1/Gtl2

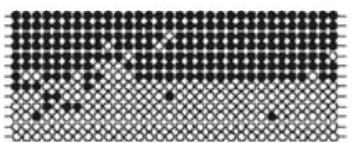

(53\%)

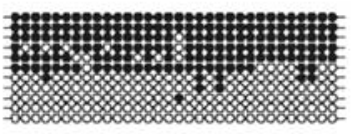

$(52 \%)$

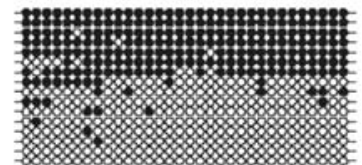

$(46 \%)$

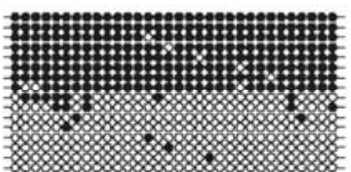

$(52 \%)$

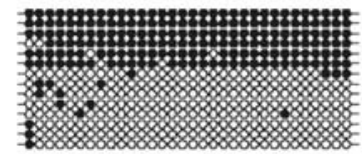

$(45 \%)$

$$
\text { Rasgrf1 }
$$

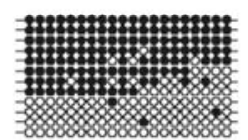

$(57 \%)$

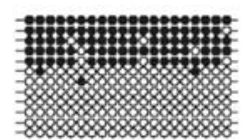

$(40 \%)$

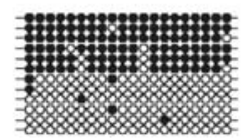

$(49 \%)$

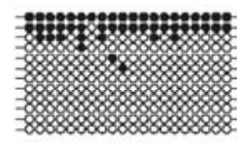

$(20 \%)$

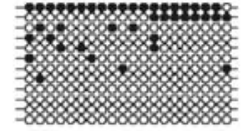

$(18 \%)$

Figure S3. Analysis of the Dlk1-Dio3 imprinting status in Dppa3-deficient ESCs. DNA methylation analysis for the paternally imprinted H19,Dlkl-Dio3, and Rasgrfl loci in wild-type $\left(D p p a 3^{+/+}\right)$, heterozygous $\left(D p p a 3^{+/}\right)$, and homozygous $\left(\right.$ Dppa3 $\left.^{-/}\right)$ESCs. 


\subsection{Mouse Dazl and its novel splice variant function in translational repression of target mRNAs in embryonic stem cells}

Although pluripotent cells are known to express several PrM marker genes, their functional relevance to pluripotency is largely unknown. To unveil the function of PrM marker genes in pluripotent cells, in the third part of this thesis, we choose Dazl, a PrM marker gene, as a candidate and elucidated its function in ESCs. During the course of this study we identified a novel Dazl splice variant (Dazl_48) in mouse ESCs and subsequently found it to be expressed in various pluripotent cell types as well as at different stages of testicular development. Protein sub-cellular localization studies revealed a diffused cytoplasmic and large granular localization pattern for Dazl_ $\Delta 8$, which is similar to the pattern observed for Dazl full length (Dazl_FL) protein. Moreover, the studies using alteration of Dazl expression in ESCs indicated a role for Dazl in the negative translation regulation of both known and unknown target genes. The translation repressive role of Dazl in ESCs but not in germ line derived GC1 cells was further confirmed in luciferase reporter assays. Furthermore, we identified several specific as well as common targets for both Dazl isoforms in ESCs through RNA-binding immunoprecipitation followed by whole genome transcriptome analysis. Collectively, our results highlight that Dazl might function as a translational repressor at physiological level to maintain the balance between germ cell lineage commitment and pluripotency of ESCs.

Authors: Xingbo Xu, Xiaoying Tan, Qiong Lin, Bernhard Schmidt, Wolfgang Engel and D.V. Krishna Pantakani

Status: Submitted to BBA-Gene Regulatory Mechanisms journal.

\section{Author contributions to the work:}

1. Xingbo $\mathrm{Xu}$ : Conception and design of experiments, data collection, data analysis, data interpretation, helped in drafting the manuscript.

2. Xiaoying Tan: Data collection, data analysis.

3. Qiong Lin: Microarray data analysis.

4. Bernhard Schmidt: Design of experiments, data analysis.

4. Wolfgang Engel: Conception and design of experiments, financial support, helped in drafting the manuscript.

5. D.V. Krishna Pantakani: Conception and design of experiments, data interpretation, drafted the manuscript. 


\title{
Mouse Dazl and its novel splice variant functions in translational
} repression of target mRNAs in embryonic stem cells

\author{
Xingbo Xu', Xiaoying Tan ${ }^{1}$, Qiong Lin ${ }^{2}$, Bernhard Schmidt ${ }^{3}$, \\ Wolfgang Engel ${ }^{1}$ and D.V. Krishna Pantakani ${ }^{1 . \$}$ \\ 'Institute of Human Genetics, University of Goettingen, Goettingen, Germany. ${ }^{2}$ Institute for Biomedical Engineering, RWTH Aachen \\ University Medical School, Aachen, Germany. ${ }^{3}$ Department of Biochemistry II, University of Goettingen, Goettingen, Germany. \\ \$Correspondence: D. V. Krishna Pantakani, Tel: +49-551-399302; Fax: +49-551-399303; E-mail: krishna.if1@gmail.com
}

\begin{abstract}
Dazl (deleted in azoospermia-like) is an RNA binding protein that is important for germ cell differentiation in vertebrates. In the present study, we report the identification of a novel Dazl isoform (Dazl_48) that results from alternative splicing of exon 8 of mouse Dazl. We observed the expression of Dazl_ $\Delta 8$ in various pluripotent cell types, but not in somatic cells. Furthermore, the Dazl_ 48 splice variant was expressed along with the full-length isoform of Dazl (Dazl_FL) throughout male germ-cell development and in the ovary. Sub-cellular localization studies of Dazl_ $\Delta 8$ revealed a diffused cytoplasmic and large granular pattern, which is similar to the localization patterns of Dazl_FL protein. In contrast to the well documented translation stimulation function in germ cells, overexpression and downregulation studies of Dazl isoforms (Dazl_FL and Dazl_48) revealed a role for Dazl in the negative translational regulation of $M v h$, a known target of Dazl, as well as Oct3/4 and Sox2 in embryonic stem cells (ESCs). In line with these observations, a luciferase reporter assay with the 3'UTR's of Oct3/4 and Mvh confirmed the translational repressive role of Dazl isoforms in ESCs but not in germ cells derived cell line GC-1. Further, we identified several putative target mRNAs of Dazl_FL and Dazl_ $\Delta 8$ in ESCs through RNA-binding immunoprecipitation followed by whole genome transcriptome analysis. Collectively, our results show a translation repression function of Dazl in pluripotent stem cells.
\end{abstract}

Keywords: ESCs, Dazl, splice variant, translation repression, RNA binding

Abbreviations: maGSCs-multipotent adult germline stem cells; EGCs-embryonic germ cells; iPSCs-induced pluripotent stem cells; ECCs- embryonic carcinoma cells; GC-1- a spermatogonia cell line; RIP- RNA-binding protein immunoprecipitation

\section{Introduction}

The DAZ (Deleted in Azoospermia) family of proteins, DAZ, DAZL (DAZ-Like), and BOULE play a crucial role in the gametogenesis of metazoans by regulating the translation of target messenger RNAs (mRNAs). These proteins are known to contain a RNA recognition motif (RRM) that mediates the interaction with target mRNAs, as well as characteristic single or multiple repeats of the
DAZ motif and a proline rich (PR) region. BOULE is evolutionarily conserved and found in all metazoans; whereas, DAZL is only present in vertebrates $[1,2]$. Interestingly, DAZ originated from a duplication transposition of the DAZL gene to the distal long arm of the Y chromosome in multiple copies and is found only in Old World monkeys and humans [3, 4]. In humans, the deletion of the DAZ cluster is believed to cause azoospermia and oligospermia in $5-10 \%$ of infertile men $[3,5]$. Loss of function 
studies of autosomal members of Daz lead to a failure to produce mature gametes in several model organisms [6-9].

The mouse Dazl codes for a germ cell-specific protein present in both the nucleus and cytoplasm of fetal gonocytes [10]. However, in spermatogonial stem cells (SSCs), Dazl is localized mainly to the nucleus and relocates into the cytoplasm during meiosis [10]. Concomitant with the specific expression in germ cells, Dazl has been shown to be important for germ cell development in diverse species, including humans $[6,7,9,11-14]$. The deletion of mouse Dazl (mDazl) results in loss of germ cells in both male and female gonads, where male germ cells are lost partly due to increased apoptosis, reduced expression of germ cell markers, and aberrant chromatin structure $[9,11,15]$. In addition, it has been reported that Dazl deficiency leads to the reduction of post-migratory PGCs (primordial germ cells), and a failure to erase and re-establish genomic imprints, as well as defects in meiotic progression in mice[16].

Apart from germ cells, several reports have documented the cytoplasmic expression of Dazl in pluripotent embryonic stem cells (ESCs) [17-19]. Furthermore, Dazl depleted PGCc show aberrant expression of pluripotency-related genes and failed to establish pluripotent embryonic germ cells (EGCs), thus highlighting the role of Dazl in the establishment of pluripotency [16]. Consistent with the role of Dazl in germ cells development, Yu et al., (2009) have shown that the forced expression of Dazl leads to the differentiation of mouse ESCs into cells resembling sperm cells and oocytes, in vitro [20]. Strikingly, these in-vitro derived sperm-like cells can fertilize wild-type oocytes, which further underwent embryonic development until the 8-cells stage [20]. Collectively, these results reinforce the idea that Dazl might function as a master regulator for germ cell specification and differentiation.

The ability of Dazl to control germ cell development is attributed to its potential role in the translational regulation of known and unknown target mRNAs [21-26]. Molecular analysis of the human and mouse genomes over the last decade revealed alternative splicing as one of the most essential and common mechanisms of generating a large repertoire of mRNA and protein isoforms, which is occurring in $>80 \%$ of genes $[27,28]$. The protein products of these alternative isoforms have changed structures and functions, leading to a more complex proteome. Unfortunately, nothing is known about the Dazl splice variants and their possible functions in translation regulation. In the present study, we report the alternative splicing of exon 8 of mDazl that leads to the generation of the novel splice variant, Dazl_ $\Delta 8$. This novel transcript was detectable in all analyzed pluripotent cell types, adult mouse testis, as well as in ovary, albeit at low levels compared to Dazl_FL expression. Furthermore, we observed the expression of Dazl_ $\Delta 8$ throughout mouse testicular development, specifically in germ cells, not in testicular somatic cells. Interestingly, overexpression and downregulation studies of Dazl isoforms (Dazl_FL and Dazl_ $\Delta 8$ ), and luciferase reporter assays using the 3'UTRs of Oct $3 / 4$ and Mvh revealed a negative translational role for Dazl in ESCs. In addition, we performed RNA-binding protein immunoprecipitation (RIP) assays on ESCs transiently overexpressing E2-epitope tagged Dazl_ $\Delta 8$ or Dazl_FL and could identify several specific as well as common mRNA targets of Dazl isoforms.

\section{Materials and methods}

\section{Animals and cell culture}

The mouse mutants $W / W^{V}$ [29], Tfm/y [30], olt/olt [31] and $q k / q k$ [32] were purchased from the Jackson Laboratory, whereas $l e y I-l^{-*}$ was generated in-house [33]. All animal experiments were performed according to protocols approved by the Institutional Ethical Committee of University of Goettingen. Derivation and cultivation of mouse ESC, 
maGSC, EGC, iPSC, ECC and GC-1 cell lines have been described previously [19, 34]. For transient overexpression experiments, ESCs were seeded in KO-DMEM supplemented with KO-serum replacement (Life technologies) at a density of $2 \times$ $10^{5}$ cells/well of a 6-well plate containing feeder layer. Five hours after plating, the cells were transfected with indicated constructs using Lipofectamine-2000 (Life technologies), according to manufacturer's instructions. After $3 \mathrm{~h}$ of transfection, the medium was changed back to standard ESCs culture medium and the cells were harvested after $48 \mathrm{~h}$ of culture. Dazl downregulation studies were performed as described [19]. Briefly, ESCs were seeded at a density of $2 \times 10^{5}$ cells/ well of a 6-well plate containing feeder. After $5 \mathrm{~h}$ of plating, the cells were transfected with either of two independent Dazl siRNA, Dazl_siRNA_exon7 (NM_010021.4_stealth_726, Life technologies) and Dazl_siRNA_exon11 (NM_010021.4_stealth_1056, Life technologies), or scrambled siRNA (Stealth_control, Life technologies) using Lipofectamine-2000 (Life technologies). After $3 \mathrm{~h}$ of transfection, the medium was changed to standard ESCs culture medium and allowed to grow for $24 \mathrm{~h}$. The next day, transfection was repeated and cells were harvested after an additional $24 \mathrm{~h}$ of culture. The sequences of Stealth siRNA duplex oligoribonucleotides are listed in Table S2.

\section{Construction of the Dazl overexpression and luciferase vectors}

The mouse Dazl open reading frame was amplified by Takara LA Taq Polymerase (Clontech) using mESC cDNA as the template. The PCR products were cloned into pGEM-T Easy vector (Promega). After sequencing verification, fragments were sub-cloned into the phEF1 a expression vector to generate phEF1 a -Dazl_FL (lacking an epitope tag,

Dazl_F: GGATCCGATGTCTGCCACAACTTCTGAG and Dazl_R:

\section{GCGGCCGCTTAGCAGAGATGATCAGATTTAA}

G). In addition, PCR and subsequent cloning was performed using primers containing C-terminal E2-epitope tag (Dazl-E2_F: GGATCCGATGTCTGCCACAACTTCTGAG and Dazl-E2_R:

GCGGCCGCTTAGCGATCTCTAAAATCAGAAG

AAGTACTTGAACCACCGCAGAGATGATCAGA

TTTAAG) to generate phEF1 a -Dazl_FL-E2 and phEF1 a -Dazl_A8-E2.

To generate Luciferase reporter constructs for Oct3/4 and Mvh 3' UTRs, PCR and subsequent cloning was performed using cDNA $(1 \mu \mathrm{g})$ from ESCs as a template to amplify Oct3/4 and Mvh $3^{\prime}$ UTR sequences. Primers used for PCR amplification of $3^{\prime}$ UTRs are provided in the supplementary Table S3. The amplified PCR product was gel-purified and subcloned into pGEM-T Easy vector (Promega). The inserted sequences were first sequence verified and then excised with SacI and HindIII restriction enzymes (Life technologies), gel-purified, and cloned into the pMIR-REPORT miRNA luciferase reporter vector (Ambion), referred to as pMIR-Oct3/4-3'UTR and pMIR-Mvh-3'UTR.

\section{Protein extraction and Western blotting}

Proteins were extracted from cells and tissues using lysis buffer (10mM Tris- $\mathrm{HCl}(\mathrm{pH} 8.0), 1 \mathrm{mM}$ EDTA, $2.5 \%$ SDS, $100 \mathrm{mM}$ PMSF) containing protease inhibitor cocktail (Roche). Protein samples were resolved on 4-12\% SDS-PAGE and transferred onto nitrocellulose membrane (Amersham Biosciences). Membranes were processed using standard Western blot protocols, and signals were detected using a chemiluminescent kit (Santa Cruz Biotechnology). Antibody sources are listed in supplementary Table S4.

\section{RNA extraction, RT-PCR and qPCR}

Total RNA was extracted from cells and mouse testis samples using Trizol Reagent (Life technologies) following the manufacturers' protocols. 
Total RNA was digested with DNaseI (Sigma) and used for cDNA synthesis using the SuperScriptII system (Life technologies). For qPCR analysis, diluted cDNA (1/10) was used as a template in QuantiFast SYBR Green (Qiagen) reaction and run in ABI 7900HT Real-Time PCR System (Applied Biosystems). Primers used in RT-PCR and qPCR are listed in supplementary Tables S5 and S6, respectively.

\section{Immunofluorescence staining}

ESCs, NIH-3T3, and GC-1 cells grown on round cover slips were transfected with indicated constructs using Lipofectamine 2000 (Life technologies). After $24 \mathrm{~h}$ of transfection, the cells were processed for immunofluorescence staining, as described [35]. Microscopic images were acquired using Olympus BX60 fluorescent microscope equipped with UplanFI 20X/0.50 objective lens and Cell $^{\wedge} \mathrm{F}$ program. The acquired images were processed using the same $\mathrm{Cell}^{\wedge} \mathrm{F}$ software.

\section{Luciferase reporter assay}

Luciferase reporter assay was performed as described previously [36]. Briefly, one day before transfection, ESCs or GC-1 were trypsinized and transferred to a fresh well of a 6 well plate at a density of $1 \times 10^{5}$ cells/well. Next day, the cells were transiently transfected in duplicates with indicated luciferase vector (pMIR-Oct3/4-3'UTR or pMIR-Mvh-3'UTR), pMIR-Report $\beta$-Gal (control plasmid for use in normalization of the results) (Life technologies) and with either phEF1 $\alpha$-Dazl_FL-E2 or phEF1 $\alpha$-Dazl_ $\Delta 8$-E2 using Lipofectamine 2000 (Life technologies). The optimized DNA transfection amount per each vector was $2.5 \mu \mathrm{g}$. After $48 \mathrm{~h}$ of transfection, the cells were lysed and assayed for both luciferase and $\beta$-Gal activity using Luciferase Assay System (Promega) and Galacto-Light Plus (Applied Biosystems), respectively. Normalization was performed with luciferase/ $\beta$-gal activity in cells transfected with negative control plasmid.

\section{RIP, microarray analysis and data manipulation}

RNA-binding protein immunoprecipitation (RIP) assays were performed using EZ-Magna RIP Kit (Millipore) according to manufacturer's instructions. Briefly, ESCs ( $\sim 5 \times 10^{6}$ cells) were transfected with E2-tagged isoform specific constructs (Dazl_FL-E2 or Dazl_ $\Delta 8$-E2). After $48 \mathrm{~h}$ of transfection, the cells were washed two times with ice-cold PBS and transferred into a falcon tube by scrapping. After centrifugation, the pellet was re-suspended with an equal volume of RIP lysis buffer and mixed properly until the cells appeared homogeneous. After $5 \mathrm{~min}$ of incubation, the protein-RNA complex was obtained by centrifugation at $11,000 \times \mathrm{g}$ for $10 \mathrm{~min}$ at $4{ }^{\circ} \mathrm{C}$. The protein-RNA complex $(100 \mu \mathrm{l})$ was used for each immunoprecipitation and pulled down by a beads-antibody complex consisting of $6 \mu \mathrm{l}$ of E2-antibody coupled to magnetic beads. After $3 \mathrm{~h}$ of incubation at $4^{\circ} \mathrm{C}$, the protein was digested by using proteinase- $\mathrm{K}$ at $55{ }^{\circ} \mathrm{C}$ for $30 \mathrm{~min}$. Then, RNA purification was carried out using $400 \mu \mathrm{l}$ phenol:chloroform:isoamyl alcohol (125:24:1 pH 4.3) (Sigma-Aldrich) and precipitated with Salt Solution I, II, and precipitate enhancer. The RNA pellet was washed with $80 \%$ ethanol and dissolved in $20 \mu \mathrm{l}$ of RNase-free water and quantified using the NanoDrop ND-1000 (Thermo Scientific). The precipitated RNA (RIPed-RNA) samples were used in cDNA microarray as described previously [37].

The microarray raw data was processed as previously described [38]. Briefly, the raw data was quantile normalized and each gene's intensity value was given a percentile rank based on the ratio of the gene's intensity compared to the intensities for the entire array (the gene with the greatest intensity value has the highest rank). This rank was transformed to a percent relative to the other genes on the array, and each gene given a median percentile rank across each set of RIP experiments. Top ranked genes $(>0.98)$ were selected. As a result, 116 genes were identified as Dazl_ $\Delta 8$ targets and 626 genes as Dazl_FL targets. Out of the 116 Dazl_ $\Delta 8$ and 626 Dazl_FL targets, 85 
genes were identified as common targets to both Dazl isoforms.

\section{Mass spectrometry analysis}

The ESC protein extracts were immunoprecipitated using anti-Dazl antibodies and the resulting protein complex was resolved on $4-12 \%$ SDS -PAGE and stained using coomassie blue staining solution. The indicated protein bands were excised out of the gel, the proteins reduced and carbamidomethylated, in-gel digested with trypsin and analyzed by LC-MALDI mass spectrometry as described [39]. Combined mass lists from MS/MS spectra were used for a database search in the NCBInr Protein database using Mascot 2.4 (Matrix Science).

\section{BNPS-Skatole cleavage of protein extracts}

BNPS-Skatole cleavage experiments were carried out as previously described [40]. Briefly, ESCs grown to a confluence in a well of six-well plate were harvested and the resulting cell pellet was resuspended in $75 \mu \mathrm{l}$ of PBS and subjected to five rounds of freeze-thaw cycles using dry ice/ethanol. The cell insoluble fraction was removed by centrifugation at $20,000 \times \mathrm{g}$ for $5 \mathrm{~min}$ at $4{ }^{\circ} \mathrm{C}$. The soluble protein fraction was denatured with $75 \mu \mathrm{l}$ of $8 \mathrm{M}$ urea (dissolved in PBS). Then, $250 \mu \mathrm{l}$ of freshly prepared BNPS-Skatole $(1.6 \mathrm{mg} / \mathrm{ml})$ in glacial acetic acid was added to the lysates. The reactions were kept overnight at room temperature (RT) in dark. Proteins were precipitated by adding $1.2 \mathrm{ml}$ of cold acetone and incubation at $-20{ }^{\circ} \mathrm{C}$ for $2 \mathrm{~h}$, followed by centrifugation at $20,000 \times \mathrm{g}$ for $10 \mathrm{~min}$ at $4{ }^{\circ} \mathrm{C}$. The supernatant was aspirated and the pellet was completely dried at RT. Then the samples were dissolved in $35 \mu \mathrm{l}$ of $8 \mathrm{M}$ urea in PBS and inactivated at $95{ }^{\circ} \mathrm{C}$ for $10 \mathrm{~min}$. Proteins samples were prepared by adding equal volume of $2 \times$ non-reducing Laemmli buffer and subsequent incubation at $95{ }^{\circ} \mathrm{C}$ for $5 \mathrm{~min}$ and analyzed by SDS-PAGE followed by Western blot.

\section{Statistical Analysis}

All $\mathrm{qPCR}$ data for RNA expression analysis (two or more biological replicates with three technical replicates each) were calculated using the standard curve method and are expressed as mean \pm SD. For statistical significance calculations, 2way ANOVA or Student's T-test (GraphPad Prism 4.0) were used.

\section{Results}

Identification of new mouse Dazl isoform, Dazl_48

The genomic architecture of mouse Dazl and the details of the encoded protein and its functional domains are depicted in Fig. 1A. The mouse Dazl gene spans $\sim 14 \mathrm{~kb}$ and consists of 11 exons, which encodes a 298 aa polypeptide, hereafter referred as Dazl_FL (Fig. 1A). The Dazl protein contains an RNA recognition motif (RRM), a proline rich region $(\mathrm{PR})$ in addition to the characteristic DAZ (deleted in azoospermia) domain (Fig. 1B). In Western blot analysis of protein extracts from testis and pluripotent ESCs, a second band of $\sim 30 \mathrm{kDa}$ (marked with asterisk) was detected along with the known $\sim 32 \mathrm{kDa}$ band of Dazl_FL (Fig. 1C). To analyze whether Dazl exists as an alternatively spliced isoform, we performed RT-PCR on cDNA derived from ESCs using open reading frame (ORF) specific primers and detected a second fragment ( $\sim 850$ bp) along with the expected $\sim 900$ bp band corresponding to the Dazl_FL (Fig. 1 D). We then cloned these two fragments into pGEM-T Easy vector and had them sequenced. The sequencing results revealed an alternatively spliced isoform of Dazl with a deletion of exon8 (51 bp), referred to as Dazl_ 48 (Fig. 1A, E). Analysis of the protein sequence revealed that Dazl_ $\Delta 8$ isoform is specifically missing a 17 aa region in the PR domain (Fig. 1B). To determine whether this isoform is expressed in pluripotent cell types other than ESCs, 
A.

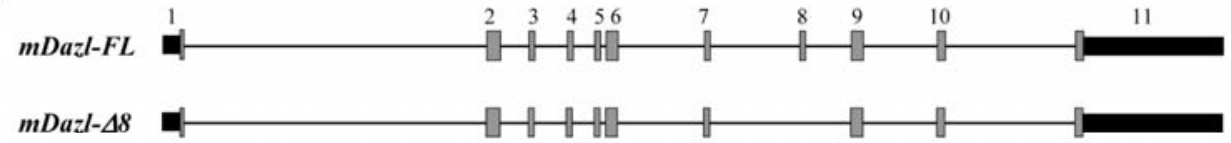

B.

mDazl-FL

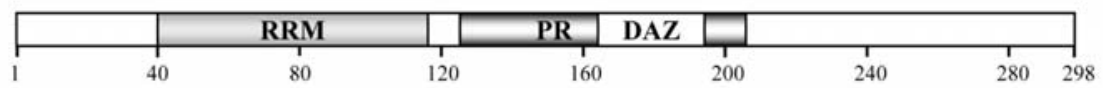

mDazl- $\Delta 8$

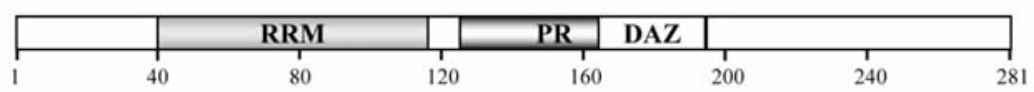

c.

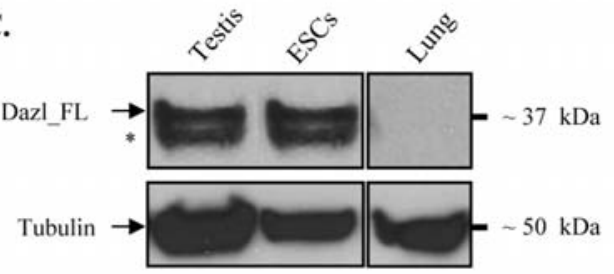
Dazl_FL
557 GTTPATAACTACCAGATGCCACCGCAGTGGCCTGCTGGAGAGCAGAGGAGTTATGTTATACCTCCGGCTTATACAACT 824
Dazl_48

D.

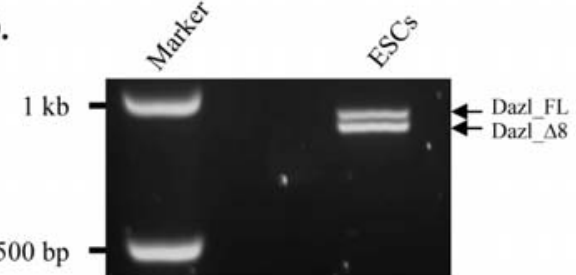

Sequence

F.

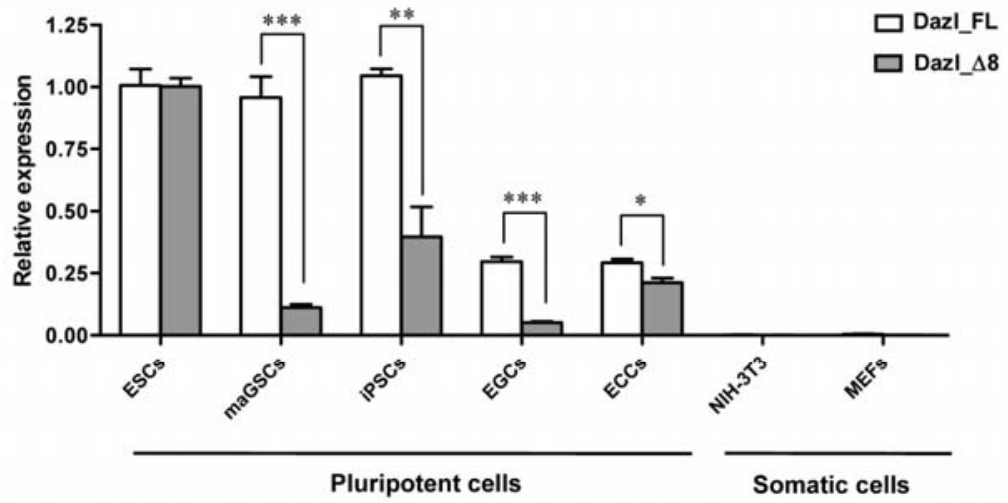

Figure 1. Identification and expression analysis of novel Dazl splice variant. (A) Schematic diagram showing the genomic architecture of mouse $\mathrm{Dazl}$ and its novel splice variant. Numerics on the top indicate the exon number, black rectangles - untranslated regions, grey - coding exons. (B) Protein structural domains of Dazl_FL and Dazl_ 48 . Amino acid positions along the protein are indicated below. RRM - RNA recognition motif, PR - Proline-rich region, and DAZL - DAZ like domain. (C) Western blot for Dazl expression in testis, embryonic stem cell (ESCs), and lung. The Dazl_FL isoform was indicated with arrow. Asterisk denotes the additional band and is most likely the Dazl_ $\Delta 8$. The blot was re-probed with tubulin antibody to verify the 
protein quantities. (D) RT-PCR analysis and subsequent agarose gel electrophoresis showing two PCR products, Dazl_FL, and Dazl_48. (E) Pairwise alignment showing the nucleotide sequences of Dazl splice variants along with the Dazl_FL protein sequence. The nucleotide as well as amino acid numbering is indicated. (F) qRT-PCR showing the expression of Dazl_ 48 in comparison to Dazl_FL in various pluripotent cell types and somatic cells. The error bar represents the mean $\pm \mathrm{SD}$ and the values that are statistically significant $(* \mathrm{p}<0.05$, $* * \mathrm{p}<0.001)$ are indicated.

we analyzed maGSCs, induced pluripotent stem cells (iPSCs), EGCs and embryonal carcinoma cells (ECCs) in comparison with somatic cells, such as NIH-3T3 and MEFs (Fig. 1F). qRT-PCR analysis using primers specific for each Dazl isoform revealed the presence of both Dazl isoforms in all pluripotent cell types, but not in somatic cells (Fig. 1F). The expression level of Dazl_ 48 was similar to that of Dazl_FL in ESCs, while though the Dazl_48 was detectable in all other pluripotent cell types, its levels were significantly lower than the Dazl_FL expression (Fig. 1F). To rule out that the differences in expression levels is due to differences in qPCR primer efficiencies, we verified both Dazl_FL and Dazl_s8-specific primers using standard curve method and found that the PCR amplification efficiencies are similar for both primer sets (Supplementary Fig. S1).

In order to characterize the second band seen in Western blot analysis, we performed immunoprecipitation on ESCs total protein extracts using anti-Dazl antibodies followed by LC-MALDI mass spectrometry analysis. The SDS-PAGE analysis and subsequent coomassie blue staining revealed the precipitation of two putative Dazl protein isoforms along with several other co-precipitated proteins (Supplementary Fig. S2A). When we analyzed these two putative Dazl protein isoforms using mass spectrometry we could identify only the $\mathrm{N}$ - terminal region of Dazl, indicating that the $\mathrm{N}$-terminal region is identical for both Dazl protein isoforms (Supplementary Fig. S2B). To prove that the two Dazl protein isoforms differ in the internal sequence aa 191-207 (present in Dazl_FL and missing in Dazl_ $\Delta 8$ ), we performed a chemical cleavage on total protein extracts of ESCs using 2-(2-nitrophenylsulfenyl)-3-methyl-3-bromoindoleni ne (BNPS-Skatole), which is known to cleave proteins at the $\mathrm{C}$-terminal peptide bond following the tryptophan residue [40]. The amino acid sequence analysis of Dazl contains two tryptophan residues at position 143 and 195, the latter one inside the sequence deleted in Dazl_d8. After cleavage with BNPS-Skatole followed by Western blot analysis using an antibody against the C-terminus of Dazl, two low molecular weight bands differing by about 4 kDa were detected, indicating that Trp195 is missing in the Dazl fragment of the upper band, as predicted for the Dazl_ $\Delta 8$ protein (Supplementary Fig. S2C and D).

\section{Dazl_ $\Delta 8$ isoform is expressed in various testicular developmental stages}

To analyze the expression of Dazl_ 48 during testicular development, we performed qRT-PCR on cDNA samples prepared from various prenatal [15.5dpc (days post coitum), $18.5 \mathrm{dpc}$ ], new born, postnatal [5dpp (days post partum), 10dpp and $15 \mathrm{dpp}]$ and adult testis samples of wild-type animals (Fig. 2A). The qRT-PCR analysis on adult testis samples revealed the presence of the Dazl_ 48 isoform, albeit at $\sim 50 \%$ lower levels compared to Dazl_FL expression levels (Fig. 2A). The Dazl isoform expression analysis in prenatal and new born testis revealed very low expression of both isoforms, in accordance with the absence of meiosis at these stages of testicular development (Fig. 2A). Interestingly, the expression levels of the Dazl_ $\Delta 8$ isoform increased drastically along with Dazl_FL expression in postnatal testis developmental stages 


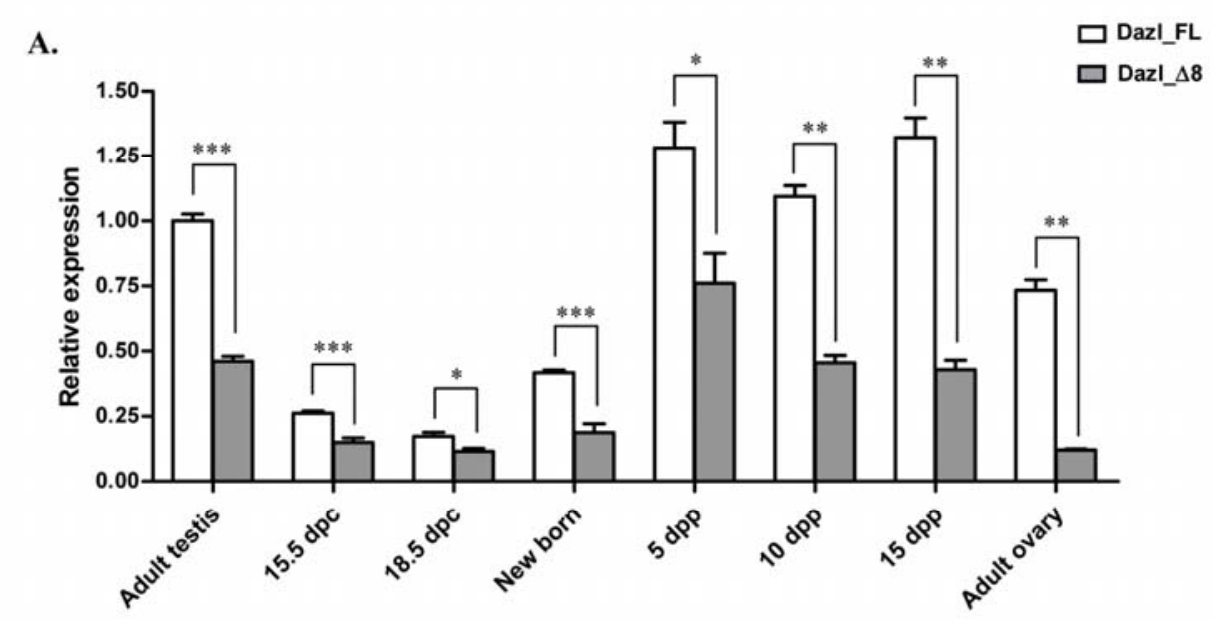

B.

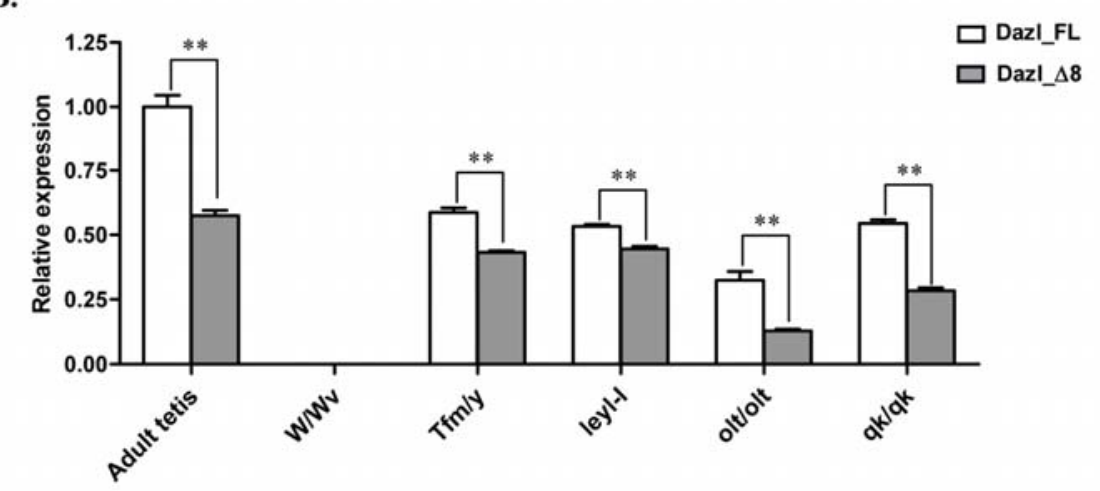

Figure 2. Expression analysis of Dazl isoforms in mouse testis and ovary. (A) qRT-PCR showing the expression of Dazl_FL and Dazl_ 48 in neonatal testis (15.5 dpc and $18.5 \mathrm{dpc}$ ), postnatal testis (newborn, $5 \mathrm{dpp}$, $10 \mathrm{dpp}, 15 \mathrm{dpp}$ ), adult testis, and ovary. dpc- days post coitum, dpp - days post partum. (B) Expression analysis of Dazl_48 and Dazl_FL in spermatogenesis defective adult mouse mutants. White bars - Dazl_FL and grey bars - Dazl_48, expression in both (A) and (B). The error bar represents the mean $\pm \mathrm{SD}$ and the values that are statistically significant $\left(* \mathrm{p}<0.05,{ }^{* *} \mathrm{p}<0.001\right)$ are indicated.

(5-, 10-, 15-dpp), which coincided well with the initiation of meiosis at these stages (Fig. 2A). Again, Dazl_48 expression was significantly lower than Dazl_FL expression at all testicular stages (Fig. 2A). We also analyzed the expression of the Dazl_48 isoform in adult ovary and detected very low levels of expression compared to Dazl_FL (Fig. 2A). To characterize cell-type specific expression, we analyzed cDNA samples from various well characterized spermatogenesis adult mutants, in which spermatogenesis is disrupted at different stages, such as $W / W^{V}$ (which lack all germ cells), $T f m / y$ \& $l e y I-l^{-*}$ (in which spermatogenesis is arrested at the primary spermatocyte stage), and, olt/olt \& $q k / q k$ (in which spermatogenesis is blocked at the spermatid stage) mouse mutant models. qRT-PCR analysis of mutant testes samples indicated that both $\mathrm{Dazl}$ isoforms are undetectable in $W / W^{V}$ mutant testis, but are expressed in all of the other analyzed testis mutants (Fig. 2B). These 
A.

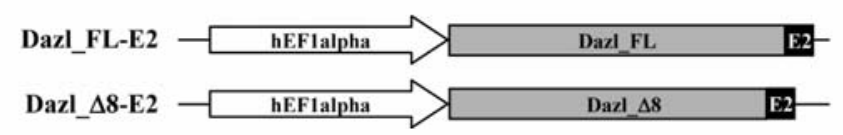

B.
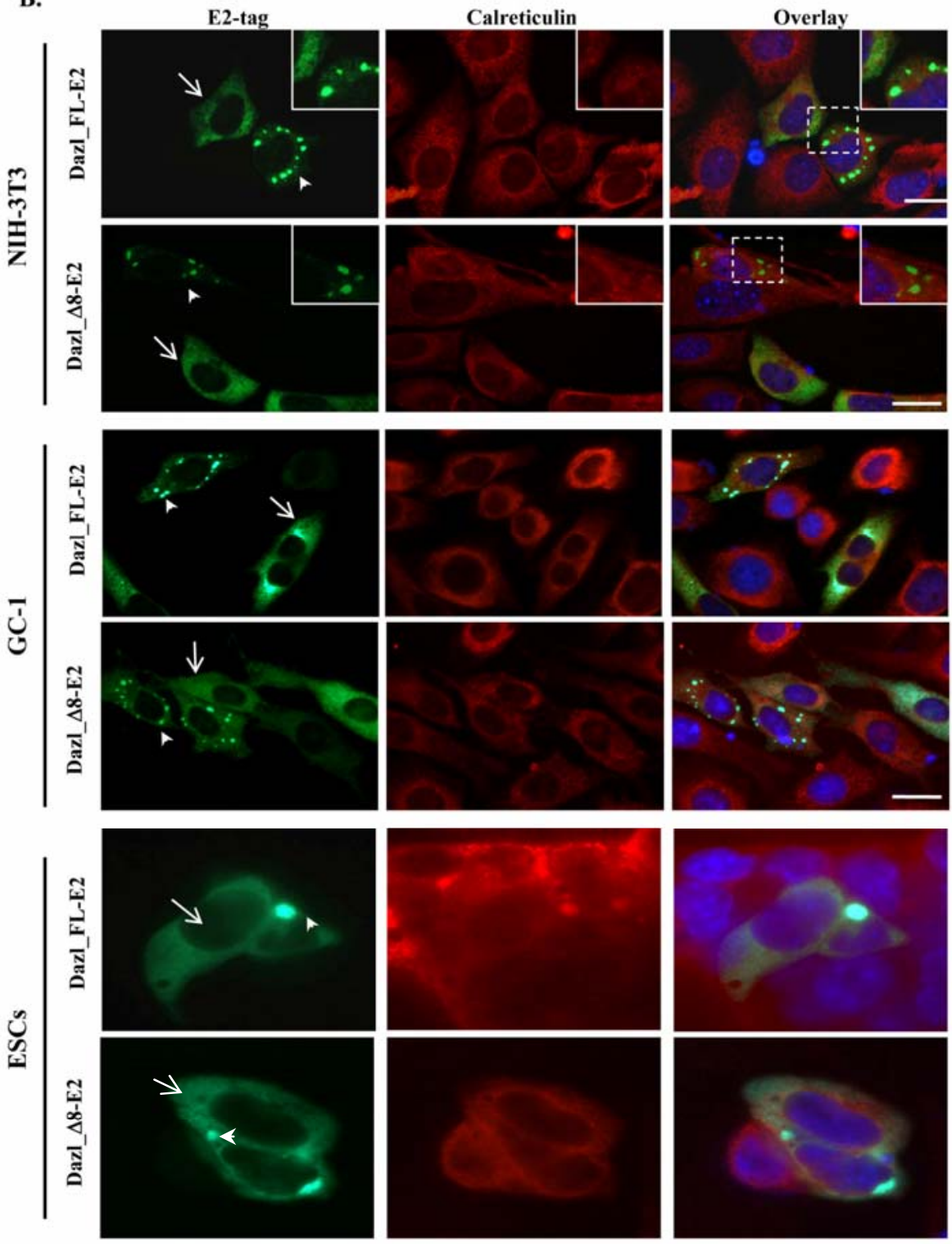

Figure 3. Sub-cellular localization of Dazl isoforms in NIH-3T3 cells. (A) Schematic representation of C-terminally tagged Dazl isoforms. (B) IFC images showing the localization of Dazl_FL-E2 and Dazl_ $\Delta 8$-E2 protein isoforms (green, E2-tag) in NIH-3T3, GC-1 and ES cells. Cells were co-stained with calreticulin (red), an endoplasmic reticulum (ER) marker to visualize the cell morphology. Cells showing diffused cytoplasmic localization are indicated with an arrow, while cells showing large granular localization are highlighted with an arrow head. Insets in NIH-3T3 panels showing the enlarged image of a region indicated with checked line in overlay. 
results suggest the specific expression of Dazl isoforms in either germ stem cells or in different spermatogenic cells or both, but not in somatic cells of testis, such as Sertoli and Leydig cells (Fig. 2B). As was observed in adult testis, the expression levels of Dazl_ $\Delta 8$ isoform were significantly lower than the level of Dazl_FL expression in each of the various mutants (Fig. 2B).

\section{Dazl_ $\Delta 8$ protein localized to the cytoplasm and granular structures, like Dazl_FL protein}

To analyze the sub-cellular localization of Dazl_s8 protein relative to Dazl_FL, we generated mammalian expression constructs with a C-terminal E2-epitope tag (Dazl_FL-E2 and Dazl_d8-E2) (Fig. 3A). Expression of Dazl_FL-E2 in NIH-3T3 cells revealed the localization of Dazl_FL throughout the cytoplasm in $\sim 75 \%$ of transfected cells, while $\sim 20 \%$ of the cells showed large granular localization most probably to the stress granules (SGs) (Fig. 3B). These results are in consistence with previous studies describing the localization of Dazl as being both diffused through the cytoplasm and also in a granular pattern [41] Interestingly, the expression of Dazl_A8-E2 revealed a diffused cytoplasmic localization in $\sim 84 \%$ of cells, while $\sim 12 \%$ of cells showed granular expression pattern (Fig. 3B). We co-stained the cells with calreticulin, an endoplasmic reticulum (ER) marker, to visualize the cellular morphology (Fig. 3B). To rule out any cell type specific localization patterns, we performed Dazl isoforms localization studies in ESCs and germ cells derived GC-1 cell line. Similar to the results observed in NIH-3T3 cells, both ESCs and GC-1 cells revealed both cytoplasmic and granular expression pattern for both Dazl isoforms (Fig. 3B).

\section{Dazl functions in the translational repression of target mRNAs in ESCs}

Since Dazl is a known RNA binding protein and can regulate the translation of specific target mRNAs, we overexpressed Dazl_FL and Dazl_ $\Delta 8$
(Dazl_A8-E2) in ESCs and measured the expression levels of target gene $M v h$ as well as pluripotency-related genes at the RNA and protein levels (Fig. 4A-C). Unexpectedly, overexpression of Dazl_FL resulted in reduced protein expression levels of target gene Mvh as well as Sox2 and Oct $3 / 4$ compared to control cells, while the protein levels of Klf4 was not affected (Fig. 4A). Interestingly, overexpression of Dazl_ $\Delta 8$-E2 also leads to reduced protein levels of Sox 2 , Oct $3 / 4$, and Mvh, but not Klf4 (Fig. 4A), indicating that both Dazl isoforms might function in the translational repression of known as well as unknown target mRNA in ESCs, as opposed to the translation stimulation role they have in germ cells. To rule out that the reduction in protein levels of Mvh, Sox 2 and Oct $3 / 4$ was not due to the transcriptional silencing of these genes, we checked the transcript levels of these genes and found no significant differences between the control cells and those overexpressing the Dazl isoforms (Supplementary Fig. S3A). We also performed downregulation studies for Dazl in ESCs using two independent Dazl siRNA oligos (Dazl_siRNA_exon7 and Dazl_siRNA_exon11), which target the Dazl transcript in exon7 and exon11, respectively. Since exon7 and exon11 are present in both Dazl_FL and Dazl_48 isoforms (Fig. 1A), we expected the simultaneous downregulation of both Dazl protein isoforms. The treatment of ESCs with Dazl_siRNA resulted in a dramatic decrease (up to $80 \%$ ) in the protein levels of both Dazl isoforms, as expected (Fig. 4B). Cells treated with the control_siRNA (scrambled siRNA) did not have a reduction in Dazl protein levels (Fig. 4B). Consistently, the protein expression levels of Sox2, Oct3/4 and Mvh were dramatically increased in Dazl_siRNA treated cells, but not in control_siRNA treated cells (Fig. 4B). The protein expression of Klf4 did not differ between control_siRNA and Dazl_siRNA treated cells, as expected (Fig. 4B). We then analyzed the transcript levels of Sox 2 , Oct $3 / 4$ and Mvh in 
A.

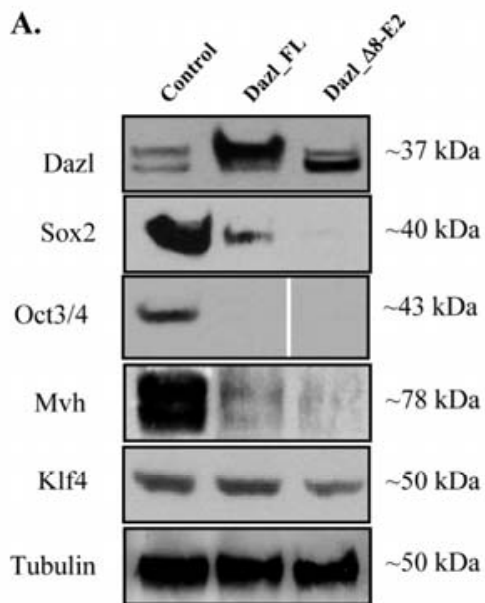

C.

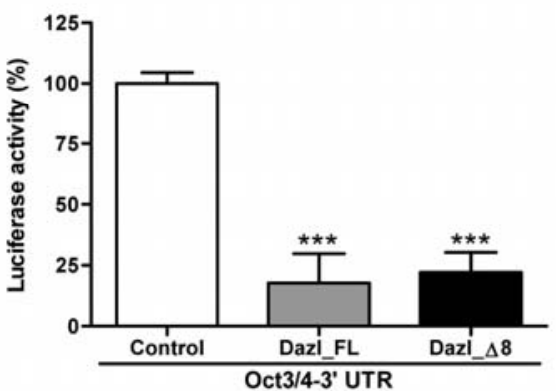

E.

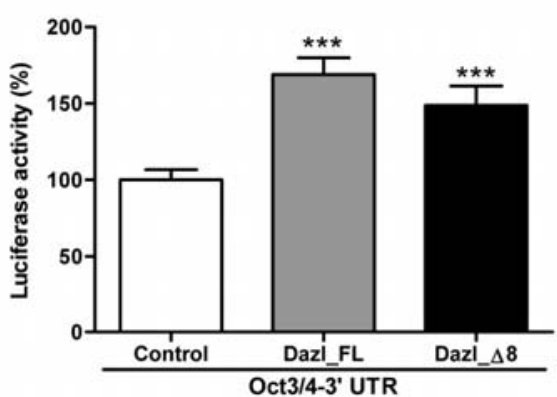

B.

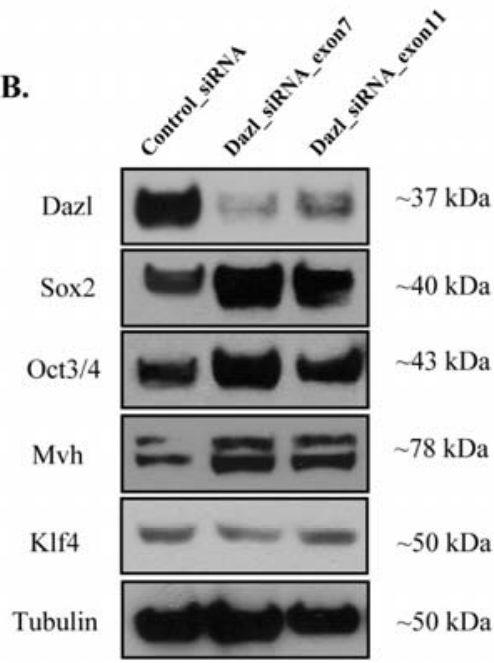

D.

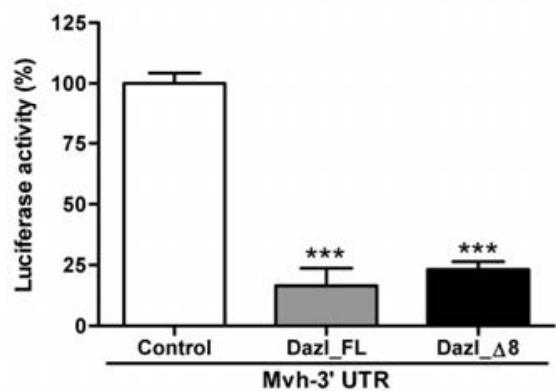

F.

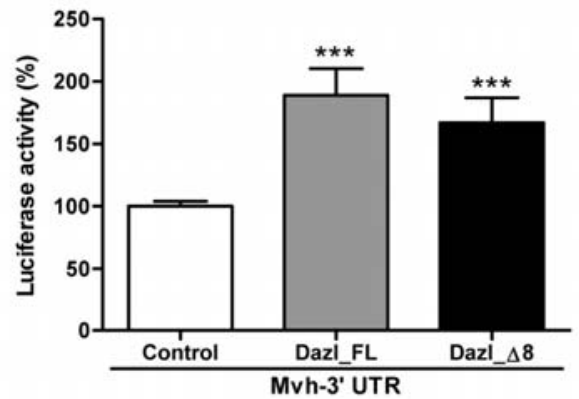

Figure 4. Dazl represses the translation of known target mRNAs in embryonic stem cells (ESCs). (A) Western blots showing the expression of Dazl, Sox2, Oct3/4, and Mvh upon either Dazl_FL-E2 or Dazl_ $\Delta 8$-E2 overexpression in ESCs. KIf4 was served as a control. All the blots were re-probed with tubulin antibody to verify equal protein loading and one representative blot is shown. (B) Western blots showing the protein expression of Dazl, Sox2, Oct3/4, and Mvh upon Dazl down-regulation using two independent siRNA oligonucleotides (Dazl_siRNA_exon7 and Dazl_siRNA_exon11). Transfection of control_siRNA (scrambled siRNA) into ESCs was used as a control. Klf4 was served as a control. All the blots were re-probed with tubulin and one representative blot is shown. (C) Bar graph showing the luciferase reporter activity of Oct3/4-3'UTR 
construct (pMIR-Oct3/4-3'UTR) in control (empty vector) and either Dazl_FL-E2 or Dazl_ $\Delta 8$ E2 overexpressing ESCs. (D) Bar graph showing the luciferase reporter activity of the $M v h-3$ 'UTR construct (pMIR-Mvh-3'UTR) in control (empty vector) and either Dazl_FL-E2 or Dazl_ $\Delta 8$ E2 overexpressing ESCs. (E) Bar graph showing the luciferase reporter activity of the Oct3/4-3'UTR construct (pMIR-Oct3/4-3'UTR) in control (empty vector) and either Dazl_FL-E2 or Dazl_ $\Delta 8$ _E2 overexpressing GC-1 cells. (F) Bar graph showing the luciferase reporter activity of the $M v h-3$ 'UTR construct (pMIR-Mvh-3'UTR) in control (empty vector) and either Dazl_FL-E2 or Dazl_ $\Delta 8 \_E 2$ overexpressing GC-1 cells. The error bar represents the mean \pm SD and the values that are statistically significant $(* * * \mathrm{p}<0.001)$ are indicated.

Dazl_siRNA treated cells compared to control_siRNA treated cells and found no significant differences (Supplementary Fig. S3B). To confirm the translational repressive role of Dazl, we performed luciferase reporter assays using constructs containing the 3'UTR of Oct3/4 (pMIR-Oct3/4-3'UTR) and the $3^{\prime}$ UTR of $M v h$ (pMIR-Mvh-3'UTR), which are cloned downstream of luciferase ORF into pMIR vector system. The co-expression of Dazl_FL-E2 and pMIR-Oct3/4-3'UTR in ESCs resulted in a significant decrease in the luciferase activity compared to empty vector and pMIR-Oct3/4-3'UTR transfected cells (Fig. 4C). Similarly, Dazl_A8-E2 expression also produced a significant reduction in luciferase reporter activity (Fig. 4C). Next, we co-expressed either Dazl_FL-E2 or Dazl_48-E2 together with the pMIR-Mvh-3'UTR construct in ESCs and found a very significant reduction in luciferase reporter activity, as observed for the pMIR-Oct3/4-3'UTR reporter (Fig. 4D). In addition, we performed luciferase reporter assays using GC-1 cell line and found dramatic increase in the luciferase activity of both $O c t 3 / 4$ and $M v h$ luciferase reporter constructs (Fig. 4E, F), confirming the translational stimulation role of Dazl in germ cells but not in ESCs.

\section{Identification of mRNAs associated with Dazl protein isoforms in ESCs}

Our studies on Dazl protein isoforms expression analysis and the translational regulation mechanisms in ESCs revealed high similarities between both Dazl_FL and Dazl_ $\Delta 8$ protein functions. To determine whether the Dazl_ $\Delta 8$ protein has any preferential mRNA targets in ESCs or rather binds and regulates only a subset of Dazl_FL mRNA targets, we transiently overexpressed each Dazl isoform (Dazl_FL-E2 and Dazl_A8-E2) separately in ESCs and performed RIP using E2-tag specific antibodies. Simultaneously, we also performed RIP with E2-tag antibodies using untransfected ESCs extract as a negative control, but failed to detect any RNA as measured on a Nanodrop spectrophotometer. Two independent RIPs were performed for each of Dazl isoform and the resulting RNA was independently hybridized to microarrays. The mRNA candidates enriched in each experiment were identified by intensity value in the array and the subsequent mean percentile rank assignment for two independent replicates of each Dazl isoform RIPs. The top ranked candidates of each Dazl isoform were selected based on $>0.98$ median percentile rank (Fig. $5 \mathrm{~A}, \mathrm{~B}$ ). Comparison of top ranked $(>0.98)$ candidates for Dazl_FL and Dazl_s8 revealed both isoform specific as well as common targets (Fig. 5C). Altogether, we identified 626 probes precipitated by the Dazl_FL isoform, of which 541 probes corresponding to 487 independent genes and 170 probes corresponding to unknown (NA) genes were specific for Dazl_FL (Fig. 5C). The remaining 85 probes corresponding to 66 individual genes and 16 probes corresponding to unknown were also precipitated by the Dazl_ $\Delta 8$ isoform, which were named common target mRNAs (Fig. 5C). Some of the known Dazl target mRNAs 
A.

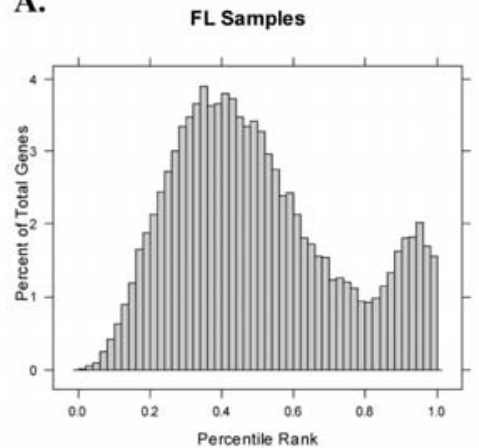

C.

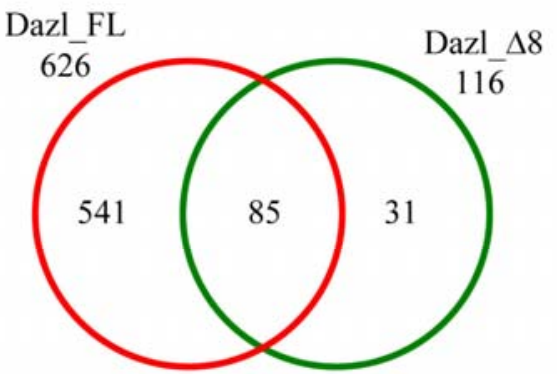

B.

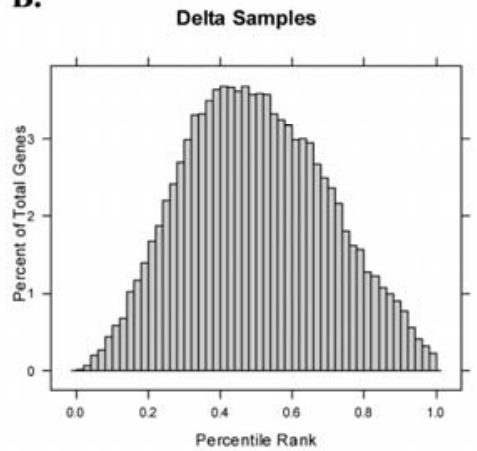

D.

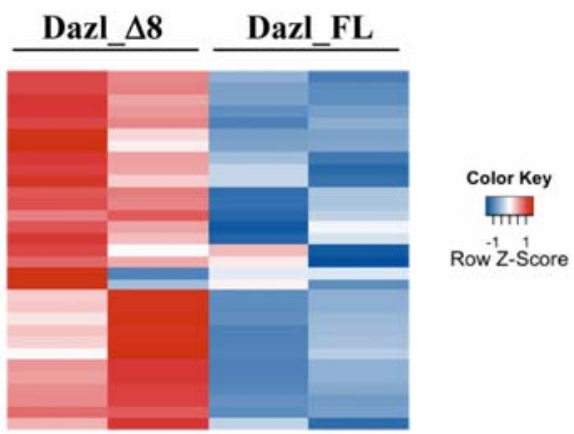

E.

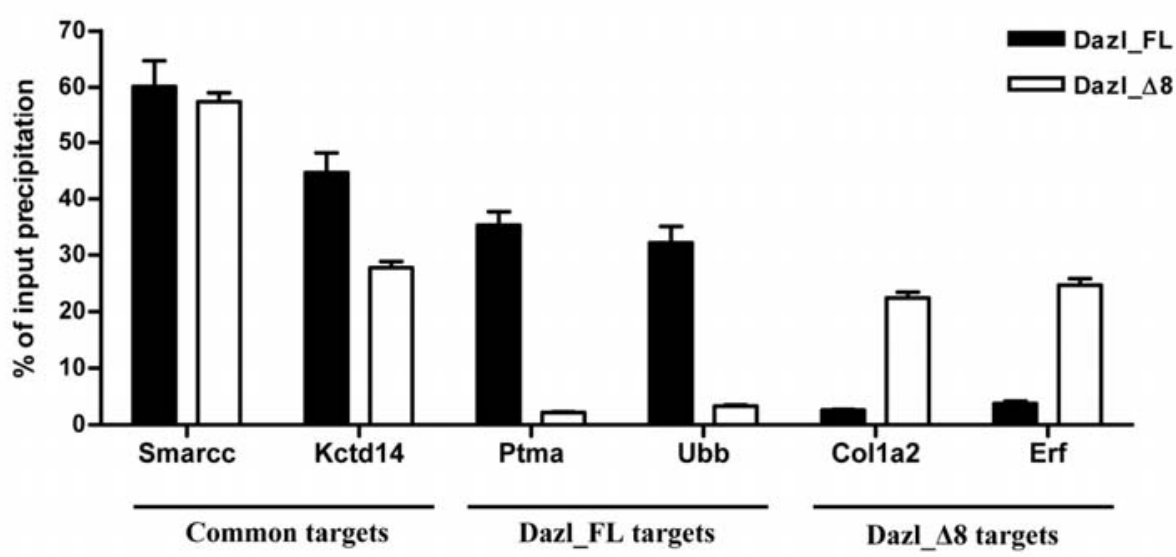

Figure 5. Identification and validation of Dazl-isoforms target mRNAs in embryonic stem cells (ESCs). Transcriptome analysis and subsequent percentile rank assignment image showing the distribution of the median percentile ranks for Dazl_FL (A) and Dazl_ $\Delta 8$ (B). (C) Venn diagram showing the number of common and isoform-specific target mRNAs between Dazl_FL and Dazl_ $\Delta 8$. (D) Heat map showing Dazl_ $\Delta 8$-specific target mRNAs (red-highly enriched) compared to Dazl_FL (blue-lower enriched). (E) Bar graph showing the qPCR validation of Dazl common (Smarcc and Ketd14) as well as Dazl_FL-specific (Ptma and Ubb) and Dazl_A8-specific (Col1a2 and Erf) targets mRNAs. 
such as Actg1, Calm2, and Slc2a3 (Reynolds et al., 2005) were readily detected in our array as Dazl_FL specific targets, confirming the specificity of our array (Table S1). For the Dazl_ $\Delta 8$ isoform, we identified 116 probes, of which 31 probes corresponding to 24 genes and 7 corresponding to unknown genes were specific for Dazl_ $\Delta 8$, while the remaining 85 probes/genes were common targets of both Dazl isoforms (Fig. 5C, D). A list of both common and isoform-specific target mRNAs is listed in supplementary Table S1. The transcriptome results were further confirmed by real-time PCR analysis for three selected candidates from each group (common, Dazl_FL-specific and Dazl_s8-specific) (Fig. 5E)

The Gene Ontology (GO) classification of common targets of Dazl isoforms revealed them as structural constituent of ribosome and translation regulators (Supplementary Table S7). Similarly, many Dazl_FL targets were classified as being involved in ribosome assembly and translation regulation. In contrast to these observations, the Dazl_ $\Delta 8$ isoform targets were identified as being involved in homeostasis processes such as cellular chemical, and ion homeostasis (Supplementary Table S7).

\section{Discussion}

Several lines of evidence suggest that the germ cell specific translational regulator Dazl is expressed in all pluripotent cell types and may play a key role in germ cell specification and differentiation [19, 20]. From Western blot analysis with a Dazl specific antibody on lysates from pluripotent cell types, we identified an additional band apart from the expected Dazl_FL. Our subsequent RT-PCR studies identified a novel splice variant of mouse Dazl (Dazl_48), which was expressed in the adult mouse testis, ovary, and also in all analyzed pluripotent cell types, albeit at low levels compared to Dazl_FL. Alternative splicing of Dazl exon8 was found to lead to the synthesis of protein lacking $17 \mathrm{aa}$ in the PR domain, while the rest of the protein architecture is intact and in-frame relative to Dazl_FL. We suggest that the additional band that we identified in Western blots is the Dazl_ $\Delta 8$ protein isoform, as we could also observe a reduced expression of this protein during downregulation experiments. Further support for this suggestion comes from our BNPS-Skatole cleavage experiments, where we could see two Dazl antibody reactive bands with an expected molecular weight difference. Generally, the PR regions are known for their role in mediating protein-protein interactions [42] and, indeed, the PR region consisting of a DAZ domain in the Dazl protein has been shown to mediate the interaction with several known interaction partners [43]. Recently it was proposed that the interrelated functions of Poly (A) binding family proteins, whose direct binding to Dazl regulate the translation [22], could be attributable to splice variants with specific deletion of proline-rich region [44]. However, the functional significance of such splice variants is currently unknown. Based on the above observations, we hypothesize that the specific deletion in the PR region of Dazl might mediate or impede specific protein interactions, thereby altering the target mRNA specificity.

In ESCs, the transcript levels of Dazl_ 48 were almost equal to those of Dazl_FL, whereas other pluripotent cell types had significantly lower levels of Dazl_48. For instance, maGSCs had significantly lower transcript levels of Dazl_48 compared to Dazl_FL. Interestingly, all pluripotent cell types with low levels of Dazl_ 48 originate from the germ-line stem cells, except for iPSCs; therefore, this might have some functional relevance for germ cell lineage. Dazl is expressed in oocytes and in the early stages of embryonic development $[2,17]$, as well as in the early and late germ-cell developmental stages [10, 16]. To delineate the expression of Dazl_48 in mouse testis development, as well as in the ovary, we performed qRT-PCR and detected the presence of the Dazl_48 transcript in all analyzed testis developmental stages and also the adult ovary. The 
transcript levels of Dazl_ $\Delta 8$ were almost half of those of Dazl_FL in both adult mouse testis and at any given developmental stage. These observations, together with the Dazl_48 transcript levels in pluripotent cells of germ-line origin further supports the possibility that there is functional relevance for these differences. The most widely used mouse testis mutants with defects at various stages of spermatogenesis such as $W / W^{V}, T f m / y$, leyI-l(-/-), olt/olt and $q k / q k$ offer the advantage of detecting the expression of a specific gene during various stages of spermatogenesis. Expression analysis of Dazl_ 48 in these mouse mutants revealed specific expression in all germ stem cells and their descending populations, but not in somatic cells of the testis. Collectively, these results argue for a conserved role for the Dazl_ $\Delta 8$ isoform along with the Dazl_FL protein, in both male and female mouse gametogenesis.

Dazl is a cytoplasmic protein with diffuse cytoplasmic protein localization, as well as being localized to stress granules, under both native and ectopically expressed conditions [41, 45]. Consistent with these reports, the ectopic expression of C-terminally tagged Dazl_FL protein had both a diffuse cytoplasmic and a large granular expression pattern. Intriguingly, the novel Dazl isoform, Dazl_ $\Delta 8$ also displayed both cytoplasmic and large granular localization, indicating that both Dazl isoforms might have overlapping functions.

The first evidence for the role of DAZ family proteins in translation regulation comes from genetic interaction studies in Drosophila, where twine was identified as a target of post-transcriptional regulation by boule [46]. Subsequent studies in mouse and Zebrafish identified the Dazl protein on actively translating polysomes and uncovered a role in translational stimulation of target mRNAs [47, 48]. Contrary to the known translational stimulatory role for Dazl in germ cells, we observed a translational repression role for Dazl in ESCs, and indeed, the Dazl known target $M v h$ was negatively regulated by both Dazl isoforms. Surprisingly, we also observed a negative translation regulation for Oct $3 / 4$ and Sox2, two well-characterized pluripotency marker genes. Our findings are in agreement with a previous study, which reported the translational repression of Tex19.1 mRNA by Dazl in Zebrafish embryos [49]. It is interesting to note that human hDAZ-associated protein 1 (DAZAP-1), a protein thought to function in translational repression, interacts with $\mathrm{hDAZ}$, thus, competing for the interaction with translation promoting factor, PABP [50]. These observations led to the suggestion that the binding of DAZAP-1 to DAZ might modulate or repress translation [51]. Moreover, hDAZL is known to interact with Pumilio-2, a well-known translational repressor, and suggested to function as translational regulator in ESCs and germ cells [18]. In light of these observations it is possible that the competition between Dazl interacting translation activator and repressor proteins in ESCs might render both translational activation and repression functions of Dazl, respectively. Alternatively, Dazl mediated mRNA stability might be important for changes in the target mRNA translation in a cell-type dependent manner. Recently, it was shown that Dazl plays a crucial role in the formation of SGs during heat induced stress conditions in male germ cells [45]. These observations led to a suggestion that the formation and the function of SGs during stress conditions are to repress translation initiation and to activate protective mechanisms in male germ cells $[41,45]$. In this context, the association of Dazl with SGs is interesting and we assume that Dazl might be important for the observed translational repression in SGs. Interestingly, the persistent expression of pluripotency marker genes such as Oct3/4 and Nanog were reported in Dazl-deficient germ cells, suggesting that Dazl might regulate the stem cell characteristics and differentiation [52]. Taken together, it is interesting to speculate that the physiological role of Dazl is to suppress the translation of germ cell specific transcripts in pluripotent cells. 
Research over the last decade has led to the identification of several mRNA targets and conserved sequence elements in the $3^{\prime}$ UTRs that are bound by Dazl protein [24, 25, 46, 47, 49, 53, 54]. The discovery of the Dazl_ $\Delta 8$ isoform prompted us to investigate the specific mRNA targets of this isoform, as well as to identify Dazl_FL target mRNAs in ESCs. Our immunoprecipitation-coupled transcriptome analysis revealed several target mRNAs that are bound by the Dazl isoforms, either selectively or in common. Interestingly, several of these target mRNAs (Actg1, Calm2, and Slc2a3) have already been described as putative targets of Dazl in germ cells [25], confirming the reliability of the method employed in the current study. Moreover, we identified specific target mRNAs for both Dazl isoforms in ESCs, suggesting functional divergence of these two isoforms. However, further studies are necessary to validate and dissect the interaction of Dazl isoforms with their target mRNAs and the functional significance of Dazl mediated translational regulation in ESCs. Unfortunately, our current RIP based transcriptome analysis failed to detect $M v h$, a known target of Dazl, for which we observed the translational repression by Dazl. Hence, it is possible that our present study could not detect all Dazl target mRNAs, this might be due to the sensitivity of RIP assay and the strength between mRNA and protein interaction. Alternatively, the observed translational repression by Dazl, at least for Oct $3 / 4$ and Sox2, might be an indirect effect, as some of Dazl interaction proteins can bind RNA and function as translational repressors. The GO analysis clearly indicated that both common and Dazl_FL targets as translation regulators, in line with the known function of Dazl in translation regulation. The identification of Dazl_ $\Delta 8$ putative targets as cellular homeostasis regulators is quite unexpected and requires further validation of these targets to delineate Dazl_ $\Delta 8$ function in these processes.

\section{Conclusions}

In summary, our current study describes the identification of a novel splice variant of Dazl (Dazl_48) and its expression in pluripotent cells, as well as in both testis and ovary. Further, we uncovered a translational repressive role for Dazl in ESCs, as opposed to the well-known translation stimulation function in germ cells. Finally, we have identified several putative target mRNAs that bound either specifically or by both Dazl isoforms in ESCs. Further studies aimed at understanding the role of Dazl_ $\Delta 8$ and their target mRNAs in pluripotent cells and in gametogenesis might uncover the functional significance of this novel protein isoform.

\section{Acknowledgments}

The authors would like to thank Dr. Gabriela Salinas-Riester for her great support with microarray experiments, Mr. Olaf Bernhard for his technical assistance and Dr. Jessica Nolte for valuable discussions. This work was partly supported by the German Research Foundation (Deutsche Forschungsgemeinschaft) DFG SPP1356 (EN 84/22-1) and DFG FOR 1041 (EN 84/23-1).

\section{References}

[1] E.S. Haag, Rolling back to BOULE, Proc Natl Acad Sci U S A, 98 (2001) 6983-6985.

[2] J.X. Peng, J.L. Xie, L. Zhou, Y.H. Hong, J.F. Gui, Evolutionary conservation of Dazl genomic organization and its continuous and dynamic distribution throughout germline development in gynogenetic gibel carp, J Exp Zool B Mol Dev Evol, 312 (2009) 855-871.

[3] R. Reijo, T.Y. Lee, P. Salo, R. Alagappan, L.G. Brown, M. Rosenberg, S. Rozen, T. Jaffe, D. Straus, O. Hovatta, et al., Diverse spermatogenic defects in humans caused by $\mathrm{Y}$ chromosome deletions encompassing a novel RNA-binding protein gene, Nat Genet, 10 (1995) 383-393.

[4] R. Saxena, L.G. Brown, T. Hawkins, R.K. Alagappan, H. Skaletsky, M.P. Reeve, R. Reijo, S. Rozen, M.B. Dinulos, C.M. Disteche, D.C. Page, The 
DAZ gene cluster on the human $\mathrm{Y}$ chromosome arose from an autosomal gene that was transposed, repeatedly amplified and pruned, Nat Genet, 14 (1996) 292-299.

[5] N. Reynolds, H.J. Cooke, Role of the DAZ genes in male fertility, Reprod Biomed Online, 10 (2005) 72-80.

[6] C.G. Eberhart, J.Z. Maines, S.A. Wasserman, Meiotic cell cycle requirement for a fly homologue of human Deleted in Azoospermia, Nature, 381 (1996) 783-785.

[7] D.W. Houston, M.L. King, A critical role for Xdazl, a germ plasm-localized RNA, in the differentiation of primordial germ cells in Xenopus, Development, 127 (2000) 447-456.

[8] T. Karashima, A. Sugimoto, M. Yamamoto, Caenorhabditis elegans homologue of the human azoospermia factor DAZ is required for oogenesis but not for spermatogenesis, Development, 127 (2000) 1069-1079.

[9] M. Ruggiu, R. Speed, M. Taggart, S.J. McKay, F. Kilanowski, P. Saunders, J. Dorin, H.J. Cooke, The mouse Dazla gene encodes a cytoplasmic protein essential for gametogenesis, Nature, 389 (1997) 73-77.

[10] R.A. Reijo, D.M. Dorfman, R. Slee, A.A. Renshaw, K.R. Loughlin, H. Cooke, D.C. Page, DAZ family proteins exist throughout male germ cell development and transit from nucleus to cytoplasm at meiosis in humans and mice, Biol Reprod, 63 (2000) 1490-1496.

[11] Y. Lin, D.C. Page, Dazl deficiency leads to embryonic arrest of germ cell development in XY C57BL/6 mice, Dev Biol, 288 (2005) 309-316.

[12] P.T. Saunders, J.M. Turner, M. Ruggiu, M. Taggart, P.S. Burgoyne, D. Elliott, H.J. Cooke, Absence of mDazl produces a final block on germ cell development at meiosis, Reproduction, 126 (2003) 589-597.

[13] J.Y. Tung, M.P. Rosen, L.M. Nelson, P.J. Turek, J.S. Witte, D.W. Cramer, M.I. Cedars, R.A. Pera, Variants in Deleted in AZoospermia-Like (DAZL) are correlated with reproductive parameters in men and women, Hum Genet, 118 (2006) 730-740.

[14] J.Y. Tung, M.P. Rosen, L.M. Nelson, P.J. Turek, J.S. Witte, D.W. Cramer, M.I. Cedars, R.A. Reijo-Pera, Novel missense mutations of the Deleted-in-AZoospermia-Like (DAZL) gene in infertile women and men, Reprod Biol Endocrinol, 4 (2006) 40.

[15] B.H. Schrans-Stassen, P.T. Saunders, H.J. Cooke, D.G. de Rooij, Nature of the spermatogenic arrest in Dazl -/- mice, Biol Reprod, 65 (2001) 771-776.

[16] K.M. Haston, J.Y. Tung, R.A. Reijo Pera, Dazl functions in maintenance of pluripotency and genetic and epigenetic programs of differentiation in mouse primordial germ cells in vivo and in vitro, PLoS One, 4 (2009) e5654.

[17] G. Cauffman, H. Van de Velde, I. Liebaers, A. Van Steirteghem, DAZL expression in human oocytes, preimplantation embryos and embryonic stem cells, Mol Hum Reprod, 11 (2005) 405-411.

[18] F.L. Moore, J. Jaruzelska, D.M. Dorfman, R.A. Reijo-Pera, Identification of a novel gene, DZIP (DAZ-interacting protein), that encodes a protein that interacts with DAZ (deleted in azoospermia) and is expressed in embryonic stem cells and germ cells, Genomics, 83 (2004) 834-843.

[19] X. Xu, D.V. Pantakani, S. Luhrig, X. Tan, T. Khromov, J. Nolte, R. Dressel, U. Zechner, W. Engel, Stage-specific germ-cell marker genes are expressed in all mouse pluripotent cell types and emerge early during induced pluripotency, PLoS One, 6 (2011) e22413.

[20] Z. Yu, P. Ji, J. Cao, S. Zhu, Y. Li, L. Zheng, X. Chen, L. Feng, Dazl promotes germ cell differentiation from embryonic stem cells, J Mol Cell Biol, 1 (2009) 93-103.

[21] J. Chen, C. Melton, N. Suh, J.S. Oh, K. Horner, F. Xie, C. Sette, R. Blelloch, M. Conti, Genome-wide analysis of translation reveals a critical role for deleted in azoospermia-like (Dazl) at the 
oocyte-to-zygote transition, Genes Dev, 25 (2011) 755-766.

[22] B. Collier, B. Gorgoni, C. Loveridge, H.J. Cooke, N.K. Gray, The DAZL family proteins are PABP-binding proteins that regulate translation in germ cells, Embo J, 24 (2005) 2656-2666.

[23] X. Jiao, P. Trifillis, M. Kiledjian, Identification of target messenger RNA substrates for the murine deleted in azoospermia-like RNA-binding protein, Biol Reprod, 66 (2002) 475-485.

[24] N. Reynolds, B. Collier, V. Bingham, N.K. Gray, H.J. Cooke, Translation of the synaptonemal complex component Sycp3 is enhanced in vivo by the germ cell specific regulator Dazl, Rna, 13 (2007) 974-981.

[25] N. Reynolds, B. Collier, K. Maratou, V. Bingham, R.M. Speed, M. Taggart, C.A. Semple, N.K. Gray, H.J. Cooke, Dazl binds in vivo to specific transcripts and can regulate the pre-meiotic translation of Mvh in germ cells, Hum Mol Genet, 14 (2005) 3899-3909.

[26] M. Zeng, W. Deng, X. Wang, W. Qiu, Y. Liu, H. Sun, D. Tao, S. Zhang, Y. Ma, DAZL binds to the transcripts of several Tssk genes in germ cells, BMB Rep, 41 (2008) 300-304.

[27] A.J. Matlin, F. Clark, C.W. Smith, Understanding alternative splicing: towards a cellular code, Nat Rev Mol Cell Biol, 6 (2005) 386-398.

[28] S. Stamm, S. Ben-Ari, I. Rafalska, Y. Tang, Z. Zhang, D. Toiber, T.A. Thanaraj, H. Soreq, Function of alternative splicing, Gene, 344 (2005) 1-20.

[29] J.L. Coulombre, E.S. Russel, Analysis of the pleiotropism at the $\mathrm{W}$-locus in the mouse; the effect of $\mathrm{W}$ and $\mathrm{W}$ substitution upon postnatal development of germ cells, Journal of Experimental Zoology, 126 (1954) 277-296.

[30] M.F. Lyon, S.G. Hawkes, X-linked gene for testicular feminization in the mouse, Nature, 227 (1970) 1217-1219.

[31] R. Moutier, New mutation causing sterility restricted to the male in rats and mice. In The Laboratory Animal in the Study of Reproduction,
The Laboratory Animal in the Study of Reproduction, (1976) 115-117.

[32] W.I. Bennett, A.M. Gall, J.L. Southard, R.L. Sidman, Abnormal spermiogenesis in quaking, a myelin-deficient mutant mouse, Biol Reprod, 5 (1971) $30-58$.

[33] S. Zimmermann, G. Steding, J.M. Emmen, A.O. Brinkmann, K. Nayernia, A.F. Holstein, W. Engel, I.M. Adham, Targeted disruption of the Insl3 gene causes bilateral cryptorchidism, Mol Endocrinol, 13 (1999) 681-691.

[34] J.H. Lee, D. Schutte, G. Wulf, L. Fuzesi, H.J. Radzun, S. Schweyer, W. Engel, K. Nayernia, Stem-cell protein Piwil2 is widely expressed in tumors and inhibits apoptosis through activation of Stat3/Bcl-XL pathway, Hum Mol Genet, 15 (2006) 201-211.

[35] T. Khromov, D.V. Pantakani, J. Nolte, M. Wolf, R. Dressel, W. Engel, U. Zechner, Global and gene-specific histone modification profiles of mouse multipotent adult germline stem cells, Mol Hum Reprod, 17 (2011) 166-174.

[36] L. Smorag, Y. Zheng, J. Nolte, U. Zechner, W. Engel, D.V. Pantakani, MicroRNA signature in various cell types of mouse spermatogenesis: Evidence for stage-specifically expressed miRNA-221, -203, and -34b-5p mediated spermatogenesis regulation, Biol Cell, (2012).

[37] S. Meyer, J. Nolte, L. Opitz, G. Salinas-Riester, W. Engel, Pluripotent embryonic stem cells and multipotent adult germline stem cells reveal similar transcriptomes including pluripotency-related genes, Mol Hum Reprod, 16 (2010) 846-855.

[38] W.H. Townley-Tilson, S.A. Pendergrass, W.F. Marzluff, M.L. Whitfield, Genome-wide analysis of mRNAs bound to the histone stem-loop binding protein, Rna, 12 (2006) 1853-1867.

[39] C. Schulz, O. Lytovchenko, J. Melin, A. Chacinska, B. Guiard, P. Neumann, R. Ficner, O. Jahn, B. Schmidt, P. Rehling, Tim50's presequence receptor domain is essential for signal driven 
transport across the TIM23 complex, J Cell Biol, 195 643-656.

[40] D.L. Crimmins, S.M. Mische, N.D. Denslow, Chemical cleavage of proteins in solution, Curr Protoc Protein Sci, Chapter 11 (2005) Unit 1114.

[41] K.H. Lee, S. Lee, B. Kim, S. Chang, S.W. Kim, J.S. Paick, K. Rhee, Dazl can bind to dynein motor complex and may play a role in transport of specific mRNAs, Embo J, 25 (2006) 4263-4270.

[42] M.P. Williamson, The structure and function of proline-rich regions in proteins, Biochem J, 297 (Pt 2) (1994) 249-260.

[43] S. Tsui, T. Dai, S. Roettger, W. Schempp, E.C. Salido, P.H. Yen, Identification of two novel proteins that interact with germ-cell-specific RNA-binding proteins DAZ and DAZL1, Genomics, 65 (2000) 266-273.

[44] B. Gorgoni, N.K. Gray, The roles of cytoplasmic poly(A)-binding proteins in regulating gene expression: a developmental perspective, Brief Funct Genomic Proteomic, 3 (2004) 125-141.

[45] B. Kim, H.J. Cooke, K. Rhee, DAZL is essential for stress granule formation implicated in germ cell survival upon heat stress, Development, 139 (2012) 568-578.

[46] J.Z. Maines, S.A. Wasserman, Post-transcriptional regulation of the meiotic $\mathrm{Cdc} 25$ protein Twine by the Dazl orthologue Boule, Nat Cell Biol, 1 (1999) 171-174.

[47] S. Maegawa, M. Yamashita, K. Yasuda, K. Inoue, Zebrafish DAZ-like protein controls translation via the sequence 'GUUC', Genes Cells, 7 (2002) 971-984.
[48] S. Tsui, T. Dai, S.T. Warren, E.C. Salido, P.H. Yen, Association of the mouse infertility factor DAZL1 with actively translating polyribosomes, Biol Reprod, 62 (2000) 1655-1660.

[49] M. Zeng, Y. Lu, X. Liao, D. Li, H. Sun, S. Liang, S. Zhang, Y. Ma, Z. Yang, DAZL binds to 3'UTR of Tex19.1 mRNAs and regulates Tex19.1 expression, Mol Biol Rep, 36 (2009) 2399-2403.

[50] S. Morton, H.T. Yang, N. Moleleki, D.G. Campbell, P. Cohen, S. Rousseau, Phosphorylation of the ARE-binding protein DAZAP1 by ERK2 induces its dissociation from DAZ, Biochem J, 399 (2006) 265-273.

[51] M. Brook, J.W. Smith, N.K. Gray, The DAZL and PABP families: RNA-binding proteins with interrelated roles in translational control in oocytes, Reproduction, 137 (2009) 595-617.

[52] M.E. Gill, Y.C. Hu, Y. Lin, D.C. Page, Licensing of gametogenesis, dependent on RNA binding protein DAZL, as a gateway to sexual differentiation of fetal germ cells, Proc Natl Acad Sci U S A, 108 (2011) 7443-7448.

[53] M. Fox, J. Urano, R.A. Reijo Pera, Identification and characterization of RNA sequences to which human PUMILIO-2 (PUM2) and deleted in Azoospermia-like (DAZL) bind, Genomics, 85 (2005) 92-105.

[54] J.P. Venables, M. Ruggiu, H.J. Cooke, The RNA-binding specificity of the mouse Dazl protein, Nucleic Acids Res, 29 (2001) 2479-2483. 
Results

A.

Dazl_FL-specific primers standard curve

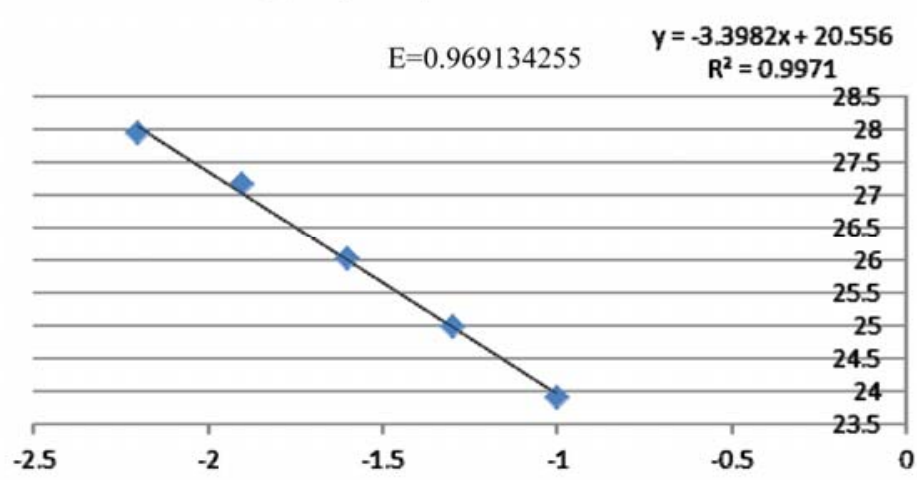

B.

Dazl_ $\Delta$ 8-specific primers standard curve

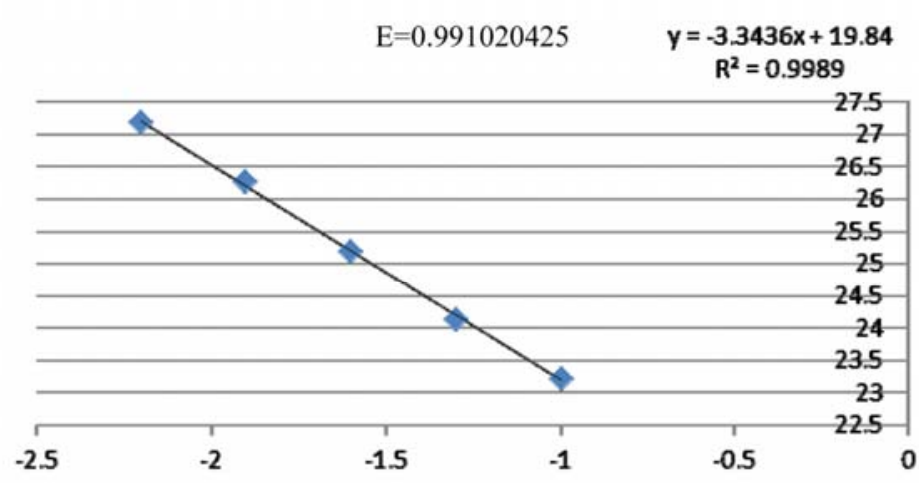

Figure S1. Amplification efficiency of Dazl isoform-specific primers. The standard curve graph showing the amplification efficiency of Dazl_FL-specific (A) and Dazl_ $\Delta 8$-specific (B) primer pairs. 


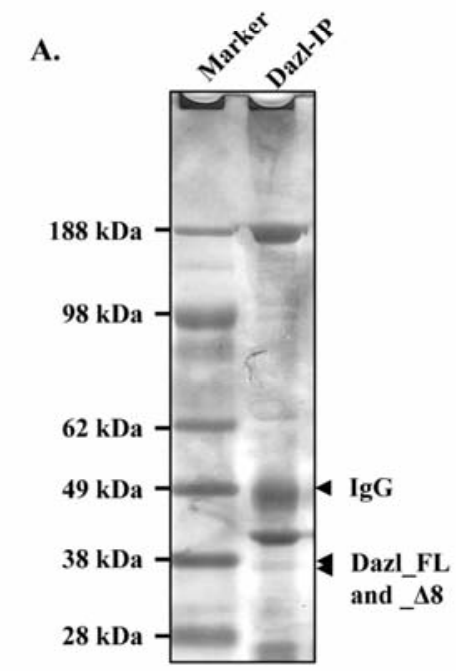

C.

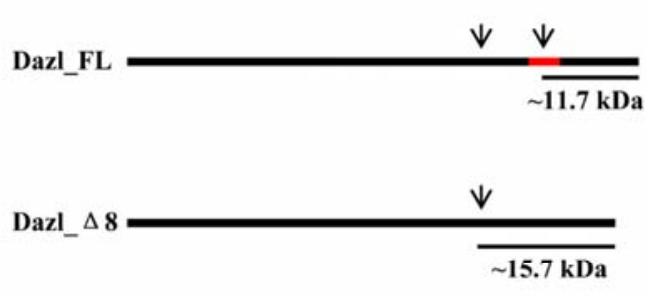

B.

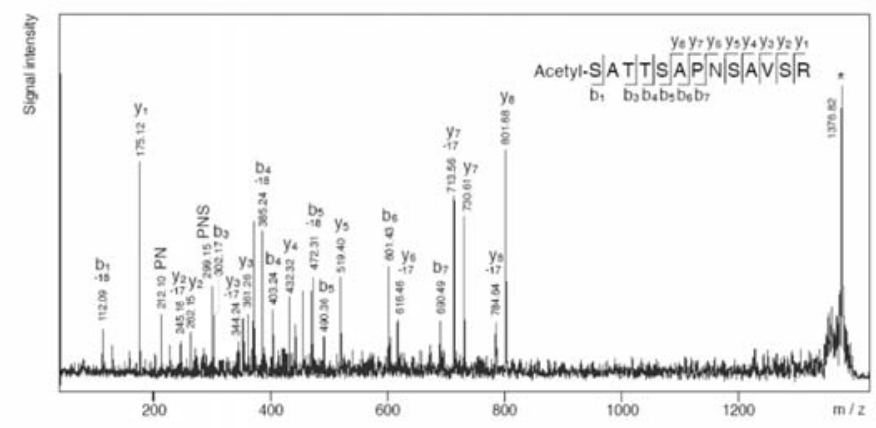

Figure S2. Biochemical characterization of Dazl protein isoforms. (A) Coomassie blue stained SDS-PAGE gel showing the Dazl-IPed proteins. The Dazl_FL and Dazl_ $\Delta 8$ protein isoforms as well as IgG-heavy chain are indicated with arrowheads. (B) The isoforms of Dazl (Dazl_FL + Dazl_ $\Delta 8$ ) purified by SDS-PAGE were subjected to tryptic in-gel digestion and LC-MALDI mass spectrometry. The fragment ion spectrum of the $\mathrm{N}$-terminal peptide (precursor mass 1419.67) is shown, which indicates that the $\mathrm{N}$-terminal amino acid sequence starts from serine at position 2 carrying an $\mathrm{N}$-terminal acetyl group. The $\mathrm{b}$ - and $\mathrm{y}$-ion series and internal fragments are indicated, the asterisk marks a fragment generated by the neutral loss of the acetyl group. (C) Schematic diagram showing the Dazl_FL and Dazl_A8 protein isoforms along with the BNPS-Skatole cleavage sites (arrows). Exon8 coding region is highlighted as red in Dazl_FL protein. The expected C-terminal protein fragments along with their predicted molecular weights are indicated below the each protein. (D) Western blot showing the detection of Dazl protein isoforms either in absence or presence of BNPS-Skatole. 
A.

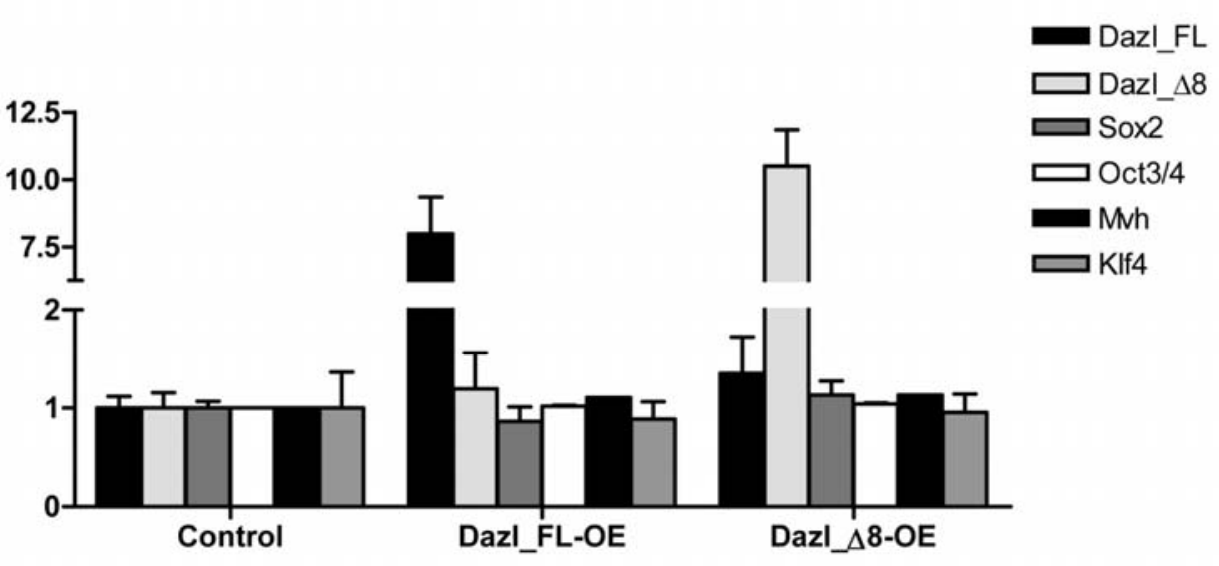

B.

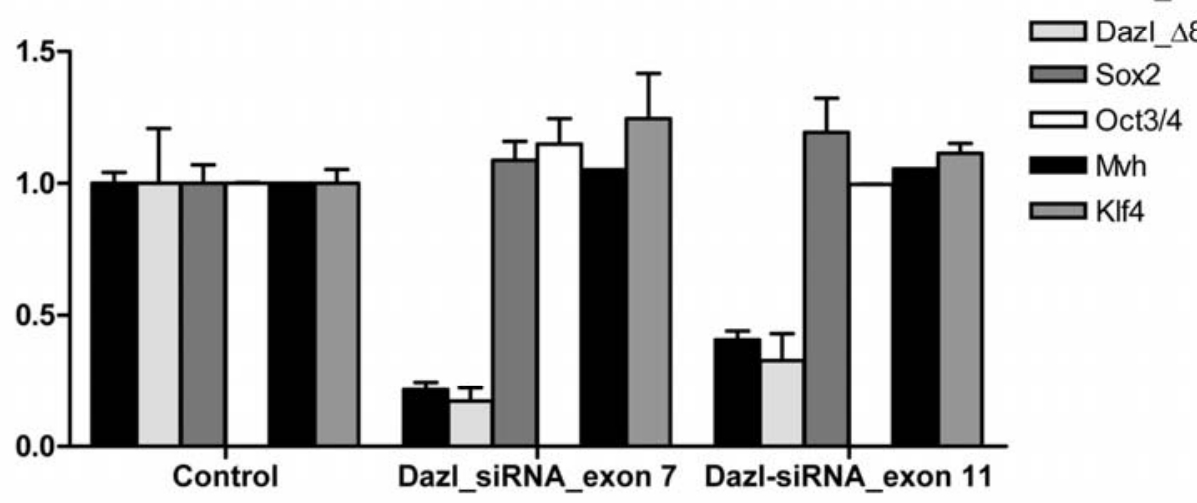

Figure S3. Alteration of Dazl expression has no effect on selected mRNA expression levels. qRT-PCR data showing the transcript levels of Dazl isoforms and target (Sox2, Oct3/4 and Mvh) and control (Klf4) mRNAs in control ESCs and Dazl splice variants overexpressing (Dazl_FL and Dazl_ $\left.\Delta 8 \_E 2\right)$ ESCs (A) and Dazl downregulated (Dazl_siRNA_exon7 and Dazl_siRNA_exon 11 ) ESCs

(B) . 


\section{Discussion}

Embryonic stem cells (ESCs) derived from the inner cell mass (ICM) of blastocysts are capable of differentiating into all cell types of the adult organism and are regarded as the "gold standard" of pluripotency (Smith et al., 2009; Bilic et al., 2012). These unique characteristics imply that the production of lineage-specific cell types differentiated from ESCs might be possibly applied in cell replacement therapies (Lerou and Daley, 2005). Therefore, they have become the focal point of a burgeoning field of biomedical research and regenerative medicine. Germ cells are the truly and unique immortal cells which are able to surpass the genetic information from one generation to the next, indefinitely (McLaren, 2001). Interestingly, germ stem cells, the unipotent stem cells, isolated at various stages of germ cell development have a unique ability to transform into pluripotent stem cells (Kahan et al., 1970; Matsui et al., 1992; Resnick et al., 1992; Guan et al., 2006; Kanatsu-Shinohara et al., 2004). Moreover, several studies have shown the expression of several germ cell marker genes in pluripotent stem cells including ESCs (Geijsen et al., 2004; Qing et al., 2007; Mise et al., 2008) and also reported their spontaneous differentiation towards germ cells (Hübner et al., 2003; Geijsen et al., 2004; LachamKaplan et al., 2006). These results highlight the possible reciprocal relationship between germ cells and pluripotent stem cells and even caste the doubt on the origin of ESCs.

In the present study, we systematically analyzed the expression of selected germ stem cell (GC) and premeiotic (PrM) marker genes in several pluripotent stem cells (Xu et al., 2011). Our results indicate that the expression of GC and PrM marker genes are important for the establishment of pluripotency and germ line commitment of pluripotent stem cells, respectively. Moreover, our findings implied that the expression of GC marker genes triggered by BMP signals parallels between in vivo germ cell specification and in vitro somatic cell reprogramming to induced pluripotency, indicating the establishment of pluripotency probably via the germ cell fate/origin. In line with these assumptions, our further results indicate that the expression of Dppa3, a GC marker gene, is important for the establishment of authentic pluripotency during somatic cell reprogramming, while the expression of Dazl, a PrM marker gene, can lead to the generation of putative germ cells.

\subsection{Spermatogenesis reconstituted from pluripotent stem cells in vitro}

Over $10 \%$ of couples in the world are suffering from infertility and the males are account for approximately $50 \%$ of all cases (Schlegel, 2009). Male infertility can be 
attributed by spermatogenic arrest at different stages of meiosis. Therefore, the derivation of haploid gametes ex vivo is being considered as an alternative approach to treat male infertility. Recent advances in germ cell research have shown the possibility to generate haploid germ cells using in vitro system (Hübner et al., 2003; Nayernia et al., 2006; Yu et al., 2009).

The derivation of haploid germ cells from various pluripotent stem cells has been established almost a decade ago. The first study reported the spontaneous differentiation of ESCs towards germ cell lineage under LIF and feeder depleted culture conditions (Hübner et al., 2003). Thereafter, several distinct strategies were also proved the possibility to obtain PGCs and even haploid gametes from ESCs by mimicking the in vivo environment in embryoid bodies (Toyooka et al., 2003; Geijsen et al., 2004; Lacham-Kaplan et al., 2006), by the activation of germ cell differentiation pathways using growth factors (Nayernia et al., 2006) or through overexpression of PrM marker genes ( $\mathrm{Yu}$ et al., 2009). This differentiation property towards germ cell lineage is not only restricted to ESCs but also demonstrated for other pluripotent stem cells such as embryonal carcinoma cells (ECCs) (Nayernia et al., 2004), embryonic germ cells (EGCs) (Eguizabal et al., 2009), multipotent adult germ line stem cells (maGSCs) (Nolte et al., 2010) and induced pluripotent stem cells (iPSCs) (Imamura et al., 2010; Zhu et al., 2012; Yang et al., 2012). In order to facilitate human reproductive medicine, these protocols were applied to human model and successfully produced primordial germ cells (PGCs) from human ESCs (Tilgner et al., 2008; West et al., 2008; Bucay et al., 2009; Aflatoonian et al., 2009) and human iPSCs (Park et al., 2009). Additionally, several groups have reported that human pluripotent stem cells can enter meiosis and produce haploid cells (Eguizabal et al., 2011; Panula et al., 2011). Thus, many reproducible results have highlighted the feasibility to produce germ cell or haploid cells from pluripotent cells. Hereby this in vivo system can be used as an excellent tool to study germ cell development and signalling processes in an easy to access model. Moreover, it holds the therapeutic potential for treatment of male infertility.

Strikingly, several of the above mentioned studies have noted the expression of some of the GC and PrM marker genes already in undifferentiated pluripotent cells (Geijsen et al., 2004; Qing et al., 2007; Mise et al., 2008). These observations led to a suggestion that ESCs, apart from germ line derived pluripotent stem cells, might have a germ cell origin or the expression of GC marker genes might be important for the establishment/maintenance of pluripotency. 


\subsection{Stage-specific germ cell marker genes are expressed in all mouse pluripotent cell types and emerge early during induced pluripotency}

Since the techniques for successful generation of ECCs and ESCs were established in the mouse, a series of pluripotent stem cell lines were established. Historically, the pluripotent EGCs, ECCs and maGSCs have been documented, all with germ line origins (Kahan et al., 1970; Matsui et al., 1992; Resnick et al., 1992; Guan et al., 2006; KanatsuShinohara et al., 2004). However, till now there is no convincing result to show either the ICM or the germ cell origin of ESCs. High similarities between germ cells and ESCs made stem cell researchers to speculate the possible germ cell origin of ESCs (Zwaka et al., 2005; Nichols and Smith 2011). Traditionally, ESCs are regarded as an in vitro equivalent of the ICM, but it was shown that ESCs more closely resemble cells derived from the primitive ectoderm (Gardner and Brook, 1997). Recently, two independent groups have shown that only a small subset of cells in the ICM, which are positive for GC marker genes, are able to give rise to ESCs in vitro (Nichols et al., 2009; Chu et al., 2011). The authors constructed a reporter system by fusing the promoter region of Blimp1, the GC determinant, to a fluorescent protein to monitor the signal during the process of ESC establishment. Notably, the authors observed the positive signal from 2 days after blastocyst culture; moreover, a clear outgrowth resembling ESC-like colony appeared only 4 days after the culture (Chu et al., 2011). As expected, the Blimp1 positive cells also showed expression of other GC marker genes such as Dppa3, Ifitm3, Prdm14 and Lin28 (Chu et al., 2011). This outcome reflected that there is an intermediate state of PGC-like property acquisition during the early derivation process of ESCs. In agreement with these results, our study (Xu et al., 2011) could show that the GC marker genes possess an active chromatin signature at their promoter regions in ESCs, which could be a reminiscent of PGC fate determination during early events of ESC establishment.

These observations raised the question whether there is also a temporary PGC fate during iPS cell generation. In our study (Xu et al., 2011), we attempted to answer this question by direct reprogramming of somatic cells into iPSCs and by analysing the activation of selected GC/PrM markers along with the activation of core pluripotency marker genes during this process. Intriguingly, the activation of GC markers Blimpl, Dppa3 and Ifitm3 was found to precede the activation of the endogenous pluripotency markers such as Oct3/4, Sox 2 and Nanog. In contrast, the expression of PrM marker genes such as Dazl, Mvh and Stra8 was detected only in established iPS cell lines. Meanwhile, two elegant studies revealed that Mesenchymal-to-Epithelial Transition (MET) is necessary 
during the initiation stage of reprogramming and this key event is driven by the BMP signalling pathway (Li et al., 2010; Samavarchi-Tehrani et al., 2010). Furthermore, all ESC-like characteristics are acquired by sequential expression of cell adherence marker genes $C d h 1$ and $\beta$-catenin, followed by concomitant upregulation of early pluripotency marker genes such as AP and SSEA1 (Samavarchi-Tehrani et al., 2010). By a coincidence, at early gastrulation of embryo development, PGC precursors are induced by an instructive BMP signalling followed by MET and the expression of GC marker genes Blimp1, Dppa3, Ifitm3 along with Cdh1 (Matsui, 2009; Saitou, 2009). Notably, loss of BMP signal leads to lack of PGCs in vivo and inactivation of BMP results in failure to generate iPSCs from somatic cells (Lawson et al., 1999; Saito, 2009). Collectively, through our expression analysis and observation of molecular parallels between PGC specification and iPSC generation, we speculate that even somatic cells have to pass through a temporary PGC fate to achieve pluripotency during reprogramming.

\subsubsection{Expansion of PGC fate model to human somatic cell reprogramming}

To assess whether the temporary PGC fate is also recapitulated during human somatic cell reprogramming, we decided to analyze the GC, PrM and pluripotency marker genes expression during reprogramming of adult human fibroblast cells. In the first pilot screening, we decided to collect one sample from each three distinct phases (initiation, maturation and stabilization) of reprogramming, i.e., at day 14, day 20, and day 30 as well as wild type fibroblast cells and established human induced pluripotent stem cells (hiPSCs) as negative and positive controls, respectively. Quantitative real time PCR analysis revealed a very high significant expression levels of GC markers (Blimpl and Ifitm3) already in wild type fibroblasts and these expression levels were gradually reduced and reached to levels seen in established iPSCs by day 30 (Fig. 4.1). In contrast, the expression of Dppa3, the other GC marker gene, was only detectable at day 20 and significantly increased to 100-fold than the expression levels of iPSCs (Fig. 4.1). The endogenous expression of key pluripotency markers Oct4, Sox 2 and Nanog appeared by day 20 and showed increased expression by day 30 (Fig. 4.1). Surprisingly, we could detect a significant expression of PrM marker gene Dazl on day 14 of reprogramming with an increased trend till day 30 (Fig. 4.1). Collectively, these preliminary results indicate that the expression pattern of GC and PrM marker genes before and during somatic reprogramming differ between mouse and human fibroblasts. In order to rule out that the expression of GC marker genes is specific to this particular fibroblast cell line which we 
used for reprogramming studies, we analyzed and found the expression of GC marker genes in 4 different human fibroblast cell lines (data not shown).
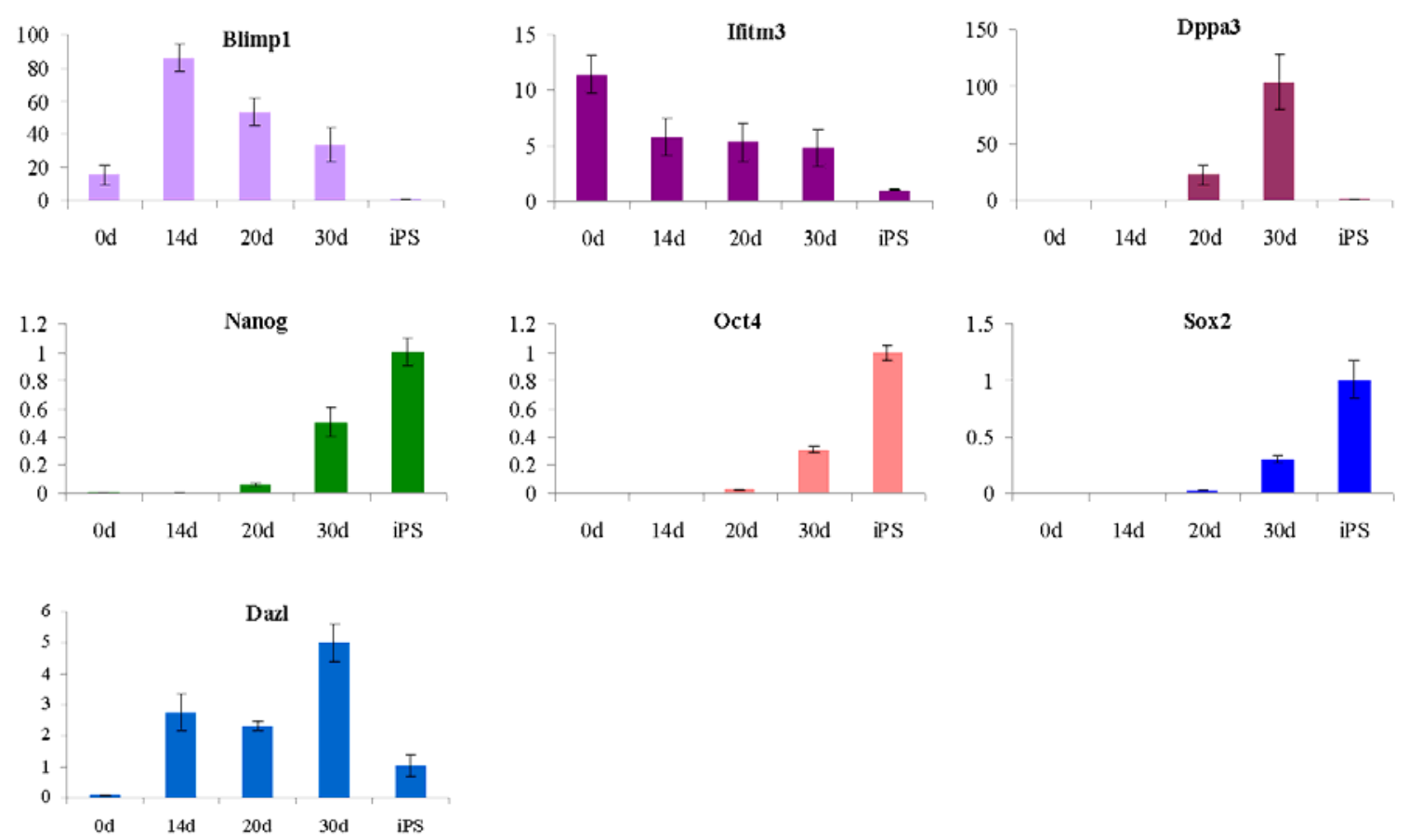

Figure 4.1. Expression pattern of GC/PrM- and pluripotency-marker genes during the course of hiPS generation. Quantitative real time qPCR analysis of germ stem cell (Blimp1, Ifitm3, and Dppa3), pluripotency (Oct3/4, Sox2, and Nanog), and pre-meiotic (Dazl) genes during the time course of iPS cell generation. 0d- wild type human fibroblasts; 14d-, 20d-, 30d- : day 14, day 20, and day30 after virus infection; iPS- established human iPS cells. The qPCR data of three technical replicates were calculated and represented as a mean $\pm \mathrm{SD}$.

Although human ESCs are broadly used as a model for biomedical research, they are more difficult to manipulate than their counter parts in mouse (Nichols and Smith, 2011). Two distinct groups of pluripotent cells are derived from mouse early embryonic development: (1) ESCs are established from E3.5- E4.5 blastocysts and (2) epiblast stem cells (EpiSCs) are derived from E5.5- E6.5 post implantation embryos (Evans and Kaufman, 1981; Brons et al., 2007; Tesar et al., 2007). ESCs are regarded to bear a ground state (naïve) of pluripotency; however, EpiSCs hold a "primed" state of pluripotency. Unlike ESCs, EpiSCs are not able to contribute to chimeras after their injection into blastocysts (Nichols and Smith, 2009). Interestingly, the high molecular similarities between human ESCs and mouse EpiSCs led to propose that human ESCs, in contrast to mouse ECS, are in a "primed" state of pluripotency (Nichols et al., 2009). Taken together, we speculate that there are fundamental differences between mouse and human ESCs and somatic reprogramming and this could be partly attributable to the cell intrinsic and species 
specific mechanisms. However, further studies are necessary to identify the PGC fate during human iPSC generation.

\subsubsection{Transdifferentiation of somatic cells into induced germline stem cells}

The observation that GC marker genes are expressed earlier than that of pluripotency marker genes and the possible temporary PGC fate during somatic cell reprogramming prompted us to evaluate the feasibility to derive germ line stem cells (GSCs) directly from somatic cells. At the meantime, it was shown that mouse ESCs and iPSCs are able to generate PGC-like cells through an epiblast-like state, and these cells have been demonstrated for their capability to initiate spermatogenesis (Hayashi et al., 2011). Based on these observations, we explored a condition in which fibroblast cells can transdifferentiate into germline stem cells.

In order to screen the candidate $\mathrm{GC}$ factor(s) that induces transdifferentiation of fibroblast cells to a germ cell state, we used OSKM (Oct3, Sox2, Klf4 and $c-M y c)$ in combination with GC marker genes (Blimp1, Ifitm3, Dppa3) for reprogramming studies as outlined in figure 4.2. Approximately after two weeks of transduction, few round and clustered cells were formed and resembled PGC/SSC-like morphology in the combination of OSKM+Blimp1 (OSKMB) (Fig. 4.3 A). In contrast, OSKM cells showed compacted ESC like colonies (Fig. 4.3 A). The induced GSCs (iGSCs) obtained in OSKMB combination were able to proliferate and showed typical grape-like morphology of germ cells and are positive for alkaline phosphatase $(A P)$ staining (Fig. 4.3 A).
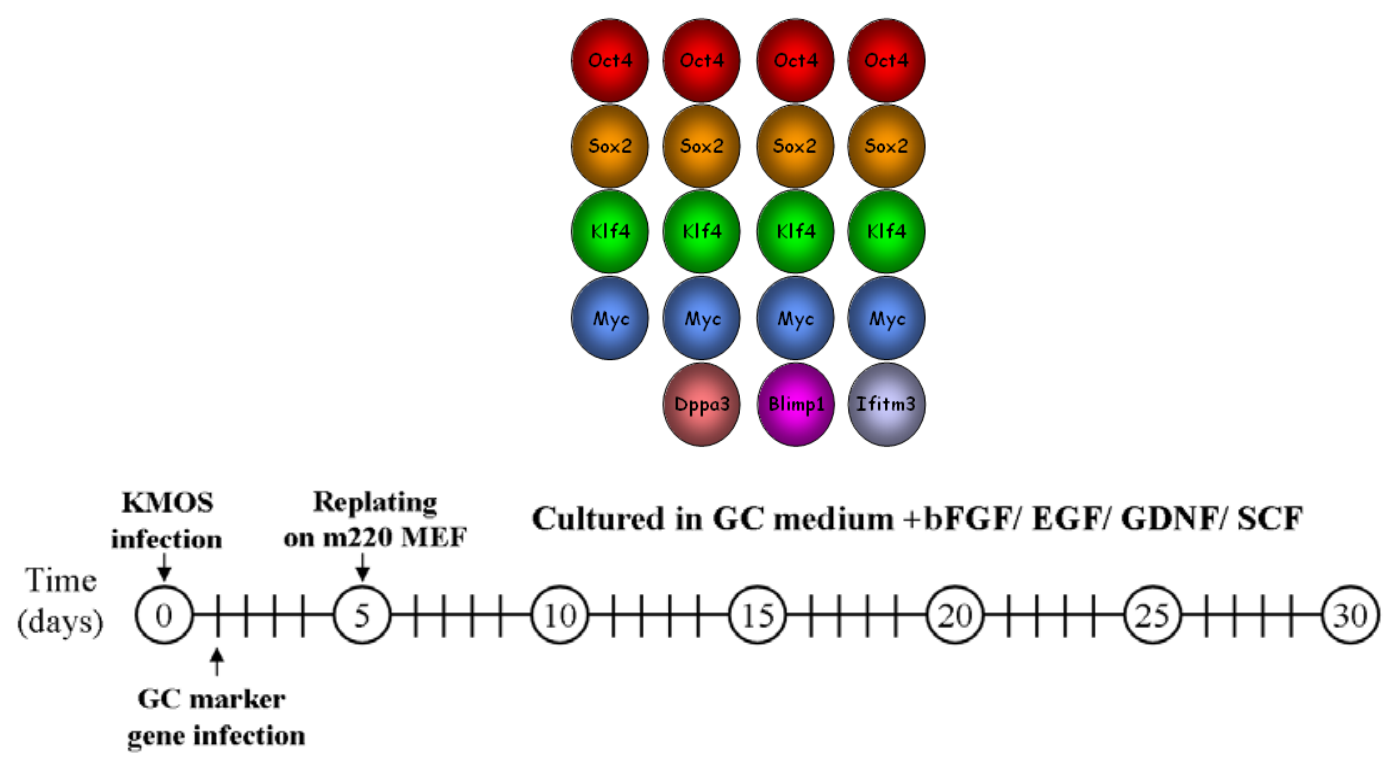

Figure 4.2. Schematic diagram depicting the generation of iGSCs. The iGSCs were generated by using different combinations of reprogramming factors along with GC factors and growth factors, which are supplemented to the medium to facilitate the generation of iGSCs. 
To further characterize the iGSCs, we performed RT-PCR analysis for the expression of GC marker genes Oct4, Cdh1, Plzf, and Gfro-1. These studies revealed that the expression of typical GC marker genes Plzf and Gfro-lare highly expressed in iGSCs and spermatogonial stem cells (SSCs), an established SSC line or testis, but not in ESCs (Fig. 4.3 B). In addition, these iGSCs were positive for Plzf and Gfr $\alpha-1$ expression as judged by immunocytochemistry (Fig. 4.4 A), however, the expression of pluripotent cell marker SSEA1 was visible only in ESCs but not in iGSCs and SSCs (Fig. 4.4 B).

A.
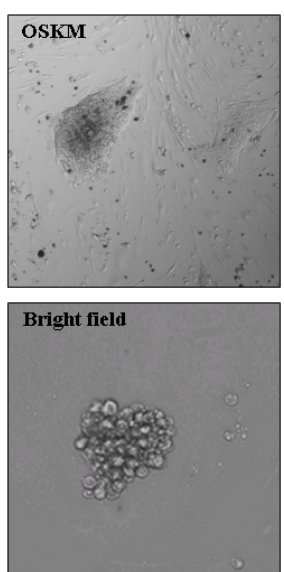
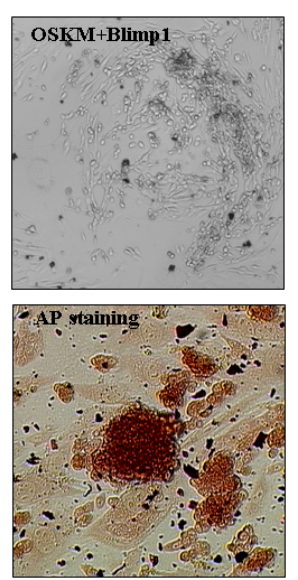

B.

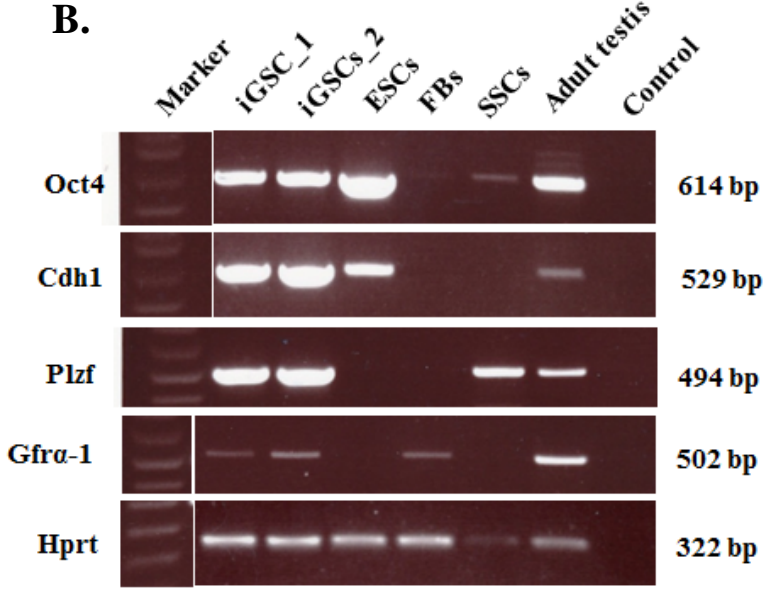

Figure 4.3. Generation and characterization of iGSCs. (A) Colony morphology of iPSCs generated with OSKM or iGSCs generated in the presence of OSKMB. (B) RT-PCR analysis for the expression of GC genes in two iGSC lines, ESCs, fibroblasts (FBs), SSCs, and WT- testis. Water (control) was used as a negative control.

To rule out that iGSCs are not a pluripotent cell type, we injected these cells into immunodeficient mice and observed a malignant tumour but not the teratoma, suggesting that these cells are not pluripotent. Next, to assess the functional properties of iGSCs in reconstitution of spermatogenesis in vivo, we transplanted these cells into the testis of busulfan treated mice. Unfortunately, these mice developed testicular tumours suggesting that the derivation of iGSCs protocol needs to be optimized. Collectively, in this part of our study, we developed an in vitro method that achieves three significant endpoints. First, the generation of PGC- or SSC-like cells from somatic cells is possible and it gives a strong evidence for our hypothesis that there is a temporary germ cell fate before somatic cells enter into the pluripotent state. Second, this is the first ever report that successfully generates putative germ cells directly from somatic cells without entering any intermediate cell stage(s). The mechanism of PGC development is difficult to study mainly because of the inaccessibility of PGC during development. Our PGC-like cell induction system thus might facilitate the understanding of this complicated process. Third, our results agree with 
those in a previous report, which showed that Blimp1 expression mediates the germ cell fate commitment (Ohinata et al., 2005). These data may therefore also have ramifications for reproductive technology as well as for disease modelling approaches.
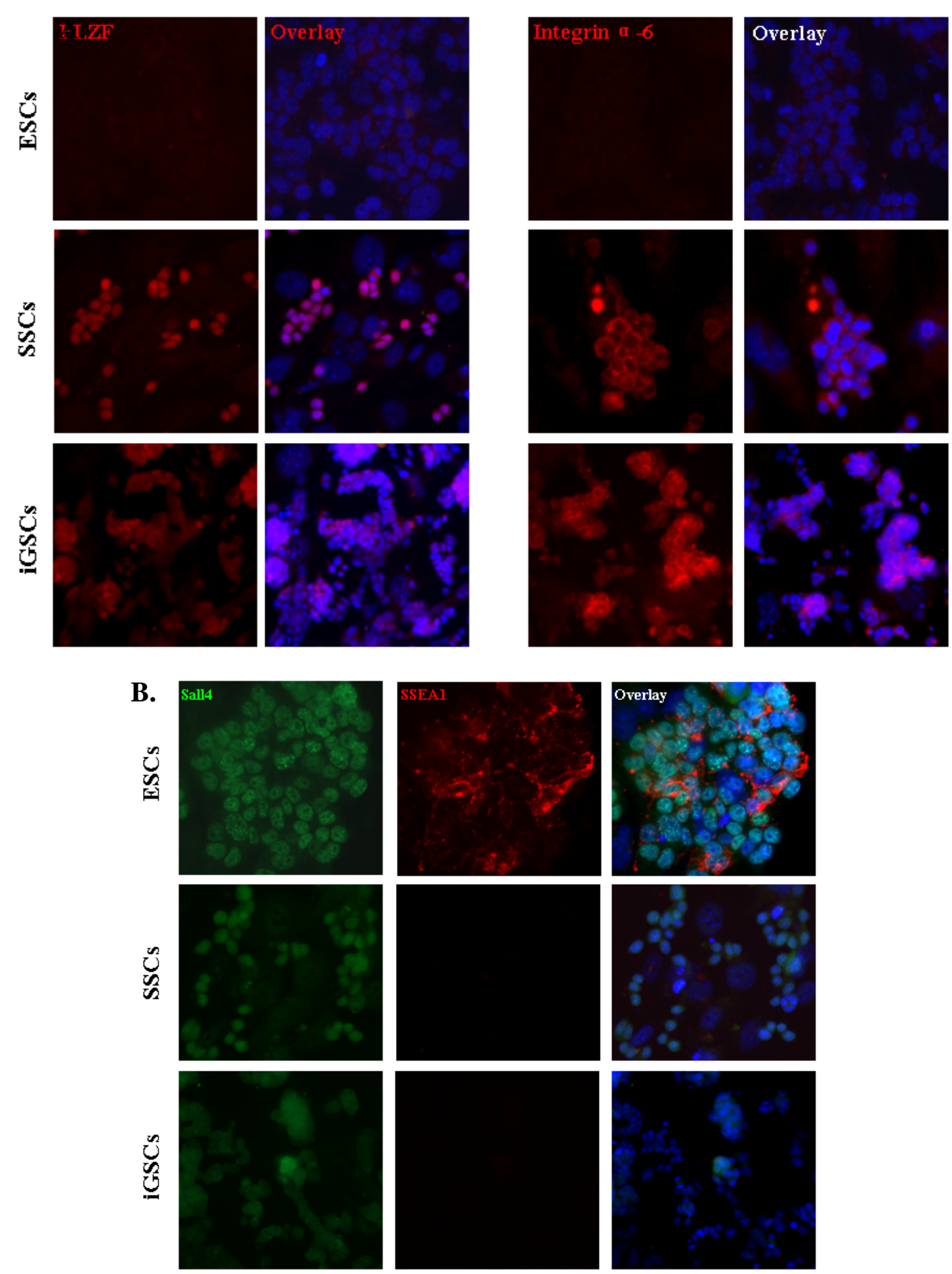

Figure 4.4. Characterization of iGSCs by immunocytochemistry. Immunocytochemical analysis for Plzf and integrin $\alpha-6$ (A) Sall4 and SSEA1 (B) in ESCs, SSCs and iGSCs. 


\subsection{Dppa3 binds to the IG-DMR of the Dlk1-Dio3 imprinting cluster and prevents its imprinting loss during iPS cell generation}

In the past few years, a variety of reprogramming methods have been established to generate iPS cells from somatic cells. Several standards for the evaluation of iPSCs pluripotency and their functional equivalence to ESCs have been accepted including molecular tests and in vivo developmental analysis. Among all these criteria, germ-line transmission was proposed to be one of the most stringent assessments reflecting the authenticity of iPS cells. Recently, two independent studies reported that the majority of mouse iPS cell lines show abnormal imprinting at the Dlkl-Dio3 locus and this leads to the loss of germline transmission of chimeras (Stadtfeld et al., 2010, Liu et al., 2010). The expression of Gtl2, a non-coding RNA of the Dlkl-Dio3 cluster, is being regarded as a bona fide marker for assessment of authentic pluripotent cell lines and thereby the germ line transmission competency.

Genomic imprinting is an epigenetic process of gene regulation during gametogenesis in mammals. Imprinted genes are characterized by differential DNA methylation and monoallelic expression according to the parent of origin-dependent manner (Spahn and Barlow, 2003). Imprinting control regions (ICRs) mediates the expression pattern of imprinted genes. Interestingly, many of the imprinted genes are grouped in domains, and the expression is regulated by cis-acting control elements which can repress multiple imprinted genes of the same domain. One such particular imprinting domain is at the Dlk1-Dio3 locus (delta-like 1 homolog-type III iodothyronine deiodinase) which is located on distal mouse chromosome $12(12 \mathrm{qF} 1)$ and human chromosome $14 \mathrm{q} 32$. Abnormal imprinting pattern at the Dlk1-Dio3 locus in humans is known to disrupt the function of the genes located in this locus and leads to a spectrum of disorders (table 4.1). 


\begin{tabular}{|c|c|c|c|c|c|c|}
\hline & matUPD14 & $\begin{array}{l}\text { Paternal deletion } \\
\text { of Dlk1-Gt/2 }\end{array}$ & $\begin{array}{l}\text { Paternal deletion } \\
\text { of Wars25- } \\
\text { snoRNAs }\end{array}$ & patUPD14 & $\begin{array}{l}\text { Maternal deletion of } \\
\text { Dlk1-Gt/2 }\end{array}$ & $\begin{array}{l}\text { Maternal deletion of } \\
\text { Wars25-snoRNAs }\end{array}$ \\
\hline $\begin{array}{l}\text { Predicted gene } \\
\text { dosage }^{\text {b }}\end{array}$ & $\begin{array}{l}\text { DLK1: 0x } \\
\text { ncRNAs: } 2 \mathrm{x} \\
\text { RTL1: 0x }\end{array}$ & $\begin{array}{l}D L K 1: 0 \mathrm{x} \\
\text { ncRNAs: 1x } \\
\text { RTL1: } 1 \mathrm{x}\end{array}$ & $\begin{array}{l}\text { DLK1: 0x } \\
\text { ncRNAs: } 1 \mathrm{x} \\
\text { RTL1: 0x }\end{array}$ & $\begin{array}{l}\text { DLK1: } 2 \mathrm{x} \\
\text { ncRNAs: } 0 \mathrm{x} \\
\text { RTL1: } 4 \mathrm{x}\end{array}$ & $\begin{array}{l}\text { DLK1: } 1 \mathrm{x} \\
\text { ncRNAs: } 0 \mathrm{x} \\
\text { RTL1: } 4 \mathrm{x}\end{array}$ & $\begin{array}{l}\text { DLK1: } 1 \mathrm{x} \\
\text { ncRNAs: } 0 \mathrm{x} \\
\text { RTL1: } 2 \mathrm{x}\end{array}$ \\
\hline $\begin{array}{l}\text { Facial } \\
\text { appearance }\end{array}$ & $\begin{array}{l}\text { High forehead, } \\
\text { short philtrum, } \\
\text { downturned } \\
\text { mouth }\end{array}$ & $\begin{array}{l}\text { Facial } \\
\text { dysmorphism } \\
\text { similar to } \\
\text { matUPD14 }\end{array}$ & $\begin{array}{l}\text { Facial } \\
\text { dysmorphism } \\
\text { similar to } \\
\text { matUPD14 }\end{array}$ & $\begin{array}{l}\text { Face-hairy forehead, } \\
\text { blepharophimosis, } \\
\text { micrognathia and } \\
\text { prominent philtrum }\end{array}$ & $\begin{array}{l}\text { Facial dysmorphism } \\
\text { as in patUPD14 }\end{array}$ & $\begin{array}{l}\text { Facial dysmorphism } \\
\text { similar to (but milder } \\
\text { than) patUPD14 }\end{array}$ \\
\hline Growth & $\begin{array}{l}\text { Pre- and postnatal } \\
\text { growth } \\
\text { retardation }\end{array}$ & $\begin{array}{l}\text { Pre- and } \\
\text { postnatal growth } \\
\text { retardation }\end{array}$ & $\begin{array}{l}\text { Pre- and } \\
\text { postnatal growth } \\
\text { retardation }\end{array}$ & $\begin{array}{l}\text { Normal prenatal growth; }{ }^{\mathrm{C}} \\
\text { severe postnatal growth } \\
\text { retardation }\end{array}$ & $\begin{array}{l}\text { Normal prenatal } \\
\text { growth; }{ }^{c} \text { postnatal } \\
\text { growth retardation }\end{array}$ & $\begin{array}{l}\text { Normal prenatal } \\
\text { growth }^{\mathrm{c}}\end{array}$ \\
\hline Motor system & $\begin{array}{l}\text { Joint laxity; } \\
\text { muscle hypotonia; } \\
\text { Motor delay }\end{array}$ & Motor delay & Motor delay & $\begin{array}{l}\text { Developmental delay; joint } \\
\text { contractures; feeding } \\
\text { difficulties }\end{array}$ & $\begin{array}{l}\text { Developmental } \\
\text { delay; joint } \\
\text { contractures; } \\
\text { feeding difficulties }\end{array}$ & $\begin{array}{l}\text { Developmental delay; } \\
\text { feeding difficulties }\end{array}$ \\
\hline Skeletum & $\begin{array}{l}\text { Reduced height } \\
\text { (by accelerated } \\
\text { skeletal } \\
\text { maturation); } \\
\text { dysmorphic face, } \\
\text { hands and feet }\end{array}$ & $\begin{array}{l}\text { Reduced height; } \\
\text { dysmorphic } \\
\text { features of face, } \\
\text { hands and feet }\end{array}$ & $\begin{array}{l}\text { Reduced height; } \\
\text { dysmorphic } \\
\text { features of face, } \\
\text { hands and feet }\end{array}$ & $\begin{array}{l}\text { Small and typical bell- } \\
\text { shaped thorax with 'coat- } \\
\text { hanger' appearance of the } \\
\text { ribs }\end{array}$ & $\begin{array}{l}\text { Small and typical } \\
\text { bell-shaped thorax } \\
\text { with 'coat-hanger' } \\
\text { appearance of the } \\
\text { ribs }\end{array}$ & $\begin{array}{l}\text { Small and typical } \\
\text { bell-shaped thorax } \\
\text { with 'coat-hanger' } \\
\text { appearance of the } \\
\text { ribs }\end{array}$ \\
\hline Placenta & nd & nd & nd & Placentomegaly & Placentomegaly & Placentomegaly \\
\hline $\begin{array}{l}\text { Neurological } \\
\text { processes }\end{array}$ & $\begin{array}{l}\text { Normal/mild } \\
\text { mental retardation }\end{array}$ & $\begin{array}{l}\text { No mental } \\
\text { retardation }\end{array}$ & $\begin{array}{l}\text { No mental } \\
\text { retardation }\end{array}$ & $\begin{array}{l}\text { Moderate to severe mental } \\
\text { retardation }\end{array}$ & ND & ND \\
\hline $\begin{array}{l}\text { Specific } \\
\text { characteristics }\end{array}$ & $\begin{array}{l}\text { Early puberty, } \\
\text { recurrent otitis } \\
\text { media; occasional } \\
\text { obesity }\end{array}$ & $\begin{array}{l}\text { Early puberty, } \\
\text { occasional } \\
\text { obesity }\end{array}$ & No early puberty & $\begin{array}{l}\text { Complicated pregnancies } \\
\text { with polyhydramnios and } \\
\text { premature delivery; } \\
\text { abdominal wall defects }\end{array}$ & $\begin{array}{l}\text { Complicated } \\
\text { pregnancies with } \\
\text { polyhydramnios and } \\
\text { premature delivery; } \\
\text { abdominal wall } \\
\text { defects }\end{array}$ & $\begin{array}{l}\text { Complicated } \\
\text { pregnancies with } \\
\text { polyhydramnios and } \\
\text { premature delivery; } \\
\text { mild abdominal wall } \\
\text { defects }\end{array}$ \\
\hline
\end{tabular}

Table 4.1 Pathological anomalies in human harbouring genetic defects at the Dlk1-Dio3 locus. matUPD, maternal uniparental disomy; patUPD, paternal uniparental disomy; snoRNA, small nucleolar RNA (Table adapted from Rocha et al., 2008)

In our previous study (Xu et al., 2011), we observed that the GC marker genes are activated earlier than endogenous pluripotency marker genes during somatic cell reprogramming. These results promoted us to investigate the role of GC marker genes in establishment of authentic pluripotency. In the present study (Xu et al., 2012, manuscript under review), we show that all iPS cell clones generated in presence of Dppa3 show normal imprinting at the Dlk1-Dio3 imprinting cluster. At the molecular level, we show that Dppa3 counteracts the binding of Dnmt3a, a de novo DNA methyltransferase, to this region thereby probably preventing the aberrant DNA methylation at this region during reprogramming. In line with a recent report, which showed that vitamin $\mathrm{C}$ can abolish the imprinting defect during reprogramming (Stadtfeld et al., 2011), we also observed that vitamin C can prevent the loss of imprinting at the Dlkl-Dio3 locus. Moreover, we observed a positive correlation between vitamin $\mathrm{C}$ and Dppa3, where the addition of vitamin $\mathrm{C}$ dramatically activated the expression of Dppa3 during very early stages of reprogramming. This early activation of Dppa3 might be sufficient for the maintenance/establishment of imprinting at the Dlk1-Dio3 locus. Thus, our study establishes Dppa3 as a genetic factor responsible for proper maintenance of imprinting during reprogramming and for the establishment of authentic pluripotent stem cells. 


\subsubsection{Functional analysis of Dppa3 during somatic cell reprogramming by inducible transgenic mouse model}

To gain more insights into the function of Dppa3 in establishment of authentic pluripotency and to study in which stage of reprogramming Dppa3 is important. We took advantage of doxycyclin-inducible system where the expression of Dppa3 can be temporally controlled. Towards this end, we constructed pTre_Dppa3 expression vector by cloning mouse Dppa3 open reading frame into pTre 3G vector. An ectopic hemagglutinin (HA) protein tag was added to the C-terminus of Dppa3 in order to facilitate immunoprecipitation assays afterwards (Fig. 4.5 A). The pTre_Dppa3 expression construct was then used to generate a transgenic mouse line. At the same time, an additional transgenic mouse line carrying the transactivator was generated. Further, the double transgenic mouse line was generated by inter-breeding of the two transgenic lines and currently, we are on the way to produce embryonic fibroblast cells and to perform iPS cells generation as outlined (Fig. 4.6). Briefly, after OSKM transduction, the Dppa3 transgene expression will be induced at indicated time point of reprogramming using doxycycline. The reprogramming time course samples in which the Dppa3 was activated at different time points will be analysed for Gtl2 expression. Moreover, the genome wide transcriptome might be necessarily performed from different samples to address the question of boosting reprogramming efficiency.

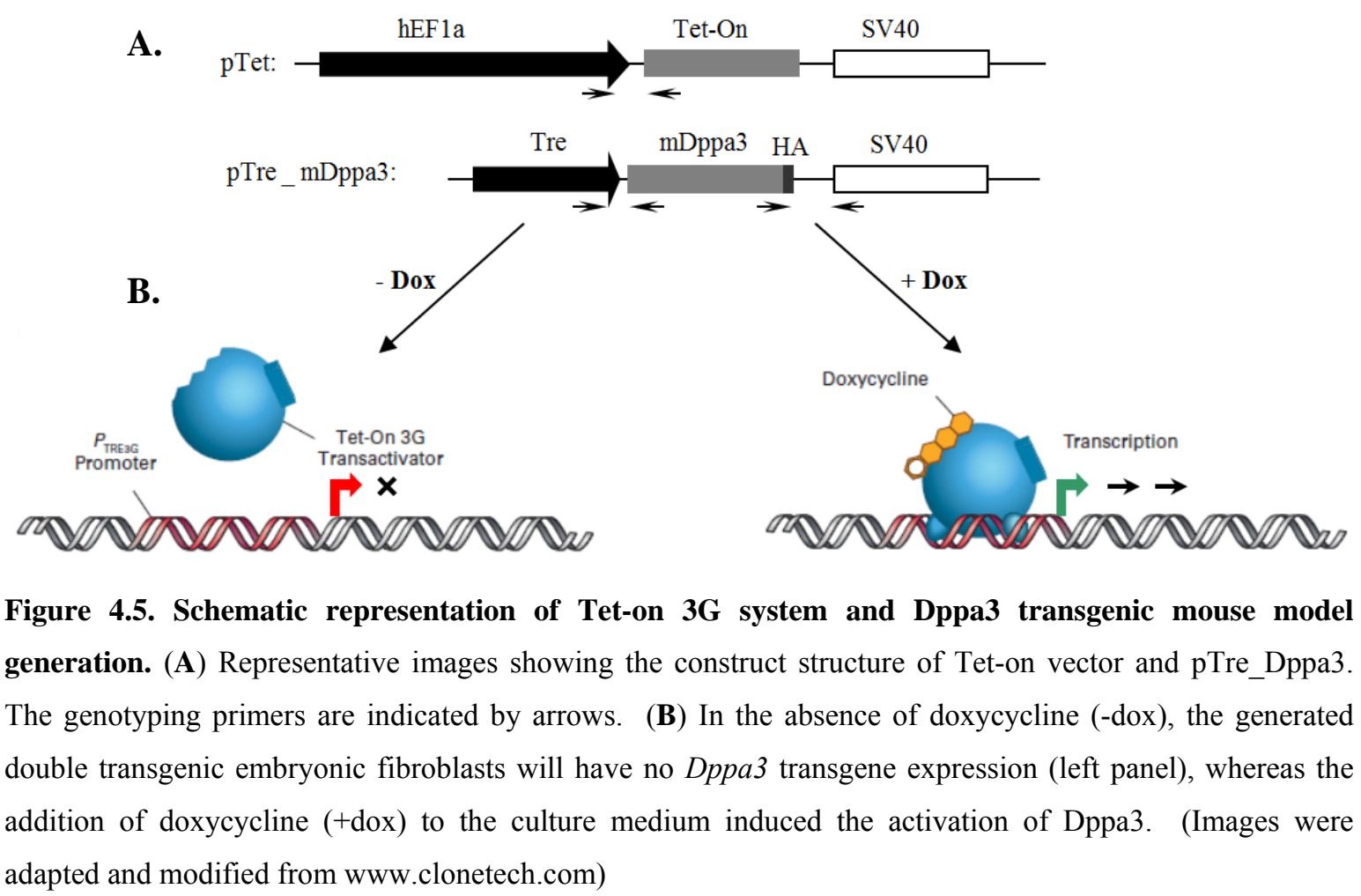




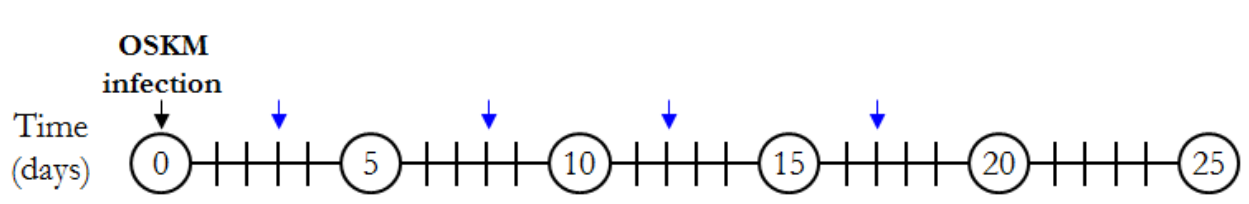

Figure 4.6. Schematic representation of induced Dppa3 expression during somatic cell reprogramming. The classic reprogramming factors (OSKM) will be delivered by retroviral infection. The expression of Dppa3 will be activated at indicated time points during the reprogramming by adding doxycycline (blue arrows) to the culture medium.

\subsection{Mouse Dazl and its novel splice variant function in translational repression of target mRNAs in mouse embryonic stem cells}

ESCs are regarded as a suitable model to study germ cell development in vitro and hold a great potential for reproductive medicine (Daley, 2007). In vitro generation of haploid gametes has been partly successful. The sperm-like cells derived from ESCs were able to fertilize oocytes by intracytoplasmic sperm injection (ICSI) (Hübner et al., 2003; Toyooka et al., 2003; Clark et al., 2004; Geijsen et al., 2004; Nayernia et al., 2006). A recent publication showed that the overexpression of Dazl, a PrM marker gene, in mouse ESCs leads to the generation of both motile tailed-sperm and oocytes (Yu et al., 2009). Subsequent functional studies showed that these artificial gametes could be used to produce early embryos until 8-cell stage ( $\mathrm{Yu}$ et al., 2009). This study further supports our assumption (Xu et al., 2011) that the expression of PrM marker genes is important for the germ cell lineage commitment of pluripotent stem cells.

Since 2004, artificial sperm has been derived in vitro in our group using several pluripotent cell types (Nayernia et al., 2004; Nayernia et al., 2006; Nolte et al., 2010). In general, our protocol is based on retinoic acid induced differentiation, analysis for the activation of the EGFP reporter from Stra8 promoter and subsequent separation of cells using fluorescence activated cell sorting (FACS). To overcome the transgene overexpression and reporter based approaches, which might cause the insertional mutagenesis, we attempted to use protein transduction strategy to generate germ cell-like cells. Previous studies have demonstrated that the conjugation of a short peptide such as HIV-Tat to a recombinant protein can facilitate the delivery of target protein into cells (Becker-Hapak et al., 2001; Albarran et al., 2005; Rayapureddi et al., 2010). To generate recombinant Dazl protein that can penetrate through the cell plasma membrane, we decided to fuse HIV-Tat polypeptide to the C terminus of Dazl (Fig. 4.7 A). The recombinant protein was expressed in E. coli and subsequently purified by using HisTrap purification 
system (Fig. 4.7 B). The Western blot analysis of the purified recombinant protein confirmed the successful purification of Dazl-Tat protein (Fig. 4.7 C). In order to test the function of Dazl-Tat recombinant protein in terms of cell permeability and stability, we treated immortal mouse fibroblast (NIH-3T3) cells. We observed that the Dazl-Tat recombinant protein easily penetrates cells (Fig. 4.7 D) and is stable inside of cells for at least 48 hours (data not shown).
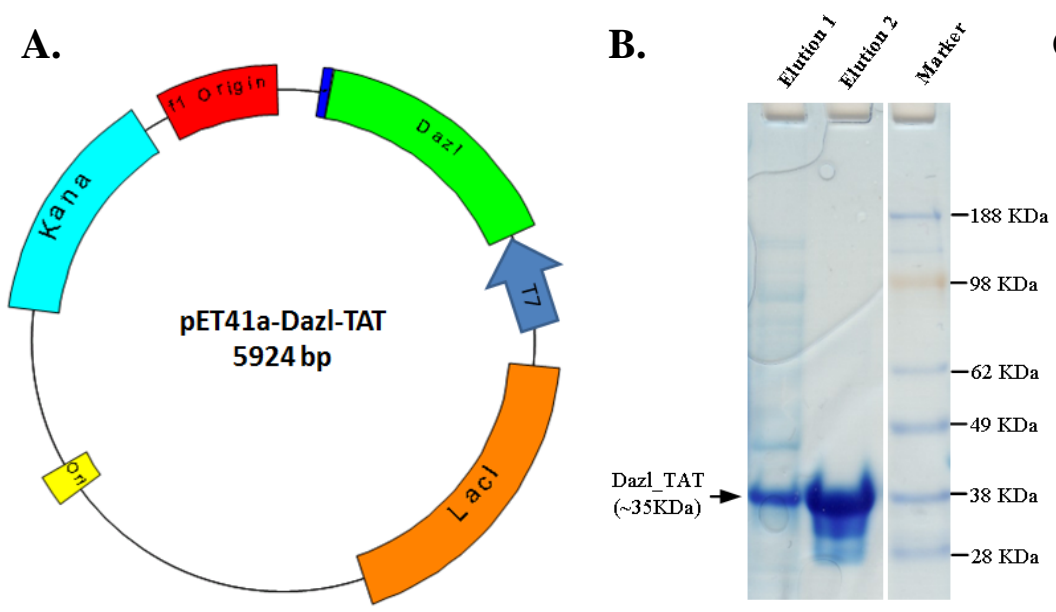

C.

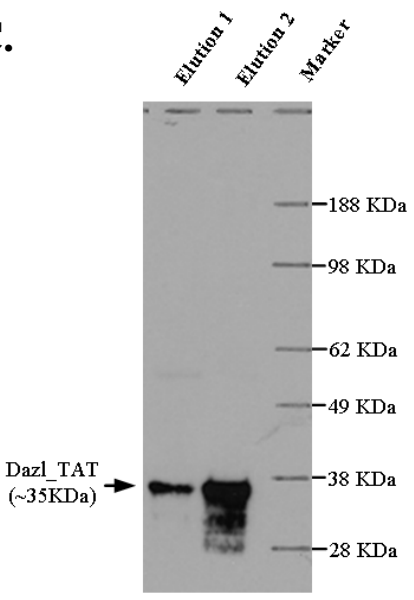

D.
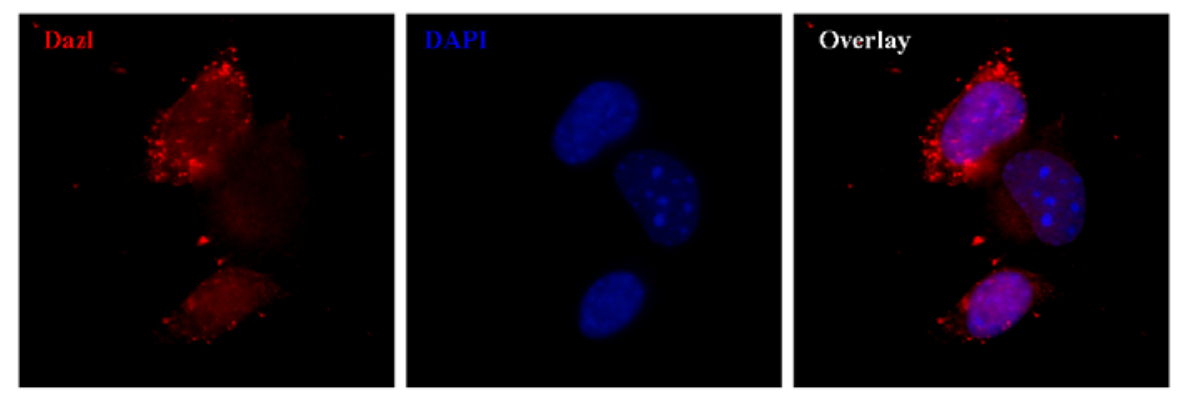

Figure 4.7. Dazl-Tat recombinant protein purification and functional test. (A) The representation of pET41a-Dazl-Tat construct, HIV-Tat polypeptide (blue) was tagged on to C terminus of mouse Dazl open reading frame. (B) Recombinant protein elution fraction Nr.1 and Nr.2 were analyzed on SDS-PAGE gel and staining with commassie blue. (C) The identity of recombinant protein elution fraction Nr.1 and Nr.2 were assessed by Western blot with Dazl antibody. (D) Dazl_Tat recombinant protein can penetrate the cell membrane and showed cytoplasmic locolization.

We then applied Dazl-Tat recombinant protein to ESCs in germ cell differentiation medium (Yu et al., 2009). However, we were unable to identify any sperm-like or oocytelike cells in our system. This could be due to the Dazl-Tat protein concentration levels which might not have been sufficient to trigger the germ cell differentiation. In contrast, the study by $\mathrm{Yu}$ et al (2009) employed lentiviral expression system, which gives the expression several orders of magnitudes than our protein delivery system. 
During the course of this study, we identified a novel splice variant of mouse Dazl (Dazl_48), which lacks exon 8 of Dazl. We then characterized the expression and localization pattern of this novel Dazl splice variant in pluripotent stem cells as well as in germ cells (Xu et al., 2012, manuscript under review). By comparison of protein domain structures, we found that a part of proline-rich (PR) region was lacking in the Dazl_ $\Delta 8$ isoform. It is known that PR regions mediate protein-protein interactions (Williamson, 1994). Therefore, it might be possible that Dazl_ $\Delta 8$ isoform is not able to interact with proteins such as Poly (A) binding family proteins and might lead to interaction with other unknown proteins thereby to regulate different targets. In contrast to the known function of translation stimulation in germ cells, we observed rather a translation repression function of Dazl in ESCs. In sub-cellular localization studies, we detected cytoplasmic localization of both isoforms, as it was reported for Dazl_FL protein (Lee et al., 2006; Kim et al., 2012). Interestingly, many cells showed stress granule (SG)-like as well as diffused cytoplasmic pattern for both Dazl isoforms. These results are in agreement with a recent report, which showed the Dazl-mediated SG formation and translation repression during induced stress conditions (Kim et al., 2012). In support of Dazl role in translation repression, through Coimmunoprecipitation (Co-IP) followed by mass spectrometry analysis, we could identify a heat shock protein 70 (HSP70), which is known to regulate the formation of TIA1mediated translational repression (Gilks et al., 2004), as a putative interaction partner of Dazl. These observations led us to suggest that under stress circumstances, formation of SGs is induced probably by HSP and Dazl complex to repress the translational event and to protect and store mRNAs. However, further studies are necessary to test this hypothesis and to show the mechanisms of translational repression function of Dazl in ESCs. 


\subsection{Future endeavors and perspectives}

In the present study, we elucidated the function of selected GC marker gene (Dppa3) and PrM marker gene ( $\mathrm{Dazl}$ ) in pluripotent stem cells. To gain deeper insights into the function of GC/PrM marker genes during reprogramming, it might also be necessary to establish iPS cell lines from GC/PrM overexpressed or knockedout fibroblasts to examine the gain or loss of pluripotency in comparison to wild type cells. The observation of GC marker genes expression in human fibroblast cells led us to hypothesize that mesenchymalto-epithelial transition (MET) will be activated early during reprogramming, as MET and GC marker genes are the responsive targets of the BMP signaling pathway. Therefore, there might be a crosstalk between the networks of MET genes and GC marker genes. Future studies aimed at investigation of MET in human fibroblast reprogramming might help to address the expression of GC marker genes in human fibroblast cells.

We observed an early activation of Dppa3 during somatic cell reprogramming and our further results implied its role in maintenance of the Dlk1-Dio3 imprinting. Although, the DNA binding ability of Dppa3 has been documented, its global genomic targets are still largely unknown. Therefore, the genome-wide screening for direct target based on ChIP followed by next generation sequencing techniques (ChIP-Seq) might help us to better understand Dppa3 role in epigenetic regulation of imprinting and gene expression. Dppa3 is a well-known maternally inherited epigenetic regulator and preferentially protects the maternal allele during zygotic DNA demethylation event. The study of Dppa3 binding to the Dlk1-Dio3 locus during reprogramming of fibroblasts isolated from F1 hybrid offspring of Mus musculus domesticus (C57BL6/J) and Mus musculus castaneus (CAST/EiJ) crosses might unravel the allele specific binding of Dppa3.

Although Dazl has been regarded as a post-transcriptional regulator, the repressive role of Dazl is still controversial and needs to be further studied. It might be necessary to perform electrophoretic mobility shift assays (EMSA) for studying the binding specificity of Dazl to its target RNA sequences. It was reported that the regulatory role of RNAbinding protein mainly accounts for interaction proteins. Therefore, identification of other interaction proteins of Dazl in ESCs might be useful to dissect the translational repression role of Dazl. 


\section{References}

Aflatoonian, B., Ruban, L., Jones, M., Aflatoonian, R., Fazeli, A., Moore, H. D., 2009. In vitro post-meiotic germ cell development from human embryonic stem cells. Hum Reprod. 24, 3150-9.

Albarran, B., To, R., Stayton, P. S., 2005. A TAT-streptavidin fusion protein directs uptake of biotinylated cargo into mammalian cells. Protein Eng Des Sel. 18, 14752.

Aravind, L., Koonin, E. V., 2000. SAP - a putative DNA-binding motif involved in chromosomal organization. Trends in biochemical sciences. 25, 112-4.

Bao, S., Leitch, H. G., Gillich, A., Nichols, J., Tang, F., Kim, S., Lee, C., Zwaka, T., Li, X., Surani, M. A., 2012. The germ cell determinant blimp1 is not required for derivation of pluripotent stem cells. Cell Stem Cell. 11, 110-7.

Becker-Hapak, M., McAllister, S. S., Dowdy, S. F., 2001. TAT-mediated protein transduction into mammalian cells. Methods. 24, 247-56.

Bilic, J., Izpisua Belmonte, J. C., 2012. Concise review: Induced pluripotent stem cells versus embryonic stem cells: close enough or yet too far apart? Stem Cells. 30, $33-41$.

Bortvin, A., Goodheart, M., Liao, M., Page, D. C., 2004. Dppa3 / Pgc7 / stella is a maternal factor and is not required for germ cell specification in mice. BMC Dev Biol. 4, 2.

Brons, I. G., Smithers, L. E., Trotter, M. W., Rugg-Gunn, P., Sun, B., Chuva de Sousa Lopes, S. M., Howlett, S. K., Clarkson, A., Ahrlund-Richter, L., Pedersen, R. A., Vallier, L., 2007. Derivation of pluripotent epiblast stem cells from mammalian embryos. Nature. 448, 191-5.

Bucay, N., Yebra, M., Cirulli, V., Afrikanova, I., Kaido, T., Hayek, A., Montgomery, A. M., 2009. A novel approach for the derivation of putative primordial germ cells and sertoli cells from human embryonic stem cells. Stem Cells. 27, 68-77.

Byrne, A., (2011-06-29), J., 2011. "The definition and etymology of the word pluripotency". eJournal of Cellular Biotechnology, 1;eP2.

Cauffman, G., Van de Velde, H., Liebaers, I., Van Steirteghem, A., 2005. DAZL expression in human oocytes, preimplantation embryos and embryonic stem cells. Mol Hum Reprod. 11, 405-11. 
Chan, Y. S., Yang, L., Ng, H. H., 2011. Transcriptional regulatory networks in embryonic stem cells. Prog Drug Res. 67, 239-52.

Chu, L. F., Surani, M. A., Jaenisch, R., Zwaka, T. P., 2011. Blimp1 expression predicts embryonic stem cell development in vitro. Curr Biol. 21, 1759-65.

Da Rocha, S. T., Edwards, C. A., Ito, M., Ogata, T., Ferguson-Smith, A. C., 2008. Genomic imprinting at the mammalian Dlk1-Dio3 domain. Trends Genet. 24, 306-16.

Daley, G. Q., 2007. Gametes from embryonic stem cells: a cup half empty or half full? Science. 316, 409-10.

De Felici, M., Dolci, S., Pesce, M., 1992. Cellular and molecular aspects of mouse primordial germ cell migration and proliferation in culture. Int J Dev Biol. 36, 205-13.

Eguizabal, C., Montserrat, N., Vassena, R., Barragan, M., Garreta, E., Garcia-Quevedo, L., Vidal, F., Giorgetti, A., Veiga, A., Izpisua Belmonte, J. C., 2011. Complete meiosis from human induced pluripotent stem cells. Stem Cells. 29, 1186-95.

Eguizabal, C., Shovlin, T. C., Durcova-Hills, G., Surani, A., McLaren, A., 2009. Generation of primordial germ cells from pluripotent stem cells. Differentiation. 78, 116-23.

Evans, M. J., Kaufman, M. H., 1981. Establishment in culture of pluripotential cells from mouse embryos. Nature. 292, 154-6.

Finch, B. W., Ephrussi, B., 1967. Retention of multiple developmental potentialities by cells of a mouse testicular teratocarcinoma during prolonged culture in vitro and their extinction upon hybridization with cells of permanent lines

Proc Natl Acad Sci U S A. 57, 615-21.

Fujiwara, Y., Komiya, T., Kawabata, H., Sato, M., Fujimoto, H., Furusawa, M., Noce, T., 1994. Isolation of a DEAD-family protein gene that encodes a murine homolog of Drosophila vasa and its specific expression in germ cell lineage. Proc Natl Acad Sci U S A. 91, 12258-62.

Gardner, R. L., Brook, F. A., 1997. Reflections on the biology of embryonic stem (ES) cells. Int J Dev Biol. 41, 235-43.

Geijsen, N., Horoschak, M., Kim, K., Gribnau, J., Eggan, K., Daley, G. Q., 2004. Derivation of embryonic germ cells and male gametes from embryonic stem cells. Nature. 427, 148-54. 
Gilks, N., Kedersha, N., Ayodele, M., Shen, L., Stoecklin, G., Dember, L. M., Anderson, P., 2004. Stress granule assembly is mediated by prion-like aggregation of TIA-1. Mol Biol Cell. 15, 5383-98.

Ginsburg, M., Snow, M. H., McLaren, A., 1990. Primordial germ cells in the mouse embryo during gastrulation. Development. 110, 521-8.

Gore, A., Li, Z., Fung, H. L., Young, J. E., Agarwal, S., Antosiewicz-Bourget, J., Canto, I., Giorgetti, A., Israel, M. A., Kiskinis, E., Lee, J. H., Loh, Y. H., Manos, P. D., Montserrat, N., Panopoulos, A. D., Ruiz, S., Wilbert, M. L., Yu, J., Kirkness, E. F., Izpisua Belmonte, J. C., Rossi, D. J., Thomson, J. A., Eggan, K., Daley, G. Q., Goldstein, L. S., Zhang, K., Somatic coding mutations in human induced pluripotent stem cells. Nature. 471, 63-7.

Guan, K., Nayernia, K., Maier, L. S., Wagner, S., Dressel, R., Lee, J. H., Nolte, J., Wolf, F., Li, M., Engel, W., Hasenfuss, G., 2006. Pluripotency of spermatogonial stem cells from adult mouse testis. Nature. 440, 1199-203.

Hanna, J. H., Saha, K., Jaenisch, R., 2010. Pluripotency and cellular reprogramming: facts, hypotheses, unresolved issues. Cell. 143, 508-25.

Haston, K. M., Tung, J. Y., Reijo Pera, R. A., 2009. Dazl functions in maintenance of pluripotency and genetic and epigenetic programs of differentiation in mouse primordial germ cells in vivo and in vitro. PLoS One. 4, e5654.

Hay, B., Jan, L. Y., Jan, Y. N., 1990. Localization of vasa, a component of Drosophila polar granules, in maternal-effect mutants that alter embryonic anteroposterior polarity. Development. 109, 425-33.

Hayashi, K., Ohta, H., Kurimoto, K., Aramaki, S., Saitou, M., 2011. Reconstitution of the mouse germ cell specification pathway in culture by pluripotent stem cells. Cell. $146,519-32$.

Hübner, K., Fuhrmann, G., Christenson, L. K., Kehler, J., Reinbold, R., De La Fuente, R., Wood, J., Strauss, J. F., 3rd, Boiani, M., Scholer, H. R., 2003. Derivation of oocytes from mouse embryonic stem cells. Science. 300, 1251-6.

Hussein, S. M., Batada, N. N., Vuoristo, S., Ching, R. W., Autio, R., Narva, E., Ng, S., Sourour, M., Hamalainen, R., Olsson, C., Lundin, K., Mikkola, M., Trokovic, R., Peitz, M., Brustle, O., Bazett-Jones, D. P., Alitalo, K., Lahesmaa, R., Nagy, A., Otonkoski, T., 2011. Copy number variation and selection during reprogramming to pluripotency. Nature. $471,58-62$. 
Hustin, J., Collette, J., Franchimont, P., 1987. Immunohistochemical demonstration of placental alkaline phosphatase in various states of testicular development and in germ cell tumours. Int J Androl. 10, 29-35.

Imamura, M., Aoi, T., Tokumasu, A., Mise, N., Abe, K., Yamanaka, S., Noce, T., 2010. Induction of primordial germ cells from mouse induced pluripotent stem cells derived from adult hepatocytes. Mol Reprod Dev. 77, 802-11.

Kahan, B. W., Ephrussi, B., 1970. Developmental potentialities of clonal in vitro cultures of mouse testicular teratoma. J Natl Cancer Inst. 44, 1015-36.

Kanatsu-Shinohara, M., Inoue, K., Lee, J., Yoshimoto, M., Ogonuki, N., Miki, H., Baba, S., Kato, T., Kazuki, Y., Toyokuni, S., Toyoshima, M., Niwa, O., Oshimura, M., Heike, T., Nakahata, T., Ishino, F., Ogura, A., Shinohara, T., 2004. Generation of pluripotent stem cells from neonatal mouse testis. Cell. 119, 1001-12.

Kim, B., Cooke, H. J., Rhee, K., 2012. DAZL is essential for stress granule formation implicated in germ cell survival upon heat stress. Development. 139, 568-78.

Kleinsmith, L. J., Pierce, G. B., Jr., 1964. Multipotentiality of Single Embryonal Carcinoma Cells. Cancer Res. 24, 1544-51.

Lacham-Kaplan, O., Chy, H., Trounson, A., 2006. Testicular cell conditioned medium supports differentiation of embryonic stem cells into ovarian structures containing oocytes. Stem Cells. 24, 266-73.

Lange, U. C., Adams, D. J., Lee, C., Barton, S., Schneider, R., Bradley, A., Surani, M. A., 2008. Normal germ line establishment in mice carrying a deletion of the Ifitm/Fragilis gene family cluster. Mol Cell Biol. 28, 4688-96.

Lange, U. C., Saitou, M., Western, P. S., Barton, S. C., Surani, M. A., 2003. The fragilis interferon-inducible gene family of transmembrane proteins is associated with germ cell specification in mice. BMC developmental biology. 3, 1.

Lawson, K. A., Dunn, N. R., Roelen, B. A., Zeinstra, L. M., Davis, A. M., Wright, C. V., Korving, J. P., Hogan, B. L., 1999. Bmp4 is required for the generation of primordial germ cells in the mouse embryo. Genes Dev. 13, 424-36.

Lee, K. H., Lee, S., Kim, B., Chang, S., Kim, S. W., Paick, J. S., Rhee, K., 2006. Dazl can bind to dynein motor complex and may play a role in transport of specific mRNAs. EMBO J. 25, 4263-70.

Lerou, P. H., Daley, G. Q., 2005. Therapeutic potential of embryonic stem cells. Blood Rev. 19, 321-31. 
Li, R., Liang, J., Ni, S., Zhou, T., Qing, X., Li, H., He, W., Chen, J., Li, F., Zhuang, Q., Qin, B., Xu, J., Li, W., Yang, J., Gan, Y., Qin, D., Feng, S., Song, H., Yang, D., Zhang, B., Zeng, L., Lai, L., Esteban, M. A., Pei, D., 2010. A mesenchymal-toepithelial transition initiates and is required for the nuclear reprogramming of mouse fibroblasts. Cell Stem Cell. 7, 51-63.

Liu, L., Luo, G. Z., Yang, W., Zhao, X., Zheng, Q., Lv, Z., Li, W., Wu, H. J., Wang, L., Wang, X. J., Zhou, Q., 2010. Activation of the imprinted Dlk1-Dio3 region correlates with pluripotency levels of mouse stem cells. The Journal of biological chemistry. 285, 19483-90.

Martin, G. R., 1981. Isolation of a pluripotent cell line from early mouse embryos cultured in medium conditioned by teratocarcinoma stem cells. Proc Natl Acad Sci U S A. 78, 7634-8.

Matsui, Y., 2009. The molecular mechanisms regulating germ cell development and potential. Journal of andrology. 31, 61-5.

Matsui, Y., Zsebo, K., Hogan, B. L., 1992. Derivation of pluripotential embryonic stem cells from murine primordial germ cells in culture. Cell. 70, 841-7.

McLaren, A., 1994. Introduction. In: Germline development.Ciba Foundation Symposium 182.Chichester: John Wiley\& Sons, 1994:1.

McLaren, A., 2001. Mammalian germ cells: birth, sex, and immortality. Cell Struct Funct. 26, 119-22.

Mise, N., Fuchikami, T., Sugimoto, M., Kobayakawa, S., Ike, F., Ogawa, T., Tada, T., Kanaya, S., Noce, T., Abe, K., 2008. Differences and similarities in the developmental status of embryo-derived stem cells and primordial germ cells revealed by global expression profiling. Genes Cells. 13, 863-77.

Nayernia, K., Li, M., Jaroszynski, L., Khusainov, R., Wulf, G., Schwandt, I., Korabiowska, M., Michelmann, H. W., Meinhardt, A., Engel, W., 2004. Stem cell based therapeutical approach of male infertility by teratocarcinoma derived germ cells. Hum Mol Genet. 13, 1451-60.

Nayernia, K., Nolte, J., Michelmann, H. W., Lee, J. H., Rathsack, K., Drusenheimer, N., Dev, A., Wulf, G., Ehrmann, I. E., Elliott, D. J., Okpanyi, V., Zechner, U., Haaf, T., Meinhardt, A., Engel, W., 2006. In vitro-differentiated embryonic stem cells give rise to male gametes that can generate offspring mice. Dev Cell. 11, 125-32.

Nichols, J., Silva, J., Roode, M., Smith, A., 2009. Suppression of Erk signalling promotes ground state pluripotency in the mouse embryo. Development. 136, 3215-22. 
Nichols, J., Smith, A., 2009. Naive and primed pluripotent states. Cell Stem Cell. 4, 48792.

Nichols, J., Smith, A., 2011. The origin and identity of embryonic stem cells. Development. 138, 3-8.

Nishikawa, S., Goldstein, R. A., Nierras, C. R., 2008. The promise of human induced pluripotent stem cells for research and therapy. Nature reviews. Molecular cell biology. 9, 725-9.

Nolte, J., Michelmann, H. W., Wolf, M., Wulf, G., Nayernia, K., Meinhardt, A., Zechner, U., Engel, W., 2010. PSCDGs of mouse multipotent adult germline stem cells can enter and progress through meiosis to form haploid male germ cells in vitro. Differentiation. 80, 184-94.

Ohinata, Y., Payer, B., O'Carroll, D., Ancelin, K., Ono, Y., Sano, M., Barton, S. C., Obukhanych, T., Nussenzweig, M., Tarakhovsky, A., Saitou, M., Surani, M. A., 2005. Blimp1 is a critical determinant of the germ cell lineage in mice. Nature. 436, 207-13.

Panula, S., Medrano, J. V., Kee, K., Bergstrom, R., Nguyen, H. N., Byers, B., Wilson, K. D., Wu, J. C., Simon, C., Hovatta, O., Reijo Pera, R. A., 2011. Human germ cell differentiation from fetal- and adult-derived induced pluripotent stem cells. Hum Mol Genet. 20, 752-62.

Park, T. S., Galic, Z., Conway, A. E., Lindgren, A., van Handel, B. J., Magnusson, M., Richter, L., Teitell, M. A., Mikkola, H. K., Lowry, W. E., Plath, K., Clark, A. T., 2009. Derivation of primordial germ cells from human embryonic and induced pluripotent stem cells is significantly improved by coculture with human fetal gonadal cells. Stem Cells. 27, 783-95.

Payer, B., Saitou, M., Barton, S. C., Thresher, R., Dixon, J. P., Zahn, D., Colledge, W. H., Carlton, M. B., Nakano, T., Surani, M. A., 2003. Stella is a maternal effect gene required for normal early development in mice. Curr Biol. 13, 2110-7.

Peng, J. X., Xie, J. L., Zhou, L., Hong, Y. H., Gui, J. F., 2009. Evolutionary conservation of Dazl genomic organization and its continuous and dynamic distribution throughout germline development in gynogenetic gibel carp. J Exp Zool B Mol Dev Evol. 312, 855-71.

Qing, T., Shi, Y., Qin, H., Ye, X., Wei, W., Liu, H., Ding, M., Deng, H., 2007. Induction of oocyte-like cells from mouse embryonic stem cells by co-culture with ovarian granulosa cells. Differentiation. 75, 902-11. 
Rayapureddi, J. P., Tomamichel, W. J., Walton, S. T., Payne, R. M., 2010. TAT fusion protein transduction into isolated mitochondria is accelerated by sodium channel inhibitors. Biochemistry. 49, 9470-9.

Reijo, R. A., Dorfman, D. M., Slee, R., Renshaw, A. A., Loughlin, K. R., Cooke, H., Page, D. C., 2000. DAZ family proteins exist throughout male germ cell development and transit from nucleus to cytoplasm at meiosis in humans and mice. Biol Reprod. 63, 1490-6.

Ren, B., Chee, K. J., Kim, T. H., Maniatis, T., 1999. PRDI-BF1/Blimp-1 repression is mediated by corepressors of the Groucho family of proteins. Genes Dev. 13, 12537.

Resnick, J. L., Bixler, L. S., Cheng, L., Donovan, P. J., 1992. Long-term proliferation of mouse primordial germ cells in culture. Nature. 359, 550-1.

Ruggiu, M., Speed, R., Taggart, M., McKay, S. J., Kilanowski, F., Saunders, P., Dorin, J., Cooke, H. J., 1997. The mouse Dazla gene encodes a cytoplasmic protein essential for gametogenesis. Nature. 389, 73-7.

Saiti, D., Lacham-Kaplan, O., 2007. Mouse Germ Cell Development in-vivo and in-vitro. Biomark Insights. 2, 241-52.

Saitou, M., Barton, S. C., Surani, M. A., 2002. A molecular programme for the specification of germ cell fate in mice. Nature. 418, 293-300.

Saitou, M., Yamaji, M., 2009. Germ cell specification in mice: signaling, transcription regulation, and epigenetic consequences. Reproduction. 139, 931-42.

Samavarchi-Tehrani, P., Golipour, A., David, L., Sung, H. K., Beyer, T. A., Datti, A., Woltjen, K., Nagy, A., Wrana, J. L., 2010. Functional genomics reveals a BMPdriven mesenchymal-to-epithelial transition in the initiation of somatic cell reprogramming. Cell Stem Cell. 7, 64-77.

Sato, M., Kimura, T., Kurokawa, K., Fujita, Y., Abe, K., Masuhara, M., Yasunaga, T., Ryo, A., Yamamoto, M., Nakano, T., 2002. Identification of PGC7, a new gene expressed specifically in preimplantation embryos and germ cells. Mech Dev. 113, 91-4.

Schlegel, P. N., 2009. Evaluation of male infertility. Minerva Ginecol. 61, 261-83.

Smith, K. P., Luong, M. X., Stein, G. S., 2009. Pluripotency: toward a gold standard for human ES and iPS cells. J Cell Physiol. 220, 21-9.

Spahn, L., Barlow, D. P., 2003. An ICE pattern crystallizes. Nature genetics. 35, 11-2. 
Stadtfeld, M., Apostolou, E., Akutsu, H., Fukuda, A., Follett, P., Natesan, S., Kono, T., Shioda, T., Hochedlinger, K., 2010. Aberrant silencing of imprinted genes on chromosome 12qF1 in mouse induced pluripotent stem cells. Nature. 465, 175-81.

Takahashi, K., Tanabe, K., Ohnuki, M., Narita, M., Ichisaka, T., Tomoda, K., Yamanaka, S., 2007. Induction of pluripotent stem cells from adult human fibroblasts by defined factors. Cell. 131, 861-72.

Takahashi, K., Yamanaka, S., 2006. Induction of pluripotent stem cells from mouse embryonic and adult fibroblast cultures by defined factors. Cell. 126, 663-76.

Tam, P. P., Snow, M. H., 1981. Proliferation and migration of primordial germ cells during compensatory growth in mouse embryos. J Embryol Exp Morphol. 64, $133-47$.

Tanaka, S. S., Toyooka, Y., Akasu, R., Katoh-Fukui, Y., Nakahara, Y., Suzuki, R., Yokoyama, M., Noce, T., 2000. The mouse homolog of Drosophila Vasa is required for the development of male germ cells. Genes Dev. 14, 841-53.

Tanaka, S. S., Yamaguchi, Y. L., Tsoi, B., Lickert, H., Tam, P. P., 2005. IFITM/Mil/fragilis family proteins IFITM1 and IFITM3 play distinct roles in mouse primordial germ cell homing and repulsion. Dev Cell. 9, 745-56.

Tedesco, M., La Sala, G., Barbagallo, F., De Felici, M., Farini, D., 2009. STRA8 shuttles between nucleus and cytoplasm and displays transcriptional activity. J Biol Chem. 284, 35781-93.

Tesar, P. J., Chenoweth, J. G., Brook, F. A., Davies, T. J., Evans, E. P., Mack, D. L., Gardner, R. L., McKay, R. D., 2007. New cell lines from mouse epiblast share defining features with human embryonic stem cells. Nature. 448, 196-9.

Tilgner, K., Atkinson, S. P., Golebiewska, A., Stojkovic, M., Lako, M., Armstrong, L., 2008. Isolation of primordial germ cells from differentiating human embryonic stem cells. Stem Cells. 26, 3075-85.

Toyooka, Y., Tsunekawa, N., Akasu, R., Noce, T., 2003. Embryonic stem cells can form germ cells in vitro. Proceedings of the National Academy of Sciences of the United States of America. 100, 11457-62.

Toyooka, Y., Tsunekawa, N., Takahashi, Y., Matsui, Y., Satoh, M., Noce, T., 2000. Expression and intracellular localization of mouse Vasa-homologue protein during germ cell development. Mechanisms of development. 93, 139-49. 
Tsui, S., Dai, T., Roettger, S., Schempp, W., Salido, E. C., Yen, P. H., 2000. Identification of two novel proteins that interact with germ-cell-specific RNAbinding proteins DAZ and DAZL1. Genomics. 65, 266-73.

Vincent, S. D., Dunn, N. R., Sciammas, R., Shapiro-Shalef, M., Davis, M. M., Calame, K., Bikoff, E. K., Robertson, E. J., 2005. The zinc finger transcriptional repressor Blimp1/Prdm1 is dispensable for early axis formation but is required for specification of primordial germ cells in the mouse. Development. 132, 1315-25.

West, F. D., Machacek, D. W., Boyd, N. L., Pandiyan, K., Robbins, K. R., Stice, S. L., 2008. Enrichment and differentiation of human germ-like cells mediated by feeder cells and basic fibroblast growth factor signaling. Stem Cells. 26, 2768-76.

Williamson, M. P., 1994. The structure and function of proline-rich regions in proteins. Biochem J. 297 ( Pt 2), 249-60.

Xu, X., Pantakani, D. V., Luhrig, S., Tan, X., Khromov, T., Nolte, J., Dressel, R., Zechner, U., Engel, W., 2011. Stage-specific germ-cell marker genes are expressed in all mouse pluripotent cell types and emerge early during induced pluripotency. PLoS One. 6, e22413.

Yang, S., Bo, J., Hu, H., Guo, X., Tian, R., Sun, C., Zhu, Y., Li, P., Liu, P., Zou, S., Huang, Y., Li, Z., 2012. Derivation of male germ cells from induced pluripotent stem cells in vitro and in reconstituted seminiferous tubules. Cell Prolif. 45, 91100.

Yu, J., Angelin-Duclos, C., Greenwood, J., Liao, J., Calame, K., 2000. Transcriptional repression by blimp-1 (PRDI-BF1) involves recruitment of histone deacetylase. Mol Cell Biol. 20, 2592-603.

Yu, Z., Ji, P., Cao, J., Zhu, S., Li, Y., Zheng, L., Chen, X., Feng, L., 2009. Dazl promotes germ cell differentiation from embryonic stem cells. J Mol Cell Biol. 1, 93-103.

Zhao, T., Zhang, Z. N., Rong, Z., Xu, Y., 2011. Immunogenicity of induced pluripotent stem cells. Nature. 474, 212-5.

Zhu, Y., Hu, H. L., Li, P., Yang, S., Zhang, W., Ding, H., Tian, R. H., Ning, Y., Zhang, L. L., Guo, X. Z., Shi, Z. P., Li, Z., He, Z., 2012. Generation of male germ cells from induced pluripotent stem cells (iPS cells): an in vitro and in vivo study. Asian J Androl. 14, 574-9.

Zwaka, T. P., Thomson, J. A., 2005. A germ cell origin of embryonic stem cells? Development. 132, 227-33. 


\section{Abbreviations}

${ }^{\circ} \mathrm{C}$

x g

$\mu-$

$\Delta 8$

Ac

Al

AP

AVE

Blimp1

BMP

bp

c-

cDNA

ChIP

ChIP-Seq

Co-IP

DAPI

Dazl

Dazl-FL

DE

Dio3

Dlk1

DN

DNA

Dox

dpc

dpp

Dppa3

DVE

ECCs

EGCs

EGFP degree Celsius

gravity

micro

Exon 8 deletion

Acetylation

Allantois

Alkaline Phosphatase

Anterior visceral endoderm

B- lymphocyte- induced maturation protein-1

Bone morphogenetic protein

base pair

centi

complementary DNA

Chromatin Immunoprecipitation

ChIP- Sequencing

Co-Imuunoprecipitation

4',6 -diamidino-2-phenylindole

Deleted in azoospermia-like

Dazl Full length

Distal endoderm

type III iodothyronine deiodinase

delta-like 1 homolog

Down regulation

Deoxyribonucleic acid

Doxycycline

days post coitum

days post partum

Developmental pluripotency-associated protein 3

Distal visceral endoderm

Embryonic carcinoma cells

Embryonic germ cells

Enhanced green fluorescent protein 
EM

EMSA

Epi

EpiSCs

ESCs

ExE

ExM

FACS

FB

FCS

FITC

GC

GSCs

$\mathrm{h}$

H3K27

H3K27me3

H3K4

H3K4me3

H3K9ac

H3K9me3

HA

hiPSCs

HRP

HSP70

ICC

ICM

ICRs

ICSI

Ifitm3

IG-DMR

iPSCs

k-

$\mathrm{kDa}$

$\mathrm{KO}$
Embryonic mesoderm

Electrophoretic mobility shift assay

Epiblast

Epiblast stem cells

Embryonic stem cells

Extra-embryonic ectoderm

Extra-embryonic mesoderm

Fluorescence activated cell sorting

Fibroblast

Fetal calf serum

Fluorescein Isothiocyanate

germ cell

Germline stem cells

hour

histone 3 lysine 27

trimethylation of histone 3 lysine 27

histone 3 lysine 4

trimethylation of histone 3 lysine 4

acetylation of histone 3 lysine 9

trimethylation of histone 3 lysine 9

hemagglutinin

human iPSCs

Horseradish peroxidase

Heat shock protein 70

Immunocytochemistry

Inner cell mass

Imprinting control regions

Intracytoplasmic sperm injection

Interferon-induced transmembrane protein 3

Intergenic-differentially methylated region

induced pluripotent stem cells

kilo

kilo Dalton

Knock-out 
1

LIF

$\mathrm{m}$

M

m-

maGSCs

MEFs

MET

mGSCs

$\min$

miRNA

mRNA

Mvh

OE

ORF

OSKM

PBS

PCR

PGC

$\mathrm{pH}$

PR

PrM

qRT-PCR

RA

RNA

RRM

RT-PCR

SCs

SDS

SDS-PAGE

SG

siRNA

SSCs

VE

liter

Leukemia inhibitory factor

meter

Molar

milli

multipotent adult germline stem cells

Mouse embryonic fibroblasts

Mesenchymal-to-epithelial transition

multipotent germline stem cells

minute

micro-RNA

messenger RNA

Mouse vasa homologue

Overexpression

Open reading frame

Oct4, Sox2, Klf4 and c-Myc

Phosphate buffered saline

Polymerase chain reaction

primordial germ cell

preponderance of hydrogen ions

proline-rich

pre- meiotic

quantitative RealTime-PCR

Retinoic acid

Ribonucleic acid

RNA recognition motif

Reverse transcriptase-PCR

Stem cells

Sodium dodecylsulfate

SDS-polyacrylamide gel electrophoresis

Stress granule

small interfering RNA

Spermatogonial stem cells

Visceral endoderm 


\section{Acknowledgments}

First of all, I wish to offer my sincerest gratitude to Prof. Dr. med. Dr. h. c. Wolfgang Engel, who has offered me such a invaluable opportunity to study in a prestigious stem cell research group, for his constant excellent guidance, inspiration, encouragement and for providing me with a harmonious atmosphere during my $\mathrm{PhD}$ study. It has been my great honor to study under his supervision and his personality and rigorous scholarship will motivate me for whole research life.

I sincerely thank all my thesis committee members, Prof. Dr. Sigrid HoyerFender, Prof. Dr. Detlef Doenecke, Prof. Dr. Michael Zeisberg, Prof. Dr. Michael Kessel, and Prof. Dr. Ahmed Mansouri for evaluation of my thesis and serving as my thesis committee.

Next, I would like express my deepest thank to my supervisor Dr. Krishna Pantakani for his continuous guidance, understanding, generosity, patience and helping me to develop my background in molecular biology and all techniques. I have been so lucky to have him as my group leader, friend and brother to company me throughout my $\mathrm{PhD}$ time. Without him this thesis would not have been completed or written.

My special thanks go to Dr. Jessica Nolte for her constitutive advice and discussions throughout my study.

I would like to thank Lukasz Smorag and Joanna Jakubiczka Smorag for their kindly help in my hardest time during PhD study. I am thankful to my colleagues Pawel, Tanja, Nadine, Sandra L, Janine, Sandra M, Gungee, Chiru, Belal, Mannar and all my institute members for their advice, discussions, help and providing an amicable working environment.

I would not have been here without them, so I would like to particularly thank Prof. Dr. Karim Nayernia and Christian Mueller, who recommended and introduced me to study at the Institute of Human Genetics and built a bridge from my Master's to PhD studies.

Last but not least, I want to express my deepest gratitude to my parents for supporting me in starting my scientific study, always put faith in me and helped to pass the hardest time during my study. I am grateful to my beloved wife, Xiaoying Tan who always backed me up, shared my ups and downs and gave me a family in Göttingen. 


\section{Curriculum Vitae}

\section{Personal details:}

Name:

Date of birth:

Place of birth:

Nationality:

Address:

E-Mail:

\section{Education:}

Sep. 1995 - Jul. 2001: $\quad$ Secondary School

Experimental Middle School, Tongliao, China

Sep.2001 - Jul.2005: $\quad$ Bachelors in Biology

Diploma thesis: "The study of cell apoptosis induced by potassium chromate"

College of Life Science, Dalian Nationalities University, China

Sep.2007 - Aug.2008: $\quad$ Masters in Medical and Molecular Biosciences (MRes) Master thesis: "The derivation of male meiotic germ line cells from mouse embryonic stem cells"

Medical School, Newcastle University,

Newcastle upon Tyne, U.K

Since Jun. 2009:
PhD thesis: "Stage-specific germ cell marker genes function in establishment and germ cell lineage commitment of pluripotent stem cells"

Institute of Human Genetics, Georg-August-University, Göttingen, Germany 


\section{List of Publications}

1. Xu X, Pantakani DV, Luhrig S, Tan X, Khromov T, Nolte J, Dressel R, Zechner U, Engel W. (2011) Stage-specific germ-cell marker genes are expressed in all mouse pluripotent cell types and emerge early during induced pluripotency. PLoS One 6(7): e22413.

\section{List of Manuscripts in Submission Stage}

1. Xu X, Nakamura T, Smorag L, Dressel R, Fitzner A, Linke M, Nolte J, Zechner U, Engel W, Pantakani DV. Dppa3 binds to the IG-DMR of the Dlk1-Dio3 imprinting cluster and prevents its imprinting loss during iPS cell generation. (Manuscript under review).

2. Xu X, Tan X, Lin Q, Schmidt B, Engel W, Pantakani DV. (2012) Dazl and its novel splice variant functions in translational repression of target mRNAs in mouse embryonic stem cells. (Manuscript under review).

3. Nyamsuren $\mathrm{G}^{*}, \mathbf{X u} \mathbf{X}^{*}$, Taka O, Doerfel L, Pantakanai DV, Engel W, Adham I. PELO functions in establishment of pluripotency and development of primitive endoderm through the activation of BMP-signaling pathway. (*Equal contribution, Manuscript under review).

4. Tan X, Xu X, Zechner U, Nolte J, Engel W, Pantakanai DV. Zfp819, a novel KRABzinc finger protein, interacts with KAP1 and functions in genomic integrity maintenance of mouse embryonic stem cells. (Manuscript under review). 